\title{
WestVirginiaUniversity
}

THE RESEARCH REPOSITORY @ WVU

Graduate Theses, Dissertations, and Problem Reports

2003

\section{AFAP-110 is a cSrc activator}

Joseph Myers Baisden

West Virginia University

Follow this and additional works at: https://researchrepository.wvu.edu/etd

\section{Recommended Citation}

Baisden, Joseph Myers, "AFAP-110 is a cSrc activator" (2003). Graduate Theses, Dissertations, and Problem Reports. 871.

https://researchrepository.wvu.edu/etd/871

This Dissertation is protected by copyright and/or related rights. It has been brought to you by the The Research Repository @ WVU with permission from the rights-holder(s). You are free to use this Dissertation in any way that is permitted by the copyright and related rights legislation that applies to your use. For other uses you must obtain permission from the rights-holder(s) directly, unless additional rights are indicated by a Creative Commons license in the record and/ or on the work itself. This Dissertation has been accepted for inclusion in WVU Graduate Theses, Dissertations, and Problem Reports collection by an authorized administrator of The Research Repository @ WVU.

For more information, please contact researchrepository@mail.wvu.edu. 


\title{
AFAP-110 is a cSrc activator
}

\author{
Joseph M. Baisden
}

Dissertation submitted to the School of Medicine at West Virginia University in partial fulfillment of the requirements for the degree of

\author{
Doctor of Philosophy \\ in \\ Microbiology and Immunology \\ Daniel C. Flynn, Ph.D, Chair \\ Thomas A. Elliott, Ph.D. \\ Stephen G. Graber, Ph.D. \\ Bing-Hua Jiang, Ph.D. \\ Mary J. Wimmer, Ph.D. \\ Department of Microbiology and Immunology \\ Morgantown, West Virginia \\ 2003
}

Keywords: Src, actin, cytoskeleton, PKC 
Abstract

AFAP-110 is a cSrc activator

Joseph M. Baisden

The cSrc oncogene directs the reorganization of the actin cytoskeleton in both normal and transformed cells. Mutation of protein binding domains (SH2 and SH3) from cSrc results in the deregulation of the kinase and the transformation of cells, while deletion of these domains blocks transformation by active forms of Src. This indicates that SH2/SH3 binding partners for cSrc may play a crucial role in the regulation of this kinase as well as the fulfillment of cSrc-directed functions. One SH2/SH3 binding partner for cSrc being investigated for its role in cSrc transformation is the actin filament-associated protein, AFAP-110. AFAP-110 represents a potential regulator of the actin cytoskeleton, as it is associated with actin structures and contains an intrinsic potential to direct the reorganization of the cytoskeleton in a manner which resembles Src transformation. In this work we addressed this potential for AFAP-110 to induce actin reorganization. Immunoblot and immunofluorescence analyses were used to investigate this potential. The results indicate that AFAP-110 binds actin directly and can induce cytoskeletal reorganizations in multiple cells types. Additionally, AFAP-110 can activate cSrc in an SH3 domain-dependent mechanism. This ability is revealed upon phosphorylation by PKC, which induces a conformational change in AFAP-110. Both SH3 interactions with cSrc and $\mathrm{PH}$ domain interactions with $\mathrm{PKC}$ are required for this result, as abrogation of either interaction blocks both the activation of cSrc and the reorganization by PKC. These results allow us to conclude that AFAP-110 can activate cSrc in response to cellular signals. 


\section{Dedication}

I dedicate this work to my wife, Jamie, and my children, Kailani and Matthew. 


\section{Acknowledgements}

I would like to thank the following:

my committee members for their help;

Dr. Daniel Flynn for guidance and support;

members of the Flynn lab;

members of the Landreth, Ericson, and Gibson labs;

Dr. Robert Craig, Director of the M.D./Ph.D. program;

my parents, E.J. and Judy;

and especially my family, Jamie, Kailani and Matthew. 


\section{CONTENTS}

PAGE

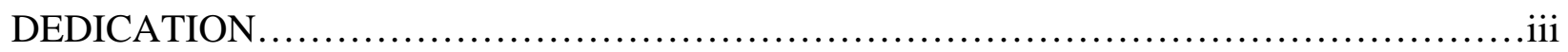

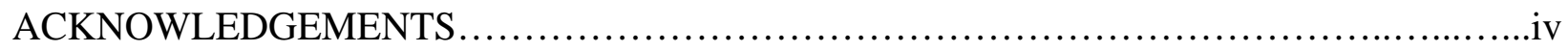

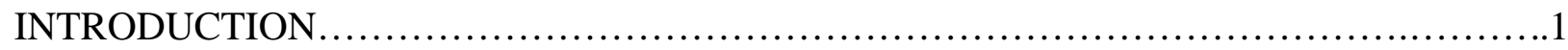

CHAPTER 1

AFAP-110 binds actin directly and has the intrinsic ability to induce cytoskeletal

rearrangements 31

CHAPTER 2

The intrinsic ability of AFAP-110 to alter actin filament integrity is linked with its ability to also activate cellular tyrosine kinases.

\section{CHAPTER 3}

AFAP-110 activates Src through SH3 interactions in response to PKC

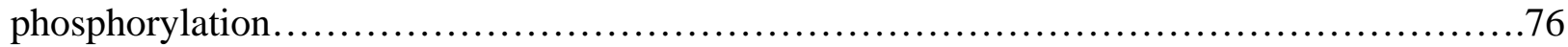

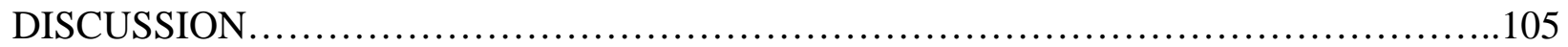

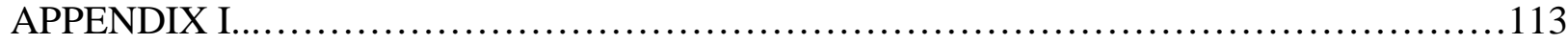

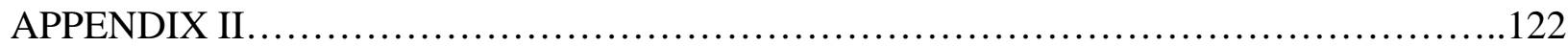

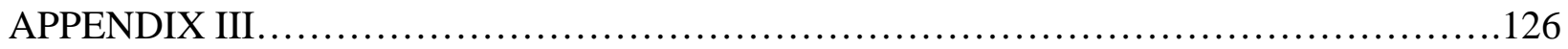

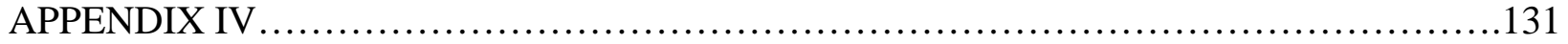

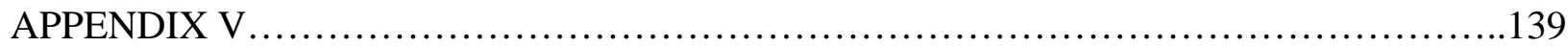

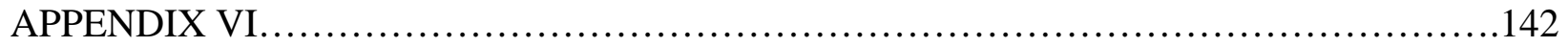

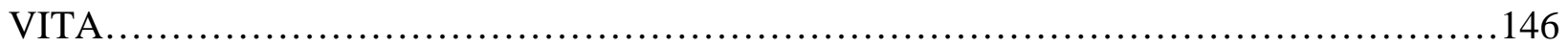




\section{Introduction}

AFAP-110 is a potential modulator of cytoskeletal rearrangements in response to cellular signals involving Src and/or PKC. 
The actin cytoskeleton is an important structural element of the cell which is involved in many cellular processes. This framework gives the cell strength and stability which contribute to the ultrastructure of organs as well as to other functions of cells. The cytoskeleton is not static in many cells as it must undergo reorganization in order for cells to move or divide. One signaling component studied for its ability to direct cytoskeletal rearrangements is the nonreceptor tyrosine kinase cSrc. cSrc is an enzyme which is tightly regulated in nontransformed cells but is often hyperactive in tumor cells, particularly those with increased capacity to metastasize. Much work has been done at determining the mechanism whereby cSrc directs cytoskeletal rearrangements and subsequent increased cell migration. One protein which has been studied for its involvement in this process is the actin filament associated protein $110 \mathrm{kDa}$, AFAP-110. The research presented here addresses potential mechanisms whereby AFAP-110 may mediate cSrc-directed cytoskeletal rearrangements.

\section{The actin cytoskeleton serves to both anchor cells and allow cells to migrate.}

The structural strength and stability of a cell is largely provided by the cytoskeleton. The cytoskeleton is comprised of several distinct structures, including intermediate filaments, microtubules and actin filaments. This network of structural proteins is mainly inside cells, but connections with extracellular matrix proteins provide a continuous framework on which the cytoskeleton is built. These connections also anchor the cells in place, in the case of non-motile cells. The actin filaments are the major cytoskeletal component in most cells, like the epithelial cells shown in Figure 1. These cells have been labeled to display the actin cytoskeleton, which appears as filaments within the cells which are networked to other cells through extracellular connections to maintain the integrity of the epithelial barrier. In cells which are required to move for function, the actin cytoskeleton is dynamically reorganized to provide the mechanical 


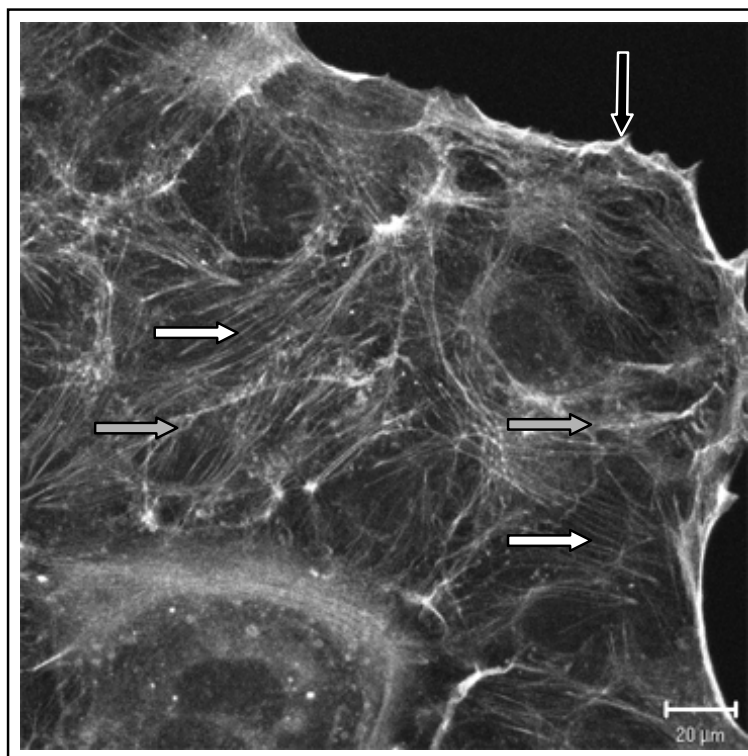

Figure 1 The actin cytoskeleton in epithelial cells (Caco-2) provides the structural framework inside the cells (white arrows) that connects with the cytoskeleton of other cells at cell-cell junctions (gray arrows). The cytoskeleton also extends from the edge of the epithelial sheet (black arrow) to direct the closure of the sheet, such as in wound healing or organ development. force necessary for this movement. This dynamic rearrangement is normally tightly controlled, and is induced via signaling pathways in response to extracellular clues which guide the cell's movement.

The actin cytoskeleton is dynamically reorganized in response to extracellular signals to direct cell motility.

Extracellular signals direct the normal growth and development of organs by directing the migration of cells to the organ and their further proliferation. These signals come from a plethora of sources, including immune/MHC receptors, adhesion receptors, receptor protein

tyrosine kinases (RPTKs), G protein-coupled receptors (GPCRs), cytokine receptors and GPIlinked receptors. Many of these receptors direct the reorganization of the actin cytoskeleton upon ligand binding to influence the cell to migrate in the direction of the signal. This migration toward a gradient is a common mechanism exploited by various cell types which migrate including cells of the immune system as well as cells in the developing organism.

The receptors which transduce extracellular signals are divided into at least 6 types, all of which appear to facilitate ctyoskeletal rearrangements. The immune/MHC receptors are a family of proteins comprised of multiple subunits which cooperate to bind an extracellular ligand and transduce a signal to the cytoplasm. This family includes $\mathrm{T}$ and $\mathrm{B}$ cell antigen receptors and $\mathrm{Fc}$ 
receptors which are necessary for immune responses (Hulett and Hogarth, 1994; Weiss and Littman, 1994). These immune responses require cytoskeletal rearrangements in order for proper functionality (Barois et al., 1998; Valitutti et al., 1995). The adhesion receptors consist of several distinct classes of proteins including the integrins, cadherins, selectins, and cellular adhesion molecules (CAMs) (Gumbiner, 1993; Tang and Honn, 1994). These proteins represent a wide variety of structurally distinct molecules which serve to provide adherence for the cell to the extracellular matrix (Giancotti and Ruoslahti, 1999). This adherence depends upon the integrity of the actin cytoskeleton, which is affected by the adhesion receptors in a signaldependent fashion. RPTKs are transmembrane proteins which bind soluble ligands like PDGF, EGF, FGF, CSF and a host of others (Kazlauskas, 1994). As their name suggests, these proteins contain an intrinsic kinase activity which they utilize to invoke various signaling pathways and induce cellular responses. Often this response includes cytoskeletal rearrangements. The GPCRs are the seven transmembrane-spanning proteins which are linked to heterotrimeric G protein complexes (van Biesen et al., 1996). These receptors recognize a variety of extracellular signaling molecules, including LPA, Thrombin, Angiotensin II, Bombesin, Bradykinin and Vasopressin, and induce cellular response via altering $\mathrm{G}$ protein complexes. These receptors can also induce cytoskeletal rearrangements. The next family of receptors are the transembrane cytokine receptors which are divided into two groups based on shared structural features. These receptors recognize many signaling molecules including interleukins 2-7, IL-11, IL-12, IL-15, GCSF, GM-CSF, TNF and erythropoeitin and induce effects in part through direct interactions with intracellular kinases. By activating these kinases, these receptors can induce cytoskeletal rearrangements. The GPI-coupled receptors are extracellular proteins which induce cytoplasmic signaling (Englund, 1993). Molecules recognized by this class include Thy-1, Ly-6, CD14, CD 
45, CD24, CD55 and CD59. These receptors are thought to interact with other transmembrane proteins, like integrins and LFA to transduce cellular signals which result in cytoskeletal rearrangements (Petty et al., 1997). As the engagement of each of these types of receptors results in cytoskeletal rearrangements, understanding the signals involved may shed light on a common mechanism and greatly support the importance of this mechanism in a wide range of signaling pathways.

\section{Extracellular signals that result in cytoskeletal reorganizations utilize cSrc.}

There is evidence that family members of each of the receptors listed above interact with and alter the activity of cSrc family members (Thomas and Brugge, 1997). Thus, cSrc may represent a component of a common mechanism employed by all of these receptors to direct cytoskeletal rearrangements. To understand the potential role of cSrc in these cytoskeletal reorganizations, it is helpful to also understand the regulation of cSrc kinase activity.

The cSrc oncogene is normally maintained in a repressed state and is activated to induce cytoskeletal reorganizations necessary to cell migration.

The cSrc oncogene is one of the most intensely studied proteins as it was the first oncogene characterized as well as the first tyrosine kinase identified (Brugge and Erikson, 1977; Collett et al., 1978; Levinson et al., 1978; Purchio et al., 1978). There are at least 10 proteins in the Src family of tyrosine kinases, which show varied expression in tissues. Src, Yes, Yrk and Fyn are ubiquitously expressed. Lyn, Hck, Fgr, Blk, and Lck are found in hematopoietic cells; Lyn and Lck are also found in brain. The Frk subfamily is found in epithelial cells. These expression patterns suggest Src and family members may be important to nearly all cell types. cSrc is maintained in an enzymatically repressed state through a mechanism thought to largely involve intramolecular interactions (Brown and Cooper, 1996). In the normal function of cells, 
these repressive interactions are relieved upon the receipt of specific signals, resulting in the activation of cSrc. This is thought to direct the reorganization of the actin cytoskeleton during cytokinesis and cell migration.

The intramolecular interactions which govern cSrc activity involve several of the domains of the molecule. Src family molecules have been artificially divided into domains which have been named SH1 through SH4. The SH4 domain is the amino-terminal $7-14$ residues of the molecule and includes at least a myristoylation signal and often an additional palmitoylation signal, which results in the insertion of this hydrophobic moiety into lipid bilayers, effectively anchoring the kinase at the cell membrane. Adjacent to this is the unique domain, the least conserved domain among Src family members. The function of this domain is largely unknown. Carboxy-terminal to the unique domain is the SH3 domain, which has affinity for proline-rich binding partners. SH3 domains are found in various signaling proteins outside of the Src family as well. Following the SH3 domain is the SH2 domain, which is involved in binding tyrosine-phosphorylated proteins. Like the SH3 domain, this type of domain is also found in non-Src signaling proteins. The SH1 domain is near the carboxy-terminus and is the catalytic portion of the molecule. This kinase portion is well conserved among tyrosine kinases. The SH1 domain is followed by a carboxy-terminal tail which plays a key role in the regulation of the molecule. This tail contains a tyrosine (residue 527 in chick, 530 in human) which is normally phosphorylated by cSrc kinase (CSK), enabling it to efficiently bind the SH2 domain of Src. This also brings the SH3 domain of Src in contact with a proline-rich linker region between the SH3 domain and the kinase domain. Together, these interactions result in a conformational change in the kinase domain which precludes the coordination of ATP, effectively inhibiting kinase activity. Src is thought to overcome this repression normally by the dephosphorylation of 
the carboxy-terminus by protein phosphatases. Several different phosphatases which may be activated in various cell types have been shown to activate Src by this mechanism, including PTP $\alpha$, PTP $\lambda$, RPTPE, PTP1B, SHP-1 and SHP-2 (Thomas and Brugge, 1997). Thus, the normal regulation of Src is thought to proceed through a well-studied mechanism.

Evidence exists for the activation of Src family kinases by other mechanisms which have an impact on the studies presented here. Originally, mutations in either the SH3 domain or the linker which it contacts within Src family kinases were shown to result in activation, indicating a necessary role for this interaction in the mechanism of repression (Gonfloni et al., 1997; Reynolds et al., 1992; Weil and Veillette, 1994; Wright et al., 1994). Other studies have supported this hypothesis. Hck has been shown to be activated by SH3 engagement. The HIV protein Nef, which can transform cells and may be involved in the propagation of HIV, is an SH3 binding partner for Hck (Moarefi et al., 1997). The high affinity binding between Nef and Hck result in the activation of Hck, presumably by relieving the conformational restraints on the kinase domain and allowing ATP coordination and incorporation into substrates. Collette et al. also showed that Nef proteins from HIV-2 and SIV demonstrate SH3 interactions with both Src and Fyn, indicating this type of activation may be relevant for other Src family members (Collette et al., 2000). Work with the Tip protein from Herpesvirus saimiri strain 484C supports this, as a similar activation effect on the Src family member Lck has been shown. SH3 engagement between these two proteins also results in the activation of Lck kinase activity (Hartley et al., 1999; Hartley et al., 2000). Furthermore, recent work has shown that the SH2 and SH3 domains act together in a "snap-lock" type mechanism to repress Src family kinase activity. Young et al. reported this result through a series of computer modeling and genetic experiments, showing that the linker between the $\mathrm{SH} 2$ and $\mathrm{SH} 3$ domains was important to 
repressing the kinase (Young et al., 2001). This result further supports the idea that simple SH3 engagement may allow for the efficient activation of the kinase. Thus, Src family kinases may be activated by mechanisms involving SH3 engagement.

The creation of knockout cSrc mice has shed light on the roles that ccSrc plays in the regulation of specialized actin structures. cSrc -/- mice display osteoclast dysfunction seen clinically as osteopetrosis, which is correlated with the inability of these cells to form lamellipodia (Boyce et al., 1992; Horne et al., 1992; Schwartzberg et al., 1997; Soriano et al., 1991). Lamellipodia are specialized actin structures which, along with filopodia, actin filaments, and focal adhesions, are thought to be involved in cell motility (Maidment, 1997). Kinase activity may not be required for this cSrc function, since expression of kinase-inactive Src rescues osteoclast function (Schwartzberg et al., 1997). An analogous situation has been shown in fibroblasts isolated from these knockout mice and implicates cSrc in the regulation of cell motility. These fibroblasts display delayed adhesion and spreading as well as decreased rates of motility, traits rescued by expression of kinase inactive Src (Hall et al., 1996; Kaplan et al., 1995). These functions may be regulated by protein-protein interactions, as the SH2 and SH3 domains of Src are required for rescue. Other studies have confirmed Src's involvement in cell motility directed by other signal transduction pathways. It was demonstrated that the expression of dominant negative Src inhibits the locomotion mediated by the RHAMM-receptor for hyaluron (Hall et al., 1996). Furthermore, cSrc has been implicated in the EGF-induced migration of a rat carcinoma cell line (Rodier et al., 1995) as well as endothelial cell migration induced by the inhibition of angiotensin II (Bell et al., 1992). Thus, cSrc plays a crucial role in cytoskeletal rearrangements which are necessary for cell migration in response to extracellular signals. 


\section{Deregulated activation of Src results in characteristic cytoskeletal reorganizations.}

The vSrc oncogene encoded by the RSV genome displays a loss of regulation of Src kinase activity and this constitutive activation results in the transformation of cells (Thomas and Brugge, 1997). This model of transformation has been used to gain understanding about the characteristics of cancerous transformation. Expression of a mutated form of cSrc in which the regulatory tyrosine has been mutated to phenylalanine $\left(\mathrm{Src}^{527 \mathrm{~F}}\right)$ also results in cell transformation. From these studies it was deduced that the hallmark of Src transformation is the reorganization of the actin cytoskeleton. This reorganization is characterized by the loss of actin filaments and the formation of structures sometimes termed rosettes, which appear as actin-rich punctate formations. These cells also display lamellipodia and membrane ruffles, both of which are involved in the movement of cells. Together, this change in phenotype is thought to indicate an increased ability to move. Figure 2 displays this phenotype, comparing non-transformed fibroblasts (panel A) with $\mathrm{Src}^{527 \mathrm{~F}}$-transformed fibroblasts (panel B).
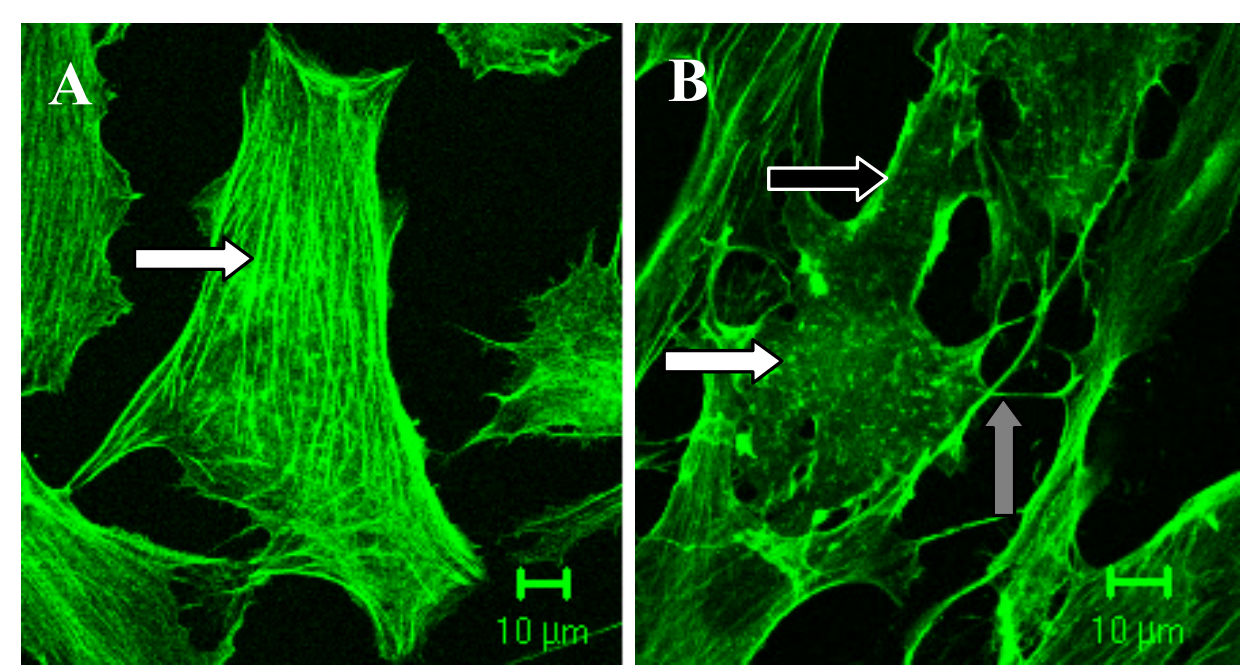

Figure 2 Quiescent fibroblasts display actin filaments (panel A, white arrows), while transformed fibroblasts (panel B) show rosettes (white arrow), filopodia (gray arrows) and lamellipodia/membrane ruffles (black arrow). 


\section{Src activation via $\mathrm{SH} 3$ engagement can result in cell transformation.}

Cell transformation in response to Src activation can be accomplished through the various mechanisms of Src activation. As mentioned above, dephosphorylation of the C-terminal tyrosine, either through mutation or activation of cellular phosphatases, results in transformation. Significantly, the activation of Src family kinases through SH3 engagement can also result in cell transformation. The HIV-1 protein Nef, which activates Hck through this mechanism, has been shown to transform fibroblasts upon co-expression with Hck (Briggs et al., 1997). Likewise, the Tip protein from Herpesvirus saimiri strain $484 \mathrm{C}$ has been shown to activate Lck in an SH3dependent manner (Lund et al., 1997; Lund et al., 1999). Additionally, Tip is required for the ability of this Herpesvirus to induce lymphomas in common marmosets (Duboise et al., 1998). As per definition, the transformation induced by each of these proteins is characterized by the reorganization of the actin cytoskeleton, signifying an increased potential for cell migration.

\section{cSrc is deregulated in many cancers, particularly metastatic tumors.}

Examination of the role cSrc plays in several tumor types supports the capability of $\mathrm{cSrc}$ to increase cell motility. Cell lines from metastatic colon carcinomas as well as biopsies from tumor metastases display increased cSrc kinase activity (Bolen et al., 1987; Cartwright et al., 1989; Cartwright et al., 1990; Rosen et al., 1986). Talamonti et al. demonstrated that the hyperactivation of cSrc in colon biopsies increased as disease state increased, with polyps of high malignant potential showing the smallest significant increase and distant metastases showing the highest (Talamonti et al., 1993). Similarly, cSrc is also activated in breast tumor cell lines (Jacobs and Rubsamen, 1983; Ottenhoff-Kalff et al., 1992; Rosen et al., 1986). cSrc is thought to promote breast cancer as it interacts with the Neu/HER2 receptor tyrosine kinase, the EGF receptor family member, which is a marker for poor prognosis (Maa et al., 1995). In 
support of this, cSrc potentiates EGF receptor signals and oncogenesis in cell lines. Mao et al. found that cSrc hyperactivity in metastases from colon carcinomas was directed by EGFR, Neu/HER2 and c-Met, the hepatocyte growth factor receptor (Mao et al., 1997). Although most studies have focused on cSrc in breast and colon cancers, cSrc has also been shown to be activated in other tumors including skin and some pediatric tumors (Barnekow et al., 1987; Rosen et al., 1986). Recent work has shown that adenovirus-mediated introduction of the negative cSrc regulator, CSK, into colon carcinoma cells inhibited cell migration and metastasis in nude mice, yet did not affect tumorigenicity (Nakagawa et al., 2000). As cSrc plays a crucial role in tumor metastasis, understanding the proteins and signals involved could provide insight valuable to the treatment of metastases.

\section{cSrc-mediated cytoskeletal rearrangements and subsequent promotion of tumor metastases involve PKC.}

One of the proteins which serves to direct cytoskeletal rearrangements and cell migration in response to cSrc activation is the Ca-dependent protein kinase, PKC. The PKC family is divided into 3 main groups, as determined by their requirements for efficient activation and phosphorylation of substrate (Toker, 1998). The classical PKC family is represented by PKC $\alpha$, $\beta$, and $\gamma$, while the novel PKC family consists of PKC $\delta, \varepsilon, \eta$ and $\theta$. Atypical PKCs are $\lambda / \imath$ and $\zeta$. Classical PKCs require calcium, diacyl-glycerol and phosphatidylserine for full activation, while novel PKCs are not responsive to calcium and require only diacyl-glycerol and phosphatidylserine. Atypical PKCs appear to require only phosphatidylserine. Many experiments studying PKC have relied on phorbol esters, which mimic diacyl-glycerol, to activate the classical and novel PKCs. cSrc activation has been reported to induce the activity of members of each of these PKC families (Qureshi et al., 1991; Zang et al., 1995). Upon PKC 
activation with phorbol esters, there is a depolymerization of actin filaments (Jaken et al., 1989), much like that seen upon cSrc activation. Furthermore, PKC has been shown to be involved in cell motility and other cytoskeleton-mediated functions. In a study involving $\mathrm{T}$ cells, it was shown that inhibition of PKC activity with staurosporine causes a decrease in $\mathrm{T}$ cell motility (Volkov et al., 1998). PKC activation with PMA was shown in another report to be essential for cell spreading and migration of a colon carcinoma cell line (Rigot et al., 1998). Similarly, inhibition of PKC was sufficient to impede the migration of cultured ovarian carcinoma cells (Szaniawska et al., 1998). In a study of nonmetastatic breast epithelial cells, Sun et. al demonstrated that expression of a PKC isoform, $\mathrm{PKC} \alpha$, decreased the cells' proliferation rate but increased the cells' motility and altered the cell phenotype to one consistent with motility (Sun and Rotenberg, 1999). These reports support the idea that PKC activity is essential for the phosphorylation-dependent cycling of cytoskeletal assembly/disassembly involved in cell motility. Further work with other proteins in the cSrc signaling cascade provides more evidence in support. The Ras oncogene is a downstream member of the cSrc signal cascade, and constitutive Ras activation results in a transformed cell morphology similar to that of cSrc. PKC has been shown to be activated in Ras-transformed cells (Morris et al., 1989). Furthermore, inhibition of PKC can block the transformed cell phenotype induced by Ras (Uberall et al., 1999). Several other studies have employed techniques to determine the role of specific PKC isoforms in Src transformation. A study of colonic adenocarcinoma cells showed that activation of either Ras or Src can upregulate and activate PKC $\alpha$, causing increased tumorigenicity in nude mice (Delage et al., 1993). cSrc has been implicated in the direct regulation of several PKC isoforms. Several PKC isoforms have been shown to be activated by tyrosine phosphorylation (Konishi et al., 1997) and at least one PKC isoform can associate directly with cSrc 
(Shanmugam et al., 1998). In fibroblasts, cSrc activation selectively activates PKC $\alpha$ and PKC delta (Zang et al., 1995). These data indicate that PKC isoforms are regulated in part by cSrc and may play a role in cSrc-mediated cytoskeletal rearrangements and tumor metastasis.

\section{AFAP-110 represents a potential effector protein for both cSrc and PKC-directed cytoskeletal rearrangements.}

Deletion experiments with cSrc, as described above, indicate one or more of its binding partners may be essential for the rearrangement of the cytoskeleton. PKC inhibition experiments have indicated it may play a role in cSrc-directed cytoskeletal effects through its kinase activity. The actin filament-associated protein, AFAP-110, is a Src binding partner and substrate with homology to PKC binding proteins, as described below. AFAP-110 fits several other criteria which indicate it has the potential to direct actin rearrangements in response to Src or PKC. The following dialogue is an in-depth description of prior research on AFAP-110, after which the hypothesis tested will be stated. The chapters which follow report the data gathered in testing the hypothesis and the conclusions drawn.

\section{Identification of AFAP-110 as a Src binding partner and substrate}

Upon its initial discovery, AFAP-110 was of significant interest because it formed a stable complex with activated forms of Src, such as vSrc or $\mathrm{Src}^{527 F}$, and could be detected as a co-immunoprecipitating protein with anti-phosphotyrosine antibodies via western blot analysis (Reynolds et al., 1989a). Early studies by Reynolds et al., demonstrated that the generation of 3 amino acid deletions within the $\mathrm{SH} 2$ or $\mathrm{SH} 3$ domains abrogated transformation potential of $\mathrm{Src}^{527 \mathrm{~F}}$ (Reynolds et al., 1989b). These same deletion constructs also failed to coimmunoprecipitate with AFAP-110 (Kanner et al., 1991). These data indicated that AFAP-110 was potentially an SH2 and SH3 binding partner for Src. Interestingly, SH3 deletion variants of 
$\operatorname{Src}^{527 \mathrm{~F}}\left(\mathrm{Src}^{\mathrm{d}-92 / 527 \mathrm{~F}}\right)$ also failed to phosphorylate AFAP-110, while SH2 deletion variants $\left(\mathrm{Src}^{\mathrm{dl}-}\right.$ 155/527F ) still retained an ability to phosphorylate AFAP-110 on tyrosine. These data indicated that perhaps SH3 interactions were important for presenting AFAP-110 for phosphorylation and that both $\mathrm{SH} 2$ and $\mathrm{SH} 3$ binding were important for forging stable complex formation with Src.

MAb 4C3 revealed that AFAP-110 was associated with stress filaments and the cell membrane in normal CEF cells (Reynolds et al., 1989a). In Src-transformed CEF cells, where the disruption of actin filament integrity is a hallmark for transformation (Felice et al., 1990; Reynolds et al., 1989a) and filaments are repositioned into "rosette-like" structures, AFAP-110 maintained a co-localization with these actin filament rosettes. These data indicated that perhaps AFAP-110 functioned by facilitating interactions between Src and actin filaments (Pawson, 1995). Previous data obtained by Hamaguchi and Hanafusa predicted that transformationcompetent forms of Src fractionated with the detergent-resistant cytoskeletal fraction, indicating that activated Src is closely associated with actin filaments (Hamaguchi and Hanafusa, 1987). Thus, AFAP-110 appeared positioned to facilitate this interaction, and Src/AFAP-110 interactions may have been important for affecting actin filament integrity in Src-transformed cells.

Parsons and colleagues first cloned the avian isoform of AFAP-110 and reported its sequence (Flynn et al., 1993)(GenBank accession \# L20303). Later, a neuronal-specific isoform of AFAP-110, called AFAP-120, was reported to contain an additional coding sequence near the carboxy terminus (Flynn et al., 1995)(GenBank accession \# L20302). More recently, the sequence of human AFAP-110 has been identified (Bai et al., submitted)(Human: GenBank accession \#AF188700). Other groups have also deposited partial sequences similar to AFAP110 (GenBank accession \#'s BB218408 and BB021778). Evidence supplied by Bai et al. as well 
as sequences deposited by the human genome project (http://www.ncbi.nlm.nih.gov) indicate that the gene for AFAP-110 is present on the distal part of the short arm of chromosome 4, at site 4p16.1 (also, see the human genome maps provided in Nature, volume 409, 2001, chromosome 4 map).

\section{AFAP-110 domain homology}

The predicted coding sequence of AFAP-110 indicates that it has a very high homology between species, e.g., 87\% identity between chicken and human (Bai et al., submitted). A functional domain structure can be predicted partly from the sequences and partly from experimental data. Here, AFAP-110 is predicted to contain an SH3 binding motif, two SH2 binding motifs, two pleckstrin homology domains, a leucine zipper motif and a strong target sequence for ser/thr phosphorylation (Figure 3). These regions contain several hypothetical binding sites that predict interactions with a variety of signaling proteins and/or lipids. The proteins of interest which will be detailed here are $\mathrm{cSrc}$ and PKC. These data indicate that AFAP-110 functions as an adaptor protein, which could link a variety of signaling proteins to actin filaments.

\section{A $\quad$ B C D D E}
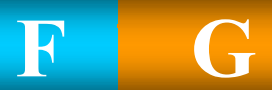

H

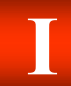

$\mathrm{J}$

Figure 3 Domain structure of AFAP-110. Schematic representation of AFAP-110 dividing the sequence into regions with homology to known structural/functional motifs. The amino-terminal end of the 729 residue sequence is depicted at the left. The domains shown are (residues): Aunique domain (1-68), B-SH3 binding motif (69-74), C-WW binding domain (75-84), D-SH2 binding motif (91-96), E-PH domain (153-248), F-multiple ser/thr kinase phosphorylation sites (250-346), G-PH domain (347-450), H-SH2 binding motif (451-455), I-leucine zipper/actin binding domain (511-637), J-carboxy-terminus (638-729). 


\section{SH3 interactions between AFAP-110 and Src family kinases}

Early studies by Ren et al. (Ren et al., 1993) and Yu et al. (Yu et al., 1992), demonstrated that an SH3 binding motif contained proline residues which formed the scaffolding structure of this motif, while surrounding hydrophobic amino acids were responsible for initiating specific contact with an SH3 domain (Weng et al., 1995). AFAP-110 contains two adjacent proline-rich motifs that resemble a consensus SH3-binding motif (Guappone and Flynn, 1997). Mutations that changed a single proline residue to alanine $\left(\mathrm{AFAP}^{71 \mathrm{~A}}\right)$ in the amino terminal proline-rich motif also abrogated the ability of the GST-SH3 ${ }^{\text {src }}$ fusion protein to affinity absorb AFAP ${ }^{71 \mathrm{~A}}$ from cell lysates, while mutating $\mathrm{Pro}^{77} \rightarrow \mathrm{Ala}^{77}$ in the second putative $\mathrm{SH} 3$ binding motif $\left(\mathrm{AFAP}^{77 \mathrm{~A}}\right.$ ) had no effect on affinity absorption (Guappone and Flynn, 1997). These data indicate that the amino-terminal, proline-rich SH3 binding motif was responsible for contacting the Src SH3 domain. This hypothesis is also supported by the observation that mutations in the Src SH3 domain prevent Src ${ }^{\mathrm{d} 192 / 527 \mathrm{~F}}$ from either phosphorylating or binding to AFAP-110 (Flynn et al., 1993; Kanner et al., 1991). Co-expression studies demonstrated that an AFAP ${ }^{71 \mathrm{~A}}$ mutant failed to form a stable complex with $\mathrm{Src}^{527 \mathrm{~F}}$, indicating a role for $\mathrm{SH} 3$ interactions in mediating stable complex formation. In addition, affinity absorption experiments using the Lyn or Fyn SH3 domains (GST-SH3 ${ }^{\text {lyn }}$ and GST-SH3 ${ }^{\text {fyn }}$ ) demonstrated that these fusion proteins could also affinity absorb AFAP-110; however, the cYes SH3 domain (GST-SH3 ${ }^{\text {yes }}$ ) failed to absorb cellular AFAP-110. These data indicate a mechanism for generating specificity in signaling among Src family kinases via SH3 interactions. Further experiments examined this hypothesis by predicting that activated cYes would also fail to interact with AFAP-110. Interestingly, it had been previously demonstrated that vYes failed to form a stable complex with AFAP-110 and was deficient in the phosphorylation of AFAP-110 (Kanner et al., 1990). Chimeric constructs of 
$\mathrm{Src}^{527 \mathrm{~F}} / \mathrm{cYes}$ in which the $\mathrm{Src}^{527 \mathrm{~F}}$ kinase domain was fused to the SH4-Unique-SH3-SH2 domains of cYes were also unable to form a stable complex with AFAP-110. Significantly, chimeric constructs in which only the SH3 domain of cYes was substituted into $\mathrm{Src}^{527 \mathrm{~F}}$ also failed to form a stable complex with AFAP-110 (Summy et al., 2000). These data indicate that SH3 interactions are required for stable complex formation with AFAP-110 and that specificity in signaling between Src family kinases might be achieved partly through differential SH3 binding partners.

\section{Src phosphorylates AFAP-110 to facilitate SH2 interactions}

AFAP-110 is a tyrosine-phosphorylated substrate for $\mathrm{Src}^{527 \mathrm{~F}}$ in vitro and in vivo (Flynn et al., 1992; Flynn et al., 1993). AFAP-110 contains 6 potential SH2 binding motifs which include a phosphotyrosine residue necessary for establishing an interaction with an $\mathrm{SH} 2$ domain (Guappone et al., 1998). Using site-directed mutagenesis, the individual tyrosine residues were mutated independently or in tandem. Affinity absorption studies and coexpression/immunoprecipitation studies with these mutants indicated AFAP-110 has more than one functional SH2 binding motif -- one amino terminal and one or two carboxy terminal motifs. Hypothetically, having more than one SH2 binding motif could facilitate the binding of two Src proteins to one AFAP-110 protein, resulting in the creation of larger signaling complexes. Alternatively, the different SH2 binding motifs may permit transit of a single $\mathrm{Src}^{527 \mathrm{~F}}$ protein upon AFAP-110, possibly assisting in transporting $\mathrm{Src}^{527 \mathrm{~F}}$ to a specific subcellular localization. A similar hypothesis has been presented by Fincham et al. in which phosphatidylinositol 3kinase (PI 3-kinase) can either facilitate, or is associated with, the transport of $\mathrm{Src}^{527 \mathrm{~F}}$ into focal adhesion structures (Fincham et al., 2000). Lastly, it is possible that the multiple sites of tyrosine 
phosphorylation may also assist in bringing activated Src in proximity to other SH2 domaincontaining proteins.

AFAP-110 hyperphosphorylation in Src transformed cells correlates with a change in AFAP-110 self-association, which may be independent of tyrosine phosphorylation

Sequence analysis of AFAP-110 indicated the presence of a leucine zipper motif, an alpha-helical structure of around 40 residues in length characterized by the presence of a leucine every seventh residue. These structural elements have been shown to facilitate protein homoand hetero-dimerization. To determine if AFAP-110 had the potential to dimerize via this motif, Qian et al. used a fusion protein construct containing the leucine zipper and surrounding region (GST-511-637) to affinity absorb AFAP-110 from cell lysate (Qian et al., 1998). This was shown to be the case, indicating AFAP-110 can self-associate. The absorption of AFAP-110 by this fusion protein is decreased upon co-expression of $\mathrm{Src}^{527 \mathrm{~F}}$ with AFAP-110, indicating a change in the self-association status of AFAP-110. The authors hypothesized that this change in self-association was likely due to a conformational change in AFAP-110, as a result of either phosphorylation or protein-protein interactions. This hypothesis was verified using gel filtration analysis, whereby cellular AFAP-110 fractionated in at least three separate peaks, representing multimers (such as tetramers and trimers), as well as a population of monomers. However, coexpression of $\mathrm{Src}^{527 \mathrm{~F}}$ caused AFAP-110 to fractionate in a single peak that also contained bound $\mathrm{Src}^{527 \mathrm{~F}}$ (Qian et al., 1998). Based on the molecular weight of the AFAP-110/Src ${ }^{527 \mathrm{~F}}$ complexes within this single peak, it was predicted that AFAP-110 would exist as either a dimer or a trimer, bound to two or one Src ${ }^{527 F}$ molecule, respectively. As AFAP-110 is hyperphosphorylated on tyrosine, serine and threonine residues in Src-transformed cells, the role of hyperphosphorylation in directing this change in conformation was investigated. Interestingly, it appears that the 
mechanism by which $\mathrm{Src}^{527 \mathrm{~F}}$ affects multimerization of AFAP-110 occurs independently of tyrosine phosphorylation, as was indicated by similar affinity absorptions with cSrc. GST-511637 fusion proteins were able to affinity absorb cellular AFAP-110 that had been co-expressed with cSrc (Qian et al., 1998). The cSrc kinase maintains a low, but significantly detectable level of tyrosine kinase activity (Hamaguchi and Hanafusa, 1987) and is able to phosphorylate AFAP110 in vivo (Reynolds et al., 1989a); however, cSrc is unable to activate cellular signals that initiate transformation or alter actin filament integrity (Reynolds et al., 1987). These data indicate that phosphorylation on tyrosine by cSrc did not alter the conformation of AFAP-110. However, it may have been possible that cSrc was unable to phosphorylate a specific and key tyrosine residue, one that may have only been phosphorylated in the presence of $\mathrm{Src}^{527 \mathrm{~F}}$. To test this, the $\mathrm{AFAP}^{5 \mathrm{Y}}$ mutant was co-expressed with $\mathrm{Src}^{527 \mathrm{~F}}$ and the ability of GST-511-637 to affinity absorb $\mathrm{AFAP}^{5 \mathrm{Y}}$ tested. Here, it was predicted that if tyrosine phosphorylation were responsible for altering the conformation of AFAP-110, then $\mathrm{AFAP}^{5 \mathrm{Y}}$ could be affinity absorbed by GST-511-637 in the presence of $\mathrm{Src}^{527 \mathrm{~F}}$ because it would not be phosphorylated on tyrosine. Conversely, if $\mathrm{AFAP}^{5 \mathrm{Y}}$ could not be absorbed, then perhaps the effect on AFAP-110 conformation was independent of tyrosine phosphorylation. The data demonstrated that GST511-637 was unable to affinity absorb $\mathrm{AFAP}^{5 \mathrm{Y}}$ in the presence of $\mathrm{Src}^{527 \mathrm{~F}}$, while in the absence of $\mathrm{Src}^{527 F}$ GST-511-637 could affinity absorb $\mathrm{AFAP}^{5 \mathrm{Y}}$. These data indicate that tyrosine phosphorylation is not responsible for affecting AFAP-110 conformation and that another cellular signal, directed by $\mathrm{Src}^{527 \mathrm{~F}}$, may be affecting the structure of AFAP-110 and preventing affinity absorption by GST-511-637. This signal may therefore involve ser/thr phosphorylation. These data indicate that conformational changes induced upon AFAP-110 by $\mathrm{Src}^{527 \mathrm{~F}}$ occur indirectly, possibly in response to ser/thr kinase activation downstream of $\mathrm{Src}^{527 \mathrm{~F}}$. 


\section{Deletion of the leucine zipper results in a change in self-association and reveals AFAP-110 has the intrinsic ability to direct cytoskeletal rearrangements}

To further examine the role of the leucine zipper in directing the self-association of AFAP-110, a mutant lacking the leucine zipper was constructed (AFAP-110 ${ }^{\Delta \text { zip }}$ ). Gel filtration analysis of this mutant revealed that it was found as a population of dimers, similar to that seen upon co-expression of wild-type AFAP-110 with $\operatorname{Src}^{527 \mathrm{~F}}$ (Qian et al., 1998). As this multimeric form of AFAP-110 had correlated with a transformed phenotype in previous experiments, this mutant was expressed to further evaluate this correlation. Expression of this mutant revealed that the cells containing the mutant displayed a phenotype which resembled Src transformation. These cells displayed a decrease in actin filaments and numerous rosettes, lamellipodia and membrane ruffles. Thus, AFAP-110 has the intrinsic potential to direct cytoskeletal rearrangements; however whether these effects are indirect via protein interactions or direct via interactions with actin remained untested.

\section{The amino-terminal PH domain of AFAP-110 may direct interactions with PKC}

The correlation of changes in both AFAP-110 self-association and serine/threonine phosphorylation of AFAP-110 with cytoskeletal rearrangements indicated AFAP-110 serine/threonine phosphorylation may play an important role in AFAP-110 function. Examination of AFAP-110 revealed two pleckstrin homology domains, structures which are known to direct interactions with serine/threonine kinases. Pleckstrin homology domains comprise a family of modular domains of around 100 residues found in a variety of proteins that have been shown to facilitate molecular interactions with either proteins or phospholipids (Shaw, 1996). PH domains, like most other modular binding domains, vary greatly from one protein to another, in respect to binding specificity and primary sequence. The tertiary structure of $\mathrm{PH}$ 
domains, however, is relatively well conserved, consisting of 7 beta strands followed by an alpha-helical region. The 7 beta strands form two beta sheets which meet at one long edge, resembling a book which is partially opened. The alpha helix is positioned in the opening between the two opposite ends of the sheets, effectively fitting in the "open book". Interactions, largely hydrophobic, between backbone and side-chain atoms stabilize the entire structure. Several PH domains contain an additional short alpha helix in one of the loops intervening between two beta strands. The diversity of primary sequence appears to lend to the ability to bind other molecules with variable affinities, when comparing different $\mathrm{PH}$ domains. For example, phospholipid binding affinities vary greatly between individual PH domains, with the PH domain from PLC- $\delta 1$ preferring Ins-(1,4,5)P3 and PtdIns(4,5)P2, while others like the PH domains from Btk and PKB prefer PtdIns(3,4,5)P3 (Gray et al., 1999; Kojima et al., 1997; Lemmon et al., 1995). Similar disparities for protein binding are seen among protein binding partners for PH domains (Rodriguez et al., 1999). Among the proteins which have been shown to bind $\mathrm{PH}$ domains are serine/threonine kinases $\mathrm{PKC}$, etc., WD40-repeat containing proteins RACK1, G $\beta \gamma$, others. The large, globular structure of the PH domain may permit the concomitant binding of several of these binding partners as well. This has been demonstrated for the WD40-repeat containing proteins (whose binding relies heavily on the alpha-helix of the $\mathrm{PH}$ domain) and $\mathrm{PKC}$, which binds to an opposite face of the PH domain involving the $2^{\text {nd }}-4^{\text {th }}$ beta strands. Thus the role of $\mathrm{PH}$ domains may be to approximate binding partners for the formation of signaling complexes.

AFAP-110 contains two potential PH domains which flank a stretch containing several putative sites for serine/threonine phosphorylation. Comparison of these PH domains' sequences shows that the amino-terminal PH domain shares highest homology with PH domains from $\beta$ - 
spectrin and Dynamin, which are also thought to direct interactions with PKCs, while the carboxy-terminal PH domain shares highest homology with the Btk PH domain. The aminoterminal $\mathrm{PH}$ domain also shares homology with the $\mathrm{PH}$ domain from Btk, which has been extensively investigated for its ability to interact with PKC. A modeling of the structure of this $\mathrm{PH}$ domain of AFAP-110 was constructed, based on the structure of the $\beta$-spectrin PH domain, as solved by NMR (Macias et al., 1994). This structure and the sequence homology it was based upon were used to determine the residues of the PH domain of AFAP-110 which likely correspond to the specific structural elements conserved in PH domains. Table 1 shows that the regions of homology between the amino-terminal PH domain of AFAP-110 and the PH domains of $\beta$-spectrin, Dynamin and Btk are highest for the first beta sheet, which includes strands 1-4. This sheet forms one side of the "book" structure and provides a continuous face which likely binds to PKC. The sequences of Spectrin, Dynamin, and Btk are shown in this table, as these domains have been well studied for their ability to bind both PKC and phosphoinositides. The consensus PKC binding sequence presented, however, also considers several other PH domains which also bind PKC. The amino-terminal PH domain of AFAP-110 fits this consensus well, with 7 out of 8 residues matching. If we include the buried hydrophobic residues indicated in this table with *, this number becomes 14 out of 15 . This table also considers the carboxyterminal PH domain of AFAP-110, which matches 4 out of 8 non-buried residues, and 10 out of 15 total consensus residues. A model of the amino-terminal PH domain of AFAP-110 and the potential binding sites for partners, including PKC, are shown in Figure 4. This figure includes the PH domain of Spectrin for comparison. Thus, it appears that one or both of the PH domains of AFAP-110 may direct interactions with PKC. 


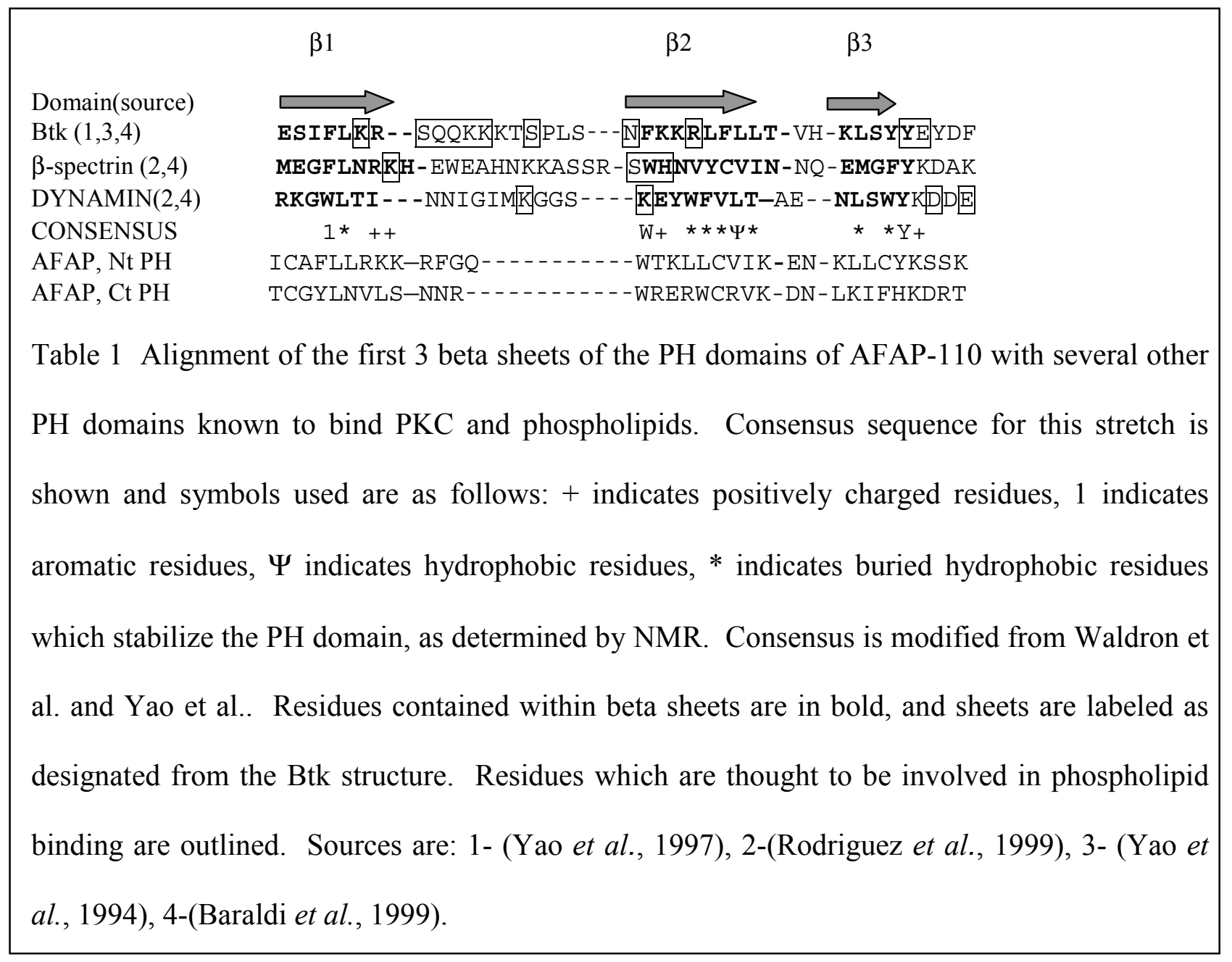




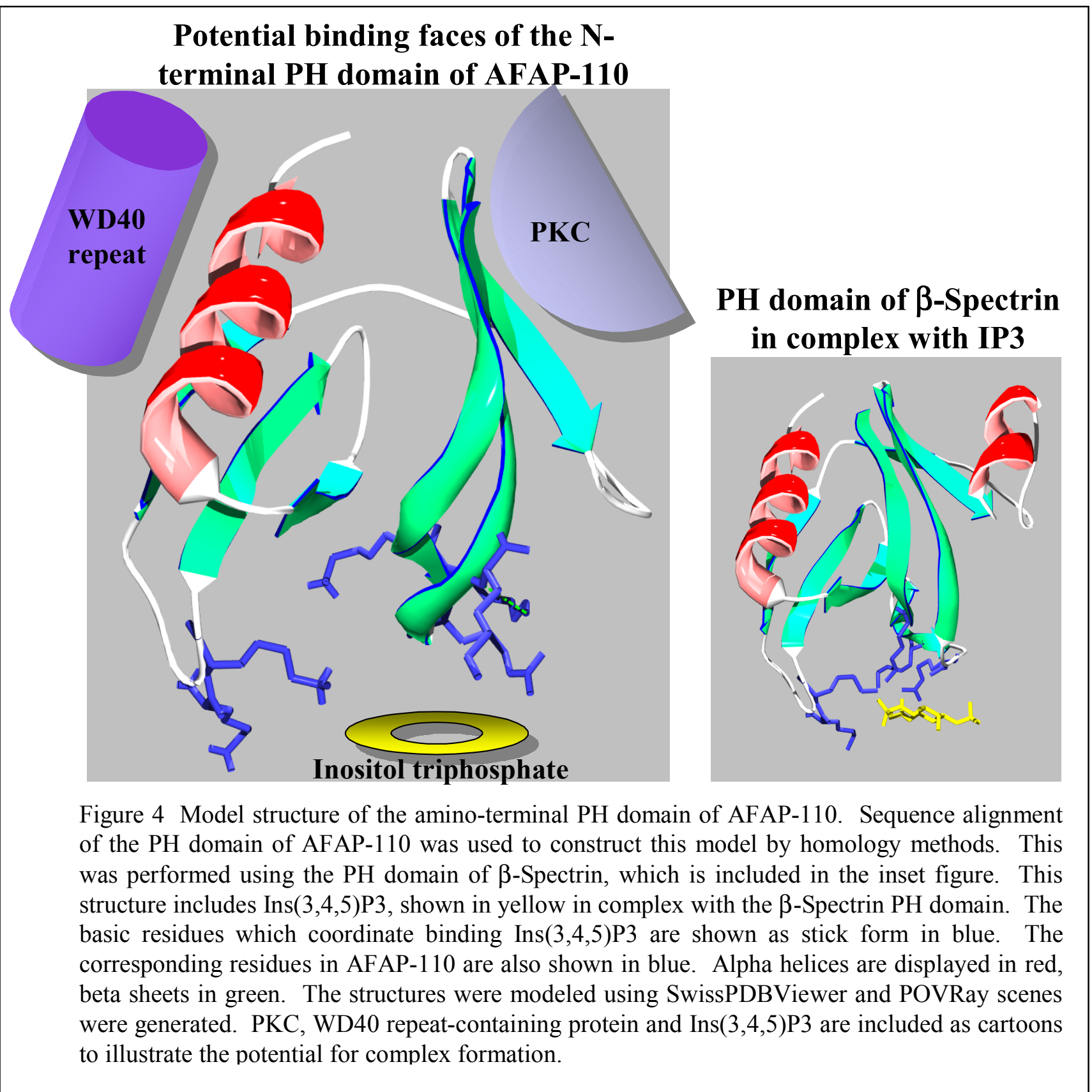




\section{Hypothesis}

Previous work on AFAP-110 has permitted the generation of a hypothesis that would implicate AFAP-110 in cytoskeletal rearrangements in response to cellular signals. This hypothesis predicts that AFAP-110 can direct cytoskeletal rearrangements by an indirect mechanism which is dependent upon the PH domain-directed phosphorylation of AFAP-110. More specifically, the phosphorylation of AFAP-110 induces a conformational change in AFAP110. This conformational change reveals an intrinsic ability for AFAP-110 to activate Src family kinases, thereby inducing cytoskeletal rearrangements in an indirect manner. This dissertation begins examining this hypothesis by investigating the intrinsic ability of AFAP-110 to induce alterations in actin filament structures as well as its ability to bind actin filaments directly in Chapter 1. The ability of AFAP-110 to direct cytoskeletal reorganization by an indirect mechanism involving the activation of Src is reported in Chapter 2. Chapter 3 details the mechanism which reveals the ability of AFAP-110 to activate Src in response to cellular signals. Overall conclusions and a model to accommodate these conclusions are presented in the discussion following these chapters. 


\section{References}

Baraldi,E, Carugo,KD, Hyvonen,M, Surdo,PL, Riley,AM, Potter,BV, O'Brien,R, Ladbury,JE, and Saraste,M. (1999). Structure. Fold. Des, 7, 449-460.

Barnekow,A, Paul,E, and Schartl,M. (1987). Cancer Res., 47, 235-240.

Barois,N, Forquet,F, and Davoust,J. (1998). J. Cell Sci., 111 ( Pt 13), 1791-1800.

Bell,L, Luthringer,DJ, Madri,JA, and Warren,SL. (1992). J. Clin. Invest, 89, 315-320.

Bolen,JB, Veillette,A, Schwartz,AM, DeSeau,V, and Rosen,N. (1987). Proc. Natl. Acad. Sci. U. S. $A, \mathbf{8 4}, 2251-2255$.

Boyce,BF, Yoneda,T, Lowe,C, Soriano,P, and Mundy,GR. (1992). J. Clin. Invest, 90, 16221627.

Briggs,SD, Sharkey,M, Stevenson,M, and Smithgall,TE. (1997). J. Biol. Chem., 272, 1789917902.

Brown,MT and Cooper,JA. (1996). Biochim. Biophys. Acta, 1287, 121-149.

Brugge,JS and Erikson,RL. (1977). Nature, 269, 346-348.

Cartwright,CA, Kamps,MP, Meisler,AI, Pipas,JM, and Eckhart,W. (1989). J. Clin. Invest, 83, 2025-2033.

Cartwright,CA, Meisler,AI, and Eckhart,W. (1990). Proc. Natl. Acad. Sci. U. S. A, 87, 558-562.

Collett,MS, Brugge,JS, and Erikson,RL. (1978). Cell, 15, 1363-1369.

Collette,Y, Arold,S, Picard,C, Janvier,K, Benichou,S, Benarous,R, Olive,D, and Dumas,C. (2000). J. Biol. Chem., 275, 4171-4176.

Delage,S, Chastre,E, Empereur,S, Wicek,D, Veissiere,D, Capeau,J, Gespach,C, and Cherqui,G. (1993). Cancer Res., 53, 2762-2770.

Duboise,SM, Lee,H, Guo,J, Choi,JK, Czajak,S, Simon,M, Desrosiers,RC, and Jung,JU. (1998). J. Virol., 72, 2607-2614.

Englund,PT. (1993). Annu. Rev. Biochem., 62, 121-138.

Felice,GR, Eason,P, Nermut,MV, and Kellie,S. (1990). Eur. J. Cell Biol., 52, 47-59.

Fincham,VJ, Brunton,VG, and Frame,MC. (2000). Mol. Cell Biol., 20, 6518-6536.

Flynn,DC, Koay,TC, Humphries,CG, and Guappone,AC. (1995). J. Biol. Chem., 270, 38943899. 
Flynn,DC, Leu,TH, Reynolds,AB, and Parsons,JT. (1993). Mol. Cell Biol., 13, 7892-7900.

Flynn,DC, Schaller,MD, and Parsons,JT. (1992). Oncogene, 7, 579-583.

Giancotti,FG and Ruoslahti,E. (1999). Science, 285, 1028-1032.

Gonfloni,S, Williams,JC, Hattula,K, Weijland,A, Wierenga,RK, and Superti-Furga,G. (1997). EMBO J., 16, 7261-7271.

Gray,A, Van Der,KJ, and Downes,CP. (1999). Biochem. J., 344 Pt 3, 929-936.

Guappone,AC and Flynn,DC. (1997). Mol. Cell Biochem., 175, 243-252.

Guappone,AC, Weimer,T, and Flynn,DC. (1998). Mol. Carcinog., 22, 110-119.

Gumbiner,BM. (1993). Neuron, 11, 551-564.

Hall,CL, Lange,LA, Prober,DA, Zhang,S, and Turley,EA. (1996). Oncogene, 13, 2213-2224.

Hamaguchi,M and Hanafusa,H. (1987). Proc. Natl. Acad. Sci. U. S. A, 84, 2312-2316.

Hartley,DA, Amdjadi,K, Hurley,TR, Lund,TC, Medveczky,PG, and Sefton,BM. (2000). Virology, 276, 339-348.

Hartley,DA, Hurley,TR, Hardwick,JS, Lund,TC, Medveczky,PG, and Sefton,BM. (1999). J. Biol. Chem., 274, 20056-20059.

Horne,WC, Neff,L, Chatterjee,D, Lomri,A, Levy,JB, and Baron,R. (1992). J. Cell Biol., 119, 1003-1013.

Hulett,MD and Hogarth,PM. (1994). Adv. Immunol., 57, 1-127.

Jacobs,C and Rubsamen,H. (1983). Cancer Res. , 43, 1696-1702.

Jaken,S, Leach,K, and Klauck,T. (1989). J. Cell Biol., 109, 697-704.

Kanner,SB, Reynolds,AB, Vines,RR, and Parsons,JT. (1990). Proc. Natl. Acad. Sci. U. S. A, 87, 3328-3332.

Kanner,SB, Reynolds,AB, Wang,HC, Vines,RR, and Parsons,JT. (1991). EMBO J., 10, 16891698.

Kaplan,KB, Swedlow,JR, Morgan,DO, and Varmus,HE. (1995). Genes Dev., 9, 1505-1517.

Kazlauskas,A. (1994). Curr. Opin. Genet. Dev., 4, 5-14.

Kojima,T, Fukuda,M, Watanabe,Y, Hamazato,F, and Mikoshiba,K. (1997). Biochem. Biophys. Res. Commun., 236, 333-339. 
Konishi,H, Tanaka,M, Takemura,Y, Matsuzaki,H, Ono,Y, Kikkawa,U, and Nishizuka,Y. (1997). Proc. Natl. Acad. Sci. U. S. A, 94, 11233-11237.

Lemmon,MA, Ferguson,KM, O'Brien,R, Sigler,PB, and Schlessinger,J. (1995). Proc. Natl. Acad. Sci. U. S. A, 92, 10472-10476.

Levinson,AD, Oppermann,H, Levintow,L, Varmus,HE, and Bishop,JM. (1978). Cell, 15, 561572.

Lund,T, Medveczky,MM, and Medveczky,PG. (1997). J. Virol., 71, 378-382.

Lund,TC, Prator,PC, Medveczky,MM, and Medveczky,PG. (1999). J. Virol., 73, 1689-1694.

Maa,MC, Leu,TH, McCarley,DJ, Schatzman,RC, and Parsons,SJ. (1995). Proc. Natl. Acad. Sci. U. S. $A, 92,6981-6985$.

Macias,MJ, Musacchio,A, Ponstingl,H, Nilges,M, Saraste,M, and Oschkinat,H. (1994). Nature, 369, 675-677.

Maidment,SL. (1997). Anticancer Res., 17, 4145-4149.

Mao,W, Irby,R, Coppola,D, Fu,L, Wloch,M, Turner,J, Yu,H, Garcia,R, Jove,R, and Yeatman,TJ. (1997). Oncogene, 15, 3083-3090.

Moarefi,I, LaFevre-Bernt,M, Sicheri,F, Huse,M, Lee,CH, Kuriyan,J, and Miller,WT. (1997). Nature, 385, 650-653.

Morris,JD, Price,B, Lloyd,AC, Self,AJ, Marshall,CJ, and Hall,A. (1989). Oncogene, 4, 27-31.

Nakagawa,T, Tanaka,S, Suzuki,H, Takayanagi,H, Miyazaki,T, Nakamura,K, and Tsuruo,T. (2000). Int. J. Cancer, 88, 384-391.

Ottenhoff-Kalff,AE, Rijksen,G, van Beurden,EA, Hennipman,A, Michels,AA, and Staal,GE. (1992). Cancer Res., 52, 4773-4778.

Pawson,T. (1995). Nature, 373, 573-580.

Petty,HR, Kindzelskii,AL, Adachi,Y, and Todd,RF, III. (1997). J. Pharm. Biomed. Anal., 15, 1405-1416.

Purchio,AF, Erikson,E, Brugge,JS, and Erikson,RL. (1978). Proc. Natl. Acad. Sci. U. S. A, 75, 1567-1571.

Qian,Y, Baisden,JM, Westin,EH, Guappone,AC, Koay,TC, and Flynn,DC. (1998). Oncogene, 16, 2185-2195.

Qureshi,SA, Joseph,CK, Rim,M, Maroney,A, and Foster,DA. (1991). Oncogene, 6, 995-999.

Ren,R, Mayer,BJ, Cicchetti,P, and Baltimore,D. (1993). Science, 259, 1157-1161. 
Reynolds,AB, Kanner,SB, Wang,HC, and Parsons,JT. (1989a). Mol. Cell Biol., 9, 3951-3958.

Reynolds,AB, Roesel,DJ, Kanner,SB, and Parsons,JT. (1989b). Mol. Cell Biol., 9, 629-638.

Reynolds,AB, Vila,J, Lansing,TJ, Potts,WM, Weber,MJ, and Parsons,JT. (1987). EMBO J., 6, 2359-2364.

Reynolds,PJ, Hurley,TR, and Sefton,BM. (1992). Oncogene, 7, 1949-1955.

Rigot,V, Lehmann,M, Andre,F, Daemi,N, Marvaldi,J, and Luis,J. (1998). J. Cell Sci., 111 ( Pt 20), 3119-3127.

Rodier,JM, Valles,AM, Denoyelle,M, Thiery,JP, and Boyer,B. (1995). J. Cell Biol., 131, 761773.

Rodriguez,MM, Ron,D, Touhara,K, Chen,CH, and Mochly-Rosen,D. (1999). Biochemistry, 38, 13787-13794.

Rosen,N, Bolen,JB, Schwartz,AM, Cohen,P, DeSeau,V, and Israel,MA. (1986). J. Biol. Chem., 261, 13754-13759.

Schwartzberg,PL, Xing,L, Hoffmann,O, Lowell,CA, Garrett,L, Boyce,BF, and Varmus,HE. (1997). Genes Dev., 11, 2835-2844.

Shanmugam,M, Krett,NL, Peters,CA, Maizels,ET, Murad,FM, Kawakatsu,H, Rosen,ST, and Hunzicker-Dunn,M. (1998). Oncogene, 16, 1649-1654.

Shaw,G. (1996). Bioessays, 18, 35-46.

Soriano,P, Montgomery,C, Geske,R, and Bradley,A. (1991). Cell, 64, 693-702.

Summy,JM, Guappone,AC, Sudol,M, and Flynn,DC. (2000). Oncogene, 19, 155-160.

Sun,XG and Rotenberg,SA. (1999). Cell Growth Differ., 10, 343-352.

Szaniawska,B, Gawrychowski,K, and Janik,P. (1998). Neoplasma, 45, 7-11.

Talamonti,MS, Roh,MS, Curley,SA, and Gallick,GE. (1993). J. Clin. Invest, 91, 53-60.

Tang,DG and Honn,KV. (1994). Invasion Metastasis, 14, 109-122.

Thomas,SM and Brugge,JS. (1997). Annu. Rev. Cell Dev. Biol., 13, 513-609.

Toker,A. (1998). Front Biosci., 3, D1134-D1147.

Uberall,F, Hellbert,K, Kampfer,S, Maly,K, Villunger,A, Spitaler,M, Mwanjewe,J, BaierBitterlich,G, Baier,G, and Grunicke,HH. (1999). J. Cell Biol., 144, 413-425. 
Valitutti,S, Dessing,M, Aktories,K, Gallati,H, and Lanzavecchia,A. (1995). J. Exp. Med., 181, $577-584$.

van Biesen,T, Luttrell,LM, Hawes,BE, and Lefkowitz,RJ. (1996). Endocr. Rev., 17, 698-714.

Volkov, Y, Long,A, and Kelleher,D. (1998). J. Immunol., 161, 6487-6495.

Weil,R and Veillette,A. (1994). J. Biol. Chem., 269, 22830-22838.

Weiss,A and Littman,DR. (1994). Cell, 76, 263-274.

Weng,Z, Rickles,RJ, Feng,S, Richard,S, Shaw,AS, Schreiber,SL, and Brugge,JS. (1995). Mol. Cell Biol., 15, 5627-5634.

Wright,DD, Sefton,BM, and Kamps,MP. (1994). Mol. Cell Biol., 14, 2429-2437.

Yao,L, Kawakami,Y, and Kawakami,T. (1994). Proc. Natl. Acad. Sci. U. S. A, 91, 9175-9179.

Yao,L, Suzuki,H, Ozawa,K, Deng,J, Lehel,C, Fukamachi,H, Anderson,WB, Kawakami,Y, and Kawakami,T. (1997). J. Biol. Chem., 272, 13033-13039.

Young,MA, Gonfloni,S, Superti-Furga,G, Roux,B, and Kuriyan,J. (2001). Cell, 105, 115-126.

Yu,H, Rosen,MK, Shin,TB, Seidel-Dugan,C, Brugge,JS, and Schreiber,SL. (1992). Science, 258, $1665-1668$.

Zang,Q, Frankel,P, and Foster,DA. (1995). Cell Growth Differ., 6, 1367-1373. 


\section{Chapter 1}

AFAP-110 binds actin directly and has the intrinsic ability to induce cytoskeletal rearrangements. 
AFAP-110 is a binding partner for Src which is found on the actin cytoskeleton in both non-transformed and transformed cells. SH2/SH3 deletion mutants of Sre fail to transform cells and fail to form a stable complex with AFAP-110, as well. To examine the potential involvement of AFAP-110 in the cytoskeletal rearrangements that are characteristic of Src transformation, AFAP-110 was studied for its potential to bind actin directly. Data presented here indicate that AFAP-110 binds actin filaments directly through a carboxyterminal region with homology to known actin-binding domains. Additionally, deletion of the leucine zipper motif of AFAP-110 results in a phenotype which resembles Src transformation. We conclude that AFAP-110 may play a role in cytoskeletal reorganizations through its dual potentials to both bind actin filaments directly and induce cytoskeletal reorganizations. 


\section{Introduction}

As AFAP-110 was originally defined by its association with actin, early work on AFAP110 was aimed toward implicating it in the reorganization of the actin cytoskeleton. Experiments toward that end were first reported in a manuscript published in 1998 by Qian et al. This work included a figure of immunfluorescence data (contributed by myself) which indicated that the expression of a specific mutant of AFAP-110 resulted in the reorganization of the actin cytoskeleton of Cos-1 cells similar to that seen upon expression of $\operatorname{Src}^{527 F}$ (Qian et al., 1998). The mutant used consisted of a deletion variant of AFAP-110 lacking the leucine zipper motif (residues 553-593), AFAP-110 ${ }^{\Delta \text { lzip }}$. This mutant was used in this manuscript to investigate the potential for AFAP-110 to dimerize via this motif. The experiments reported by Qian et al. indicated that AFAP-110 does indeed self-associate in part by this mechanism and that this selfassociation was affected by Src activation. FPLC analysis indicated that AFAP-110 shifted from a population of several multimeric forms to a single population upon co-expression of $\mathrm{Src}^{527 \mathrm{~F}}$. This shift to a single, dimeric population was similarly noted upon expression of AFAP-110 ${ }^{\Delta \text { lzip }}$ (Flynn, unpublished data). Thus, we asked if this change in self-association also correlated with the cytoskeletal rearrangements seen upon expression of $\mathrm{Src}^{527 \mathrm{~F}}$. AFAP-110 ${ }^{\Delta \text { lzip }}$ expressors indeed displayed cytoskeletal rearrangements which mimicked Src transformation. Thus AFAP110 displayed the potential to direct cytoskeletal rearrangements.

The co-localization of AFAP-110 to actin structures permitted the generation of the hypothesis that AFAP-110 may interact directly with actin and induce these cytoskeletal rearrangements directly. Experiments designed to test this hypothesis were reported by Qian et al. in a manuscript published in 2000. This manuscript described AFAP-110 as an actin-binding protein with the capability to induce cytoskeletal rearrangements in several cell types. The following is a discussion of my contributions to this manuscript. 


\section{Results}

Sequence analysis of the carboxy-terminal region of AFAP-110 revealed homology to known actin-binding motifs, as shown in Table 1. AFAP-110 contains homology to two distinct types of actin-binding domains in this region which were termed ABD1 and ABD3 by Taylor et al. (Taylor et al., 1998). The first of these

\section{TABLE 1}

Similarity among Actin Filament-binding Domains

\begin{tabular}{|c|c|c|c|c|c|c|c|c|c|c|c|c|c|c|c|}
\hline Similarity to ABD1 & & & & & & & & & & & & & & & \\
\hline AFAP-110 (avian, 601-615) & V & $\mathrm{N}$ & $\mathrm{A}$ & $\mathrm{G}$ & $\mathrm{R}$ & $\mathrm{K}$ & $\mathrm{T}$ & Q & $\mathrm{V}$ & I & $\mathrm{L}$ & $\mathrm{E}$ & $\mathrm{D}$ & $\mathrm{K}$ & $\mathrm{L}$ \\
\hline Dystrophin (human, 92-106) & V & $\mathrm{N}$ & I & G & $\mathrm{S}$ & $\mathrm{T}$ & D & I & V & D & G & $\mathrm{N}$ & $\mathrm{H}$ & $\mathrm{K}$ & $\mathrm{L}$ \\
\hline Dystrophin (avian, 96-110) & V & $\mathrm{N}$ & I & G & $\mathrm{S}$ & $\mathrm{S}$ & $\mathrm{D}$ & I & V & $\mathrm{D}$ & $\mathrm{G}$ & $\mathrm{N}$ & $\mathrm{H}$ & $\mathrm{K}$ & $\mathrm{L}$ \\
\hline$\alpha$-Actinin (dicty., 101-115) & V & $\mathrm{G}$ & I & $\mathrm{G}$ & A & $\mathrm{E}$ & $\mathrm{E}$ & $\mathrm{L}$ & V & $\mathrm{D}$ & $\mathrm{K}$ & $\mathrm{N}$ & $\mathrm{L}$ & $\mathrm{K}$ & $\mathrm{M}$ \\
\hline$\alpha$-actinin (avian, $120-134$ ) & V & $\mathrm{S}$ & I & $\mathrm{G}$ & $\mathrm{A}$ & $\mathrm{E}$ & $\mathrm{E}$ & $\mathrm{L}$ & V & $\mathrm{D}$ & $\mathrm{G}$ & $\mathrm{N}$ & $\mathrm{A}$ & $\mathrm{K}$ & M \\
\hline Spectrin (drosophila, 127-141) & $\mathrm{E}$ & $\mathrm{N}$ & I & G & $\mathrm{S}$ & $\overline{\mathrm{H}}$ & $\mathrm{K}$ & I & V & $\mathrm{D}$ & G & $\mathrm{N}$ & $\mathrm{A}$ & $\mathrm{S}$ & $\mathrm{L}$ \\
\hline Spectrin (human, 131-145) & $\mathrm{E}$ & $\mathrm{N}$ & $\mathrm{M}$ & G & $\mathrm{S}$ & $\mathrm{H}$ & $\mathrm{D}$ & I & V & $\mathrm{D}$ & $\mathrm{G}$ & $\mathrm{N}$ & $\mathrm{H}$ & $\mathrm{R}$ & $\mathrm{L}$ \\
\hline ABP-120 (dicty., 90-104) & $\mathrm{V}$ & $\mathrm{G}$ & I & $\mathrm{G}$ & A & $\mathrm{E}$ & $\mathrm{D}$ & I & V & D & $\mathrm{S}$ & Q & $\mathrm{L}$ & $\mathrm{K}$ & $\mathrm{L}$ \\
\hline $\mathrm{ABP}$ & V & $\mathrm{S}$ & I & $\mathrm{D}$ & $\mathrm{S}$ & $\mathrm{D}$ & $\mathrm{A}$ & I & V & $\mathrm{D}$ & $\mathrm{G}$ & $\mathrm{N}$ & $\mathrm{L}$ & $\mathrm{K}$ & $\mathrm{L}$ \\
\hline Filamin (122-136) & V & $\mathrm{S}$ & I & $\mathrm{D}$ & $\mathrm{S}$ & $\mathrm{K}$ & $\mathrm{A}$ & I & V & $\mathrm{D}$ & $\mathrm{G}$ & $\mathrm{N}$ & $\mathrm{L}$ & $\mathrm{K}$ & $\mathrm{L}$ \\
\hline Tensin (avian, 372-385) & $\mathrm{E}$ & $\mathrm{N}$ & $\mathrm{G}$ & $\mathrm{P}$ & S & $\mathrm{V}$ & S & V & - & $\mathrm{D}$ & $\mathrm{Y}$ & $\mathrm{N}$ & $\mathrm{T}$ & $\mathrm{S}$ & $\mathrm{D}$ \\
\hline Fimbrin (avian, 211-225) & $\mathrm{V}$ & $\mathrm{N}$ & I & G & $\tilde{S}$ & Q & $\mathrm{D}$ & $\mathrm{L}$ & Q & $\mathrm{E}$ & $\mathrm{G}$ & $\mathrm{K}$ & $\mathrm{P}$ & $\mathrm{H}$ & $\mathrm{L}$ \\
\hline T-plastin (human, 209-223) & V & $\mathrm{N}$ & I & G & A & $\mathrm{E}$ & $\mathrm{D}$ & $\mathrm{L}$ & $\mathrm{R}$ & $\vec{A}$ & $\mathrm{G}$ & $\mathrm{K}$ & $\mathrm{P}$ & $\mathrm{H}$ & $\mathrm{L}$ \\
\hline \multicolumn{16}{|l|}{ Similarity to ABD3 } \\
\hline AFAP-110 (614-617) & $\mathrm{K}$ & $\mathrm{L}$ & $\mathrm{K}$ & $\mathrm{K}$ & & & & & & & & & & & \\
\hline Thymosin $\beta 4(17-20)$ & $\mathrm{K}$ & $\mathrm{L}$ & $\mathrm{K}$ & $\mathrm{K}$ & & & & & & & & & & & \\
\hline Villin (818-821) & $\mathrm{N}$ & $\mathrm{L}$ & $\mathrm{K}$ & $\mathrm{K}$ & & & & & & & & & & & \\
\hline Vasp (234-237) & $\mathrm{K}$ & $\mathrm{L}$ & $\mathrm{R}$ & $\mathrm{K}$ & & & & & & & & & & & \\
\hline
\end{tabular}


domains, the ABD1, covers 15 amino acids (601-615), as shown in Table 1. Domains of this type from 12 other proteins are included for comparison. Overlapping this ABD1 region in AFAP-110 is the putative ABD3. This domain consists of 4 residues which have been shown to be responsible for actin-binding in several proteins. The $\mathrm{ABD} 3$ regions from these proteins are included in Table 1 for comparison to the potential ABD3 from AFAP-110. This motif consists of three basic residues and a hydrophobic leucine, for all these regions except for the ABD3 of Villin, which contains an asparagine in place of lysine seen in the other domains. The putative ABD3 from AFAP-110 fits this domain consensus exactly, as it is comprised of KLKK. It is interesting to note that this $\mathrm{ABD} 3$ overlaps the $\mathrm{ABD} 1$ in AFAP-110, with the KL residues that end the ABD1 providing the first half of the ABD3.

To address the potential for AFAP-110 to bind actin structures directly, several mutants were created which lacked portions of the carboxy-terminal region of AFAP-110. These mutants were used to test the idea that these putative actin-binding domains were involved in linking AFAP-110 to actin. Site-directed mutagenesis was employed to introduce stop codons into the coding sequence of AFAP-110 to create mutants which lacked the carboxy-terminal 176 amino acids (AFAP-110 $0^{\Delta 176}$ ), the carboxy-terminal 136 amino acids (AFAP-110 ${ }^{\Delta 136}$ ) or the carboxyterminal 114 amino acids (AFAP-110 ${ }^{\Delta 114}$ ). The names used here have been changed from the published manuscript as to more accurately reflect the nature of each mutant. Figure 1 includes a schematic representation of these mutants and divides the carboxy-terminal region of AFAP-110 into three sections: the coil/turn region, the leucine zipper motif and the alpha-helical region. As depicted, the mutants created covered these three regions, particularly the alpha-helical region. The expression and size of these mutants was verified by SDS-PAGE followed by western blot analysis. 


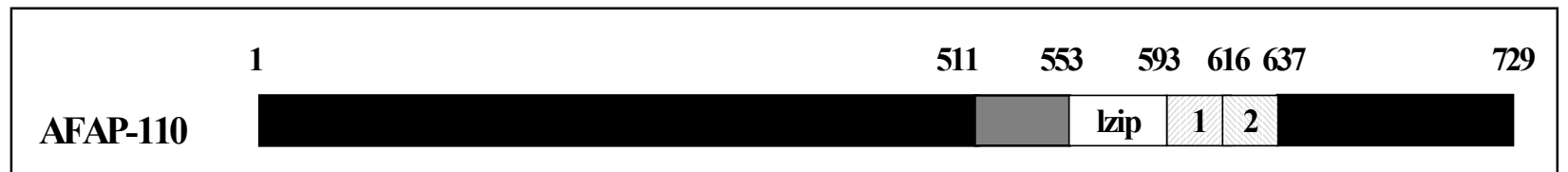

AFAP-110 1176
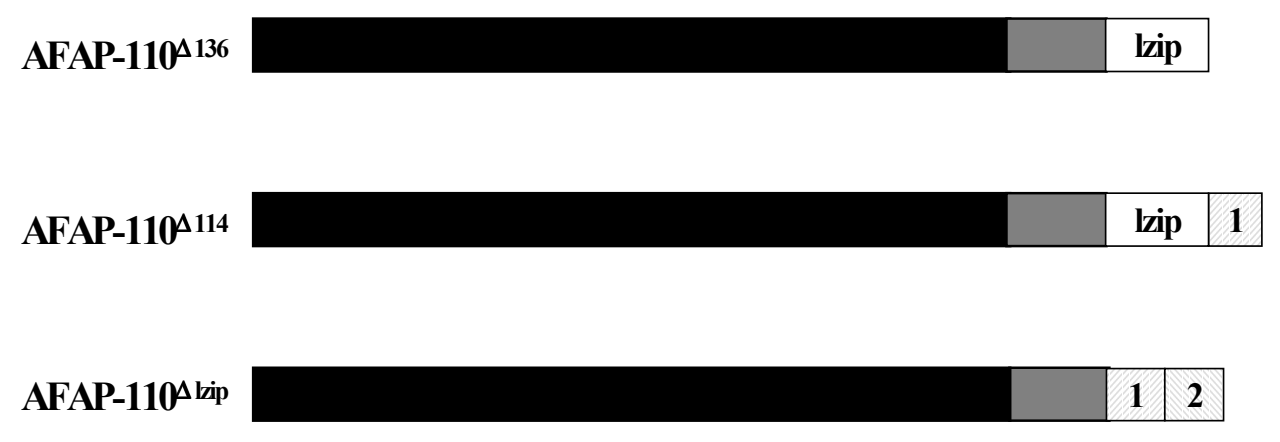

Figure 1 Deletion mutants of AFAP-110. The creation of these mutants is described in Materials and Methods. Numbers at the top designate the residues in AFAP-110 which are used here in reference to deletions. The leucine zipper motif of AFAP-110 (residues 553-593) is labeled lzip. The alpha helical region adjacent to the leucine zipper, here illustrated by the diagonal lines, is divided into two portions, labeled 1 (residues 593-615) and 2 (residues 616-637). Both portions of this region were deleted in AFAP- $110^{4176}$ and AFAP-110 136 . AFAP- $110^{\Delta 114}$, however, maintained the first half of this region as the protein was truncated at residue 615. This alpha helical region contains both putative actin binding domains of AFAP-110 and therefore these regions were deleted in these mutants. AFAP- $110^{\Delta 114}$ resulted in the deletion of only the second half of the second of these actin binding domains, which is found in residues 614-617.

The mutants created were expressed in C3H10T1/2 mouse fibroblasts to determine their ability to colocalize with actin structures. Transfection and microscopic analysis were carried out as described in Material and Methods. The first of the mutants shown, AFAP- $110^{\Delta 176}$ lacked the leucine zipper, the alpha-helical region and the rest of the carboxy-terminus. This mutant was shown to be deficient for binding actin, as seen in Figure 2, panels D-F. This was in contrast to wild-type AFAP-110, which colocalized with actin structures, as seen in Figure 2, panels A-C. As deletion of the leucine zipper did not appear to affect colocalization with actin structures (Qian et al., 1998), and the putative actin-binding domains were contained in the alpha-helical region, further deletions targeted this region. Deletion of this entire region (AFAP-110 ${ }^{\Delta 136}$ ) again resulted in abrogation of colocalization with actin structures, as seen in Figure 2, panels G- 
I. Another deletion, encompassing the carboxy-terminal 114 residues of AFAP-110 partially abrogated interactions with actin structures. This is seen in Figure 2, panels J-L, which indicates partial colocalization with actin filaments. Thus, the carboxy-terminal 136 residues of AFAP110 appear to be involved in the interaction between AFAP-110 and actin structures.

Figure 2 also reveals that residues 593-616 are involved in the membrane localization of AFAP-110. Wild-type AFAP-110 and AFAP- $110^{\Delta 114}$ colocalized with the cell membrane. AFAP- $110^{\Delta 176}$ and AFAP- $110^{\Delta 136}$ were both deficient for membrane localization, as highlighted with gray arrows in Figure 2. Thus, the common region deleted from these two mutants, residues 593-616, are likely involved in this property of AFAP-110.

To confirm that the carboxy-terminal region was responsible for the colocalization with actin structures, GFP-tagged forms of AFAP-110 were created and expressed in C3H10T1/2 fibroblasts. Cloning performed by Dr. Qian resulted in two forms of GFP-tagged AFAP-110 which placed the AFAP-110 coding sequence carboxy-terminal to and in frame with the GFP coding sequence, resulting in the expression of fusion proteins. These mutants were expressed and examined for colocalization with actin structures. Wild-type AFAP-110 fused to GFP was seen to colocalize with actin (Figure 3, panels A-C), while GFP alone failed to colocalize with actin (Figure 3, panels D-F). Furthermore, expression of the carboxy-terminal 219 amino-acids fused to GFP (GFP-Cterm) revealed colocalization with actin structures (Figure 3, panels G-I). This confirmed that the carboxy-terminal region is involved in the colocalization of AFAP-110 to actin structures. 

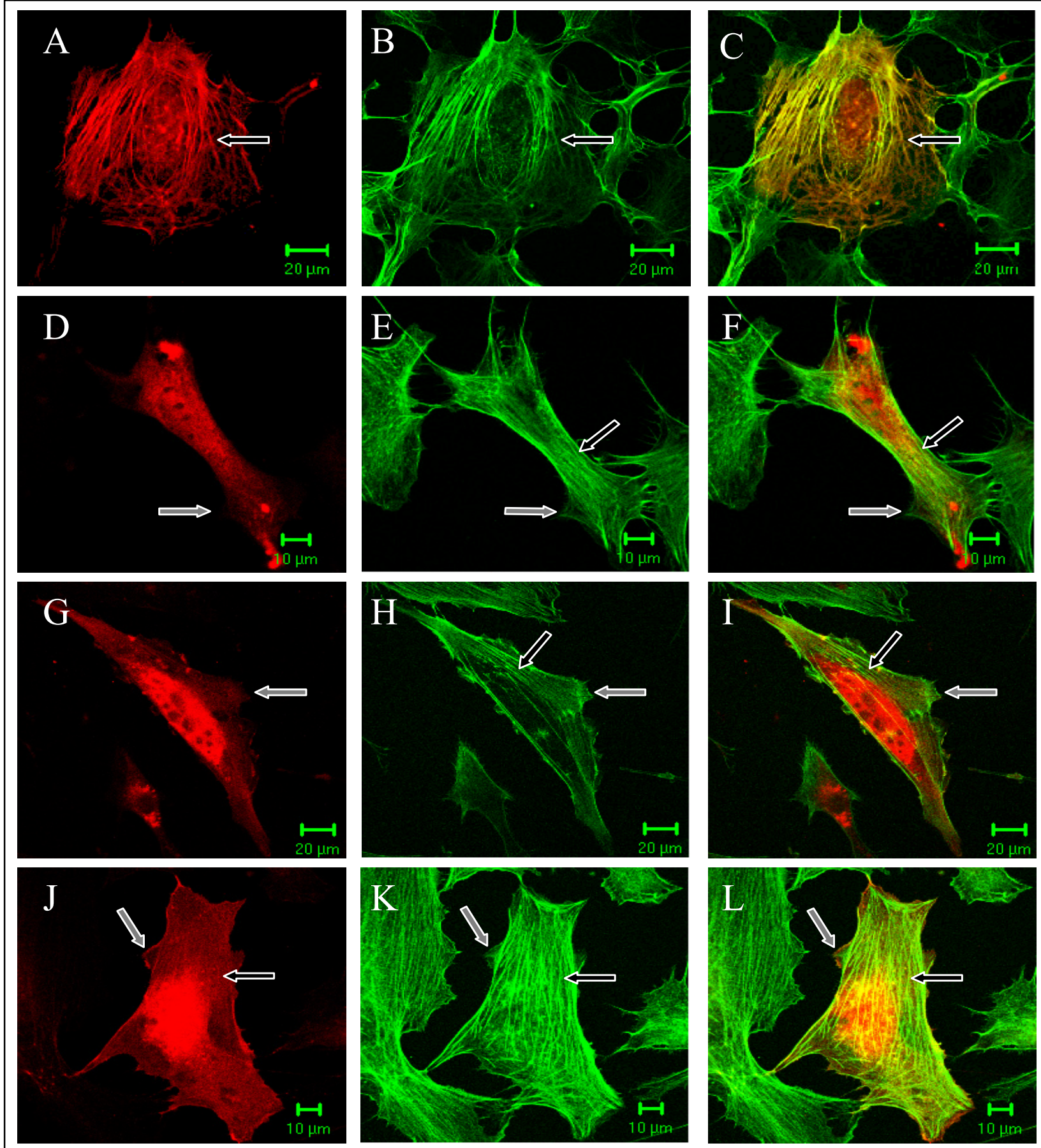

Figure 2 Deletions in the putative actin-binding region of AFAP-110 abrogate colocalization with actin filaments. AFAP-110 and mutants were expressed in C3H10T1/2 fibroblasts, as described in Materials and Methods. A, D, G and J demonstrate AFAP-110 labeling with mAb 4C3, while $\mathrm{B}, \mathrm{E}, \mathrm{H}$ and $\mathrm{K}$ convey actin filaments labeled with FITC phalloidin. The corresponding converged images of these panels are shown in C, F, I and L to demonstrate colocalization between AFAP-110 and actin, which appears yellow. Black arrows indicate actin filaments and colocalization of AFAP-110 with actin filaments. Gray arrows indicate the cell membrane and highlight the colocalization (or absence thereof) of AFAP-110 with the cell membrane. Cells were transfected with AFAP-110 or mutants as follows: A-C, Wild-type AFAP-110; D-F, AFAP-110 ${ }^{\Delta 176}$; G-I, AFAP-110 $0^{\Delta 136}$; J-L, AFAP-110 $0^{\Delta 114}$. 

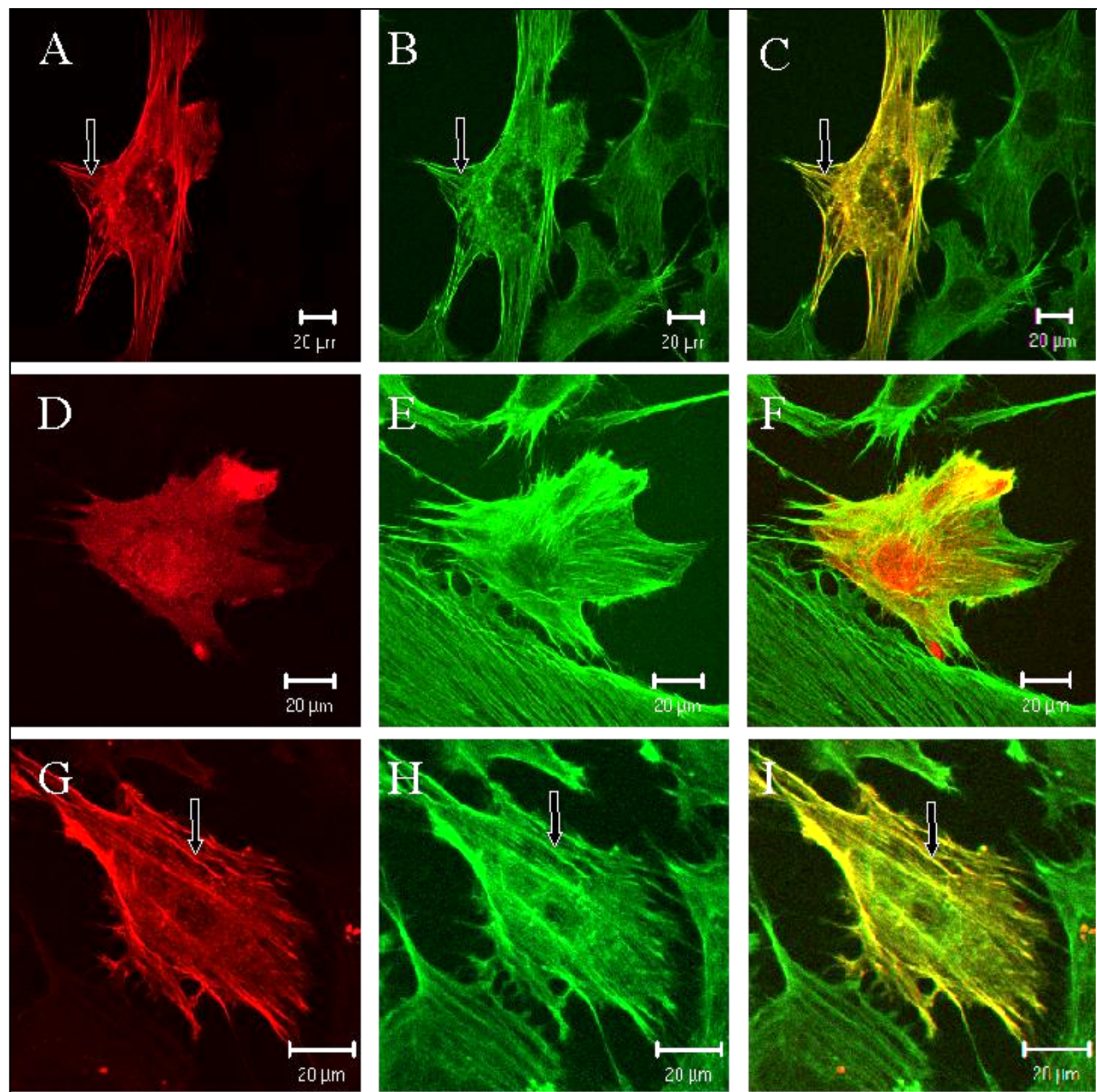

Figure 3 The carboxy-terminal 219 amino acids are sufficient for colocalization with actin filaments in vivo. GFP-tagged forms of AFAP-110 (panels A and G) as well as GFP alone (panel D) were expressed in C3H10T1/2 fibroblasts, as described in Materials and Methods. Cells were labeled to visualize actin filaments (panels B, E and $\mathrm{H}$ ). Converged images are shown in panels $\mathrm{C}, \mathrm{F}$ and I to illustrate colocalization, which appears yellow. Black arrows highlight this colocalization. Cells were transfected as follows: A-C, GFP-AFAP-110; D-F, GFP; G-I, GFP-Cterm. 
Immediately amino-terminal to the actin-binding domains of AFAP-110 is the leucine zipper motif. Both of these regions are predicted to exist as part of a long alpha-helix. It had been previously noted that expression of AFAP- $110^{\text {Slzip }}$ resulted in a phenotype which resembled Src-transformed cells (Qian et al., 1998). Cos-1 cells expressing this mutant were reported to have decreased actin filaments and an increase in actin rich rosettes with which AFAP-110 $0^{\Delta l z i p}$ colocalized. These results were recapitulated here, and the expression of this mutant in other cells was more closely examined. Figure 4 shows the effects of expression of AFAP- $110^{\Delta \text { Lzip }}$ on Cos-1, C3H10T1/2 and NIH3T3 fibroblasts. Panels A-C show that in Cos-1 cells, expression of AFAP- $110^{\Delta l z i p}$ resulted in the repositioning of actin filaments into rosettes and AFAP-110 1 szip colocalized with these structures (black arrows). Additionally, AFAP-110 $0^{\Delta \text { lzip }}$ colocalized with actin-rich structures at the cell membrane, such as filopodia, membrane ruffles and lamellipodia (white arrows). Panels D-F show that similar results were seen in C3H10T1/2 cells, where actin filaments were repositioned into rosettes and lamellipodia were induced. Again, AFAP-110 ${ }^{\Delta z i p}$ colocalized with these structures. Identical results were noted in NIH3T3 fibroblasts, shown in panels G-I. These results indicate that AFAP-110 has the intrinsic ability to induce the reorganization of the actin cytoskeleton, establishing it as a potential effector for cellular signals which direct this action. 


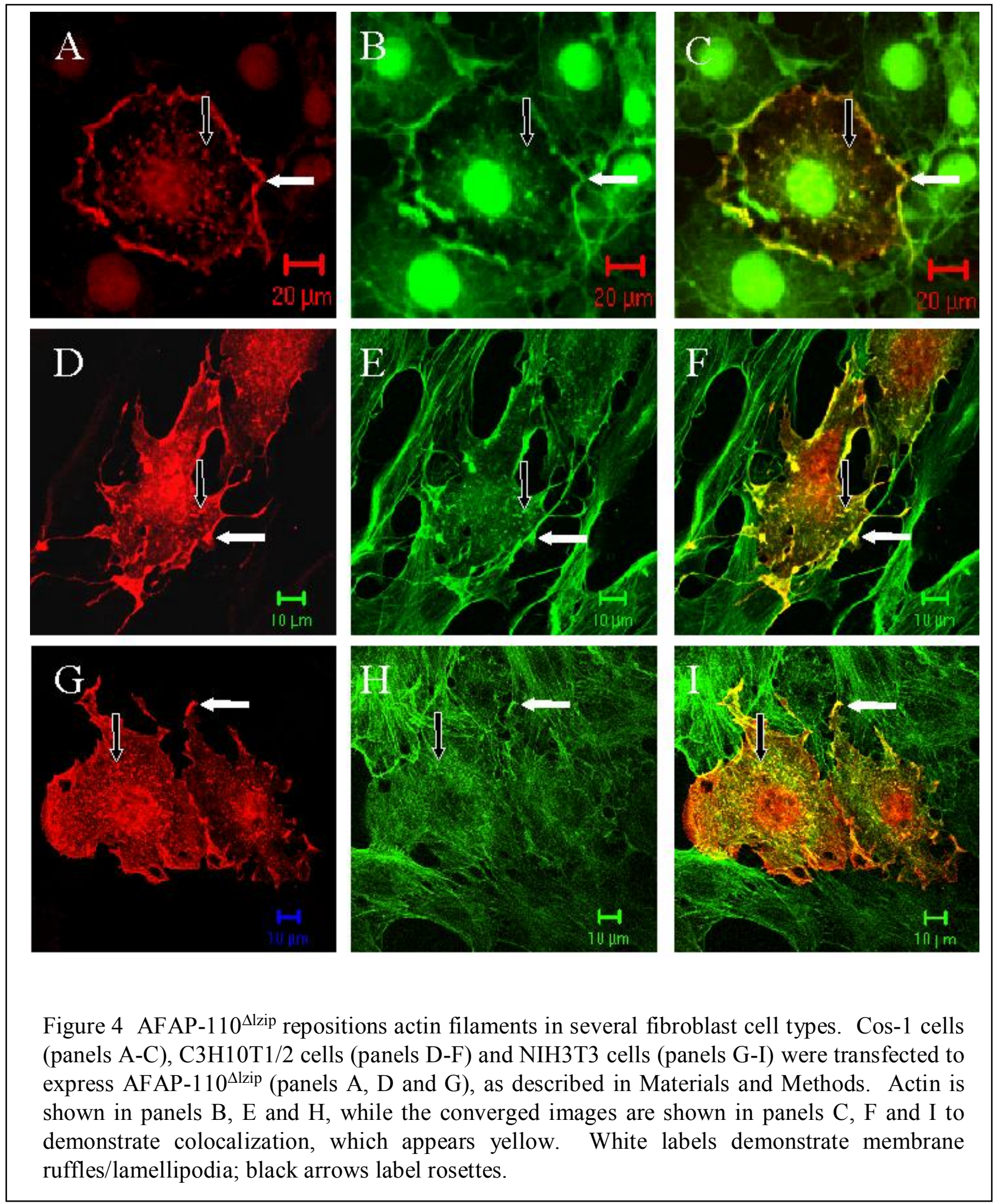




\section{Discussion}

The experiments reported here were designed to test the potential for AFAP-110 to bind actin structures directly and additionally confirm the potential for AFAP-110 to induce cytoskeletal rearrangements in other cell lines. Table 1 reveals that AFAP-110 contains two potential actin-binding motifs that overlap and extend from residue 601 to residue 615 . The sequence homology for the first of these domains, the ABD1, was lower than that seen in most other ABD1 domains presented. These domains shared a range of sequence similarities from 60\%-93\%, while this domain from AFAP-110 demonstrated a range of sequence homology of $33-47 \%$. This range was higher than that of the ABD1 from Tensin, which showed $20-47 \%$ homology with the other ABD1 regions. Thus, the ABD1 from AFAP-110 contained higher sequence homology to other ABD1 regions than at least one known functional ABD1, indicating this region may represent a member of this class of actin-binding domains. The second actinbinding domain, similar to an ABD3 motif, shares complete or nearly complete homology with other known domains of this type.

The result seen upon expression of AFAP- $110^{\Delta 114}$, depicted in Figure 2, panels J-L, allows us to address the contribution of both actin-binding domains toward interactions between AFAP-110 and actin. As seen in this figure, this mutant is deficient for colocalization with actin compared with wild-type AFAP-110, but does retain some colocalization with actin. This mutant, created by the engineering of a stop codon which truncated the protein 114 residues from the end, contains the entire ABD1. The protein ends at residue 615, which is the second residue of the ABD3. As this mutant maintains a complete ABD1 but an incomplete ABD3, it appears that both domains are involved in the interaction between AFAP-110 and actin. As these 
domains overlap, it seems likely that they would cooperate in binding actin, and the entire structure may therefore be required to maintain efficient interactions with actin.

The ability of AFAP-110 to bind actin directly was substantiated by Dr. Qian in a series of in vitro experiments which tested the ability of AFAP-110 to directly bind polymerized actin. These experiments demonstrated that residues 511-637 of AFAP-110 co-pellet with polymerized actin, indicating this region can bind actin directly (Qian et al., 2000). Additionally, it was confirmed that amino acids 593-637 of AFAP-110, containing both putative actin-binding domains, were the portion of this region responsible for the direct interaction with actin. Thus, it was shown that AFAP-110 binds actin directly via a region containing homology to known actinbinding domains.

One interesting result seen upon the expression of the AFAP-110 mutants presented here involves the localization of AFAP-110 to the cell membrane. Wild-type AFAP-110 appears to colocalize with the cell membrane, as seen in Figure 2, panels A-C. This colocalization is absent for the deletion mutants AFAP- $110^{\Delta 176}$ and AFAP-110 ${ }^{\Delta 136}$, as seen in Figure 2, panels D-F and G-I, respectively. Gray arrows in these panels highlight this result. Colocalization to the cell membrane is restored for the mutant AFAP-110 $0^{\Delta 114}$, indicating residues 616-637 are involved in the localization of AFAP-110 to the cell membrane. This colocalization may be direct or indirect, through the association with a binding partner located at the cell membrane. In fact, this result may simply reflect the association of AFAP-110 with actin structures at the cell membrane. Cells demonstrating AFAP-110 or mutants at the cell membrane consistently display actin structures at these sites. This is highlighted by AFAP- $110^{\Delta 114}$, which retains some colocalization with actin structures and also retains membrane localization, particularly with actin structures at the cell membrane. The expression of AFAP- $110^{\text {Slzip }}$ supports this idea as 
these cells show an increase in actin-rich membrane structures which are enriched for AFAP$110^{\Delta \text { lzip }}$.

The ability of AFAP-110 to bind actin directly, as shown here, combined with its ability to multimerize suggests AFAP-110 may function as an actin bundling or cross-linking protein. To better understand the implications of cross-linking, it is necessary to discuss actin dynamics. In eukaryotic cells, actin filaments (F-actin) are cross-linked into two types of structures by bundling proteins in vivo: these are the meshwork (isotropic network) and the bundle (Matsudaira, 1991; Wachsstock et al., 1993). The actin bundle is a structure where individual actin filaments are closely packed to form parallel arrays. This establishes similar polarity for the individual actin filaments, which have a growing (barbed) end and minus end. These structures are therefore thought to elongate as a unit and are thought to provide force against the cell membrane which results in the extension or propulsion of the membrane forward (Cramer, 1997; Matsudaira, 1991). This is a necessary step in cell migration. Actin filament bundles of this type mainly exist in two cell structures: a) membrane projections such as microvilli and cell motility organelles (filopodia, lamellipodia and membrane ruffles) and b) muscle sarcomeres. In constrast to actin bundles, the actin meshwork is a structure in which actin filaments form an orthogonally oriented network. This type of structure is found in the cell body and cell cortex (Matsudaira, 1991). Meshworks are thought, like bundles, to provide force against the cell membrane, similarly pushing the cell membrane (Pantaloni et al., 2001). In fact, bundle and network structures cooperate to form the actin-rich cell membrane structures which are necessary for cell migration, namely filopodia and lamellipodia/membrane ruffles (Cramer, 1997; Matsudaira, 1991). Often, filopodia extend from lamelliopodia, and where these structures mix, actin filaments exist as bundle and network structures that are the force that allow protrusion of 
filopodia and lamellipodia (Cramer, 1997; Matsudaira, 1991). In consideration of these types of structures, AFAP-110 seems a likely candidate for actin bundling. As shown in Table 1 and Figure 2, AFAP-110 contains two potential actin-binding domains. If these structures bind actin independently, AFAP-110 could bundle actin filaments in a monomeric form. However, the fact that these structures overlap suggests that they may cooperate to bind one actin filament. In support of this, AFAP-110 appears to multimerize in cells, suggesting that the monomeric form of AFAP-110 may be relatively rare and that AFAP-110 may bundle actin structures by dimerizing. Additionally, the multimerization of AFAP-110 is altered upon Src activation, indicating the bundling capacity of AFAP-110 may be altered. AFAP-110 therefore represents a potential actin cross-linking protein with a regulated ability to cross-link and may serve in the dynamic formation of filopodia and lamellipodia. The localization of AFAP-110 to actin-rich membrane structures upon Src activation (Qian et al., 1998) or deletion of the leucine zipper, as seen in Figure 4, supports this.

The ability of AFAP- $110^{\Delta \text { lzip }}$ to alter actin filaments combined with the ability of AFAP110 to directly bind actin filaments suggests that AFAP-110 may have a direct affect on actin filaments which is revealed upon deletion of the leucine zipper. This hypothesis has been examined by Dr. Yong Qian, and the results suggest that this is the case. This will be discussed in more detail in Chapter 4. Alternatively, deletion of the leucine zipper of AFAP-110 may result in the reorganization of the actin cytoskeleton by an indirect mechanism, such as the induction of cellular signals known to direct these rearrangements. Chapters 2 and 3 will address this second hypothesis. In reality, it appears that AFAP-110 may have both types of effects on actin structures, and Chapter 4 will attempt to reconcile these effects in a mechanistic study. 


\section{Materials and Methods}

Cell culture. Cos-1 cells were maintained and transfrected as previously described (Guappone and Flynn, 1997). C3H10T1/2 and NIH3T3 cells were grown as previously described (Luttrell et al., 1988). Transient transfections were carried out in all three cell lines as previously decribed (Qian et al., 1998). Specifically, calcium phosphate-mediated transfections were used for Cos-1 cells, while both C3H10T1/2 and NIH3T3 cells were transfected using SuperFect, as per manufacturer's instructions (Qiagen).

Generation of AFAP-110 mutants. The generation of all AFAP-110 mutants were carried out by Dr. Yong Qian in the following manner. AFAP- $110^{\Delta 176}$ and AFAP- $110^{\Delta l z i p}$ were generated as previously described (Qian et al., 1998). AFAP-110 $0^{\Delta 136}$ and AFAP-110 ${ }^{\Delta 114}$ were created using site-directed mutagenesis to introduce stop codons into the sequence of each of these mutants. Sequence analysis confirmed these stop codons, as well as the absence of spurious second site mutations. The predicted size of all mutants was confirmed via SDS-PAGE and western blot analysis with anti-AFAP-110 antibodies. GFP-tagged forms of AFAP-110 were created by cloning AFAP-110 into the eGFP-C3 vector (Clontech) using EcoRI, as originally linkered onto the chick AFAP-110 cDNA via PCR. PCR was also used to create EcoRI linkers surrounding the coding sequence for the Cterm peptide (residues 511-729), after which the product was inserted into the eGFP-C3 vector. The predicted size of these fusion proteins was confirmed by western blot analysis as well. 
Microscopic analysis. Immunofluorescence analysis was performed in the following manner. Cells were plated on coverslips and transfected at $>50 \%$ confluence to maintain a subconfluent culture at the time of fixation. At this time point, cells were washed with PBS and fixed with $3.7 \%$ formaldehyde for 30 minutes, followed by permeabalization with $0.1 \%$ Tx-100 for 4 minutes. In the case of cells expressing untagged forms of AFAP-110, labeling of AFAP-110 was accomplished using $\mathrm{mAb} 4 \mathrm{C} 3$ followed by rabbit anti-mouse conjugated to rhodamine (Sigma). FITC phalloidin (Sigma) was used to label actin filaments. In the case of cells expressing GFP-tagged forms of AFAP-110, BODIPY 650/655 phalloidin (Molecular Probes) was used to visualize actin filaments. Images were collected using a Zeiss LSM 510 microscope and scanned in grayscale. The images presented here were recolored to consistently display AFAP-110 as red and actin filaments as green. 


\section{References}

Cramer,LP. (1997). Front Biosci., 2, d260-d270.

Guappone,AC and Flynn,DC. (1997). Mol. Cell Biochem., 175, 243-252.

Luttrell,DK, Luttrell,LM, and Parsons,SJ. (1988). Mol. Cell Biol., 8, 497-501.

Matsudaira,P. (1991). Trends Biochem. Sci., 16, 87-92.

Pantaloni,D, Le Clainche,C, and Carlier,MF. (2001). Science, 292, 1502-1506.

Qian,Y, Baisden,JM, Westin,EH, Guappone,AC, Koay,TC, and Flynn,DC. (1998). Oncogene, 16, 2185-2195.

Qian,Y, Baisden,JM, Zot,HG, Van Winkle,WB, and Flynn,DC. (2000). Exp. Cell Res., 255, 102113.

Taylor,JM, Richardson,A, and Parsons,JT. (1998). Curr. Top. Microbiol. Immunol., 228, 135163.

Wachsstock,DH, Schwartz,WH, and Pollard,TD. (1993). Biophys. J., 65, 205-214. 


\section{Chapter 2}

The intrinsic ability of AFAP-110 to alter actin filament integrity is linked with its ability to also activate cellular tyrosine kinases. 
The actin filament-associated protein of $110 \mathrm{kDa}$ (AFAP-110) is a Src binding partner that represents a potential modulator of actin filament integrity in response to cellular signals. Previous reports have demonstrated that AFAP-110 is capable of directly binding and altering actin filaments. Deletion of the leucine zipper motif of AFAP-110 (AFAP-110 ${ }^{\Delta \mathrm{zzi}}$ ) has been shown to induce a phenotype which resembles Src-transformed cells, by repositioning actin filaments into rosettes. This deletion also mimics a conformational change in AFAP-110 that is detected in Src-transformed cells. The results presented here indicate that unlike AFAP-110, AFAP-110 ${ }^{\text {Alzip }}$ is capable of activating cellular tyrosine kinases, including Src family members, and that AFAP-110 ${ }^{\text {Uzip }}$ itself is hyperphosphorylated. The newly tyrosine-phosphorylated protein and activated Srcfamily members appear to be associated with actin-rich lamellipodia. A point mutation that alters the SH3-binding motif of AFAP-110 ${ }^{\text {Alzip }}$ prevents it from activating tyrosine kinases and altering actin filament integrity. In addition, a deletion within a pleckstrin homology (PH) domain of AFAP-110 ${ }^{\text {Azip }}$ will also revert its effects upon actin filaments. Lastly, dominant-positive RhoA ${ }^{\mathrm{V} 14}$ will block the ability of AFAP-110 ${ }^{\text {Uzip }}$ from inducing actin filament rosettes. Thus, conformational changes in AFAP-110 enable it to activate cellular kinases in a mechanism requiring $\mathrm{SH} 3$ and/or $\mathrm{PH}$ domain interactions. We hypothesize that cellular signals which alter AFAP-110 conformation enable it to activate cellular kinases, which then direct changes in actin filament integrity in a Rho-dependent fashion. 


\section{Introduction}

Activation of the Src oncogene is associated with characteristic changes in the actin cytoskeleton of cultured cells (Felice et al., 1990; Hamaguchi and Hanafusa, 1987; Reynolds et al., 1989). These changes include a repositioning of actin filaments into rosette-like structures, and the formation of lamellipodia and filopodia, which are associated with increased cell motility and the onset of tumor metastasis in humans (Bolen et al., 1987; Boschek et al., 1981; Cartwright et al., 1990; Irby et al., 1999; Rosen et al., 1986; Tarone et al., 1985). The cSrc proto-oncogene can be activated by dephosphorylation of $\mathrm{Tyr}^{527}$ by cellular phosphatases, or displacement of repressive, intramolecular interactions involving the $\mathrm{SH} 2$ and $\mathrm{SH} 3$ domains (Brown and Cooper, 1996). These activation events normally occur in response to cellular signals, e.g., growth factors interacting with their receptors (Brown and Cooper, 1996). These pathways are thought to proceed through Src, with the subsequent phosphorylation of substrates and activation of downstream signaling members, including Ras (He et al., 2000), pp125 ${ }^{\text {FAK }}$ (Thomas et al., 1998), Crk (Sabe et al., 1992) and pp130 ${ }^{\text {Cas }}$ (Xing et al., 2000).

The actin filament associated protein, AFAP-110, is a tyrosine-phosphorylated substrate of Src and an SH2/SH3 binding partner for $\operatorname{Src}^{527 F}$ (Flynn et al., 1993). AFAP-110 is also capable of being an SH2/SH3 binding partner for cFyn and cLyn (Flynn et al., 1993; Guappone and Flynn, 1997). AFAP-110 appears to function as an adaptor molecule, linking other proteins to the actin cytoskeleton, potentially in multi-protein complexes. AFAP-110 contains several putative protein binding domains, including $\mathrm{SH} 2$ and $\mathrm{SH} 3$ binding motifs, two pleckstrin homology (PH) domains, and a leucine zipper (Flynn et al., 1993). Additionally, AFAP-110 contains a carboxy terminal, actin-binding domain (Qian et al., 2000). These data indicate that 
AFAP-110 could function by serving to link Src, as well as a variety of other signaling proteins, to actin filaments.

The presence of a leucine zipper motif indicated a mechanism by which AFAP-110 could self-associate (Qian et al., 1998). Cellular AFAP-110 can be affinity absorbed from cell lysates with GST-encoded fusion proteins expressing the leucine zipper motif; however, co-expression of $\mathrm{Src}^{527 \mathrm{~F}}$ abrogated the ability of these fusion proteins to absorb cellular AFAP-110. Gel filtration analysis confirmed that AFAP-110 does exist in a self-associated, multimeric complex as tetramers, trimers and also monomers and that the leucine zipper was necessary for multimerization (Flynn et al., 2001; Qian et al., 1998). Co-expression of $\mathrm{Src}^{527 \mathrm{~F}}$ reduces the size of self-associated AFAP-110 complexes to a single population hypothesized to represent dimers (Qian et al., 1998). Because there appeared to be a change in conformation of AFAP-110 in response to $\mathrm{Src}^{527 \mathrm{~F}}$ signaling that affected the leucine zipper motif, this same motif was deleted (AFAP-1 $10^{\Delta \text { lzip }}$. The deletion construct was expressed in cells to determine what the effect of loss of function of the leucine zipper motif may have upon AFAP-110 in cells. Biochemically, deletion of the leucine zipper motif enabled AFAP-110 ${ }^{\text {Alzip }}$ to exist as a dimer, based upon gel filtration analysis (Qian et al., 1998). In cells, expression of AFAP-110 $0^{\Delta l z i p}$ affected a significant change in cell morphology and actin filament integrity, repositioning actin filaments into rosettelike structures, not unlike those seen in Src-transformed cells, and inducing lamellipodia formation (Qian et al., 1998; Qian et al., 2000). Thus, AFAP-110 has an intrinsic capability to alter actin filament integrity, which may be revealed by cellular signals that direct changes in its conformation.

In this work, we hypothesized that AFAP-110 $10^{\Delta l z i p}$ directed changes in actin filament integrity either directly or indirectly. A direct mechanism was defined as one in which AFAP- 
110 alters actin filament integrity by virtue of an ability to physically bind and remodel actin filaments. An indirect mechanism was defined as one in which AFAP-110 activated cellular signaling pathways known to modulate actin filament integrity. The results shown here indicate that AFAP- $110^{\Delta \text { lzip }}$ induces the activation of cellular tyrosine kinases, including Src-family members, thus repositioning actin filaments into rosettes via an indirect mechanism. These events are blocked by two separate genetic alterations in AFAP-110; one a mutation in the SH3binding motif, AFAP- $110^{\mathrm{P} 71 \mathrm{~A} / \Delta \mathrm{Lzip}}$, the second a deletion in an amino-terminal pleckstrin homology $(\mathrm{PH})$ domain, AFAP-110 $1180-226 / \Delta$ zip , both of which show no increased levels of tyrosine phosphorylation or Src family activation and neither of which effected actin filament integrity. Co-expression of constitutively active Rho $\left(\mathrm{RhoA}^{\mathrm{V} 14}\right)$ also overcomes the ability of AFAP- $110^{\Delta l z i p}$ to alter actin filaments, indicating the effects of AFAP- $110^{\Delta l z i p}$ to generate actin rosettes and lamellipodia are indirect. Herein, we hypothesize that AFAP-110 has the potential to alter actin filaments in response to cellular signals that would alter its conformation, resulting in activation of cellular kinases via its ability to interact with SH3-containing and PH domainbinding partners. 


\section{Results}

Deletion of the leucine zipper motif enables AFAP-110 to alter actin filament integrity

The expression of AFAP- $110^{\Delta l z i p}$ results in a cell phenotype which resembles Src-transformed cells, while overexpression of wild-type AFAP-110 has no effect on actin structures (Qian et al., 1998; Qian et al., 2000). Cells expressing AFAP-110 $0^{\text {Alzip }}$ consistently displayed actin-rich rosettes in place of actin filaments, as well as actin-rich lamellipodia. Filopodia are also detected in some of these cells. Figure 1 demonstrates that GFP-tagged AFAP-110 expressed in C3H10T1/2 cells co-localizes with actin filaments and the cell membrane (panels A and B), while GFP-AFAP- $110^{\Delta \text { szip }}$ co-localizes with and induces the formation of actin filament-rich rosettes (white arrowheads, panels $\mathrm{C}$ and $\mathrm{D}$ ) and lamellipodia (gray arrowheads, panels $\mathrm{C}$ and D). Lamellipodia were noted as actin-rich structures at the cell membrane of variable size. GFP expressed alone in these cells will not co-localize with actin filaments or affect cytoskeletal structures (Qian et al., 2000). Similar results were also detected in Cos-1 fibroblasts (data not shown). These data indicate that the leucine zipper plays a regulatory role for AFAP-110 and indicate that AFAP-110 also has an intrinsic ability to reposition actin filaments into rosettes and generate lamellipodia formation when the leucine zipper is no longer present. 


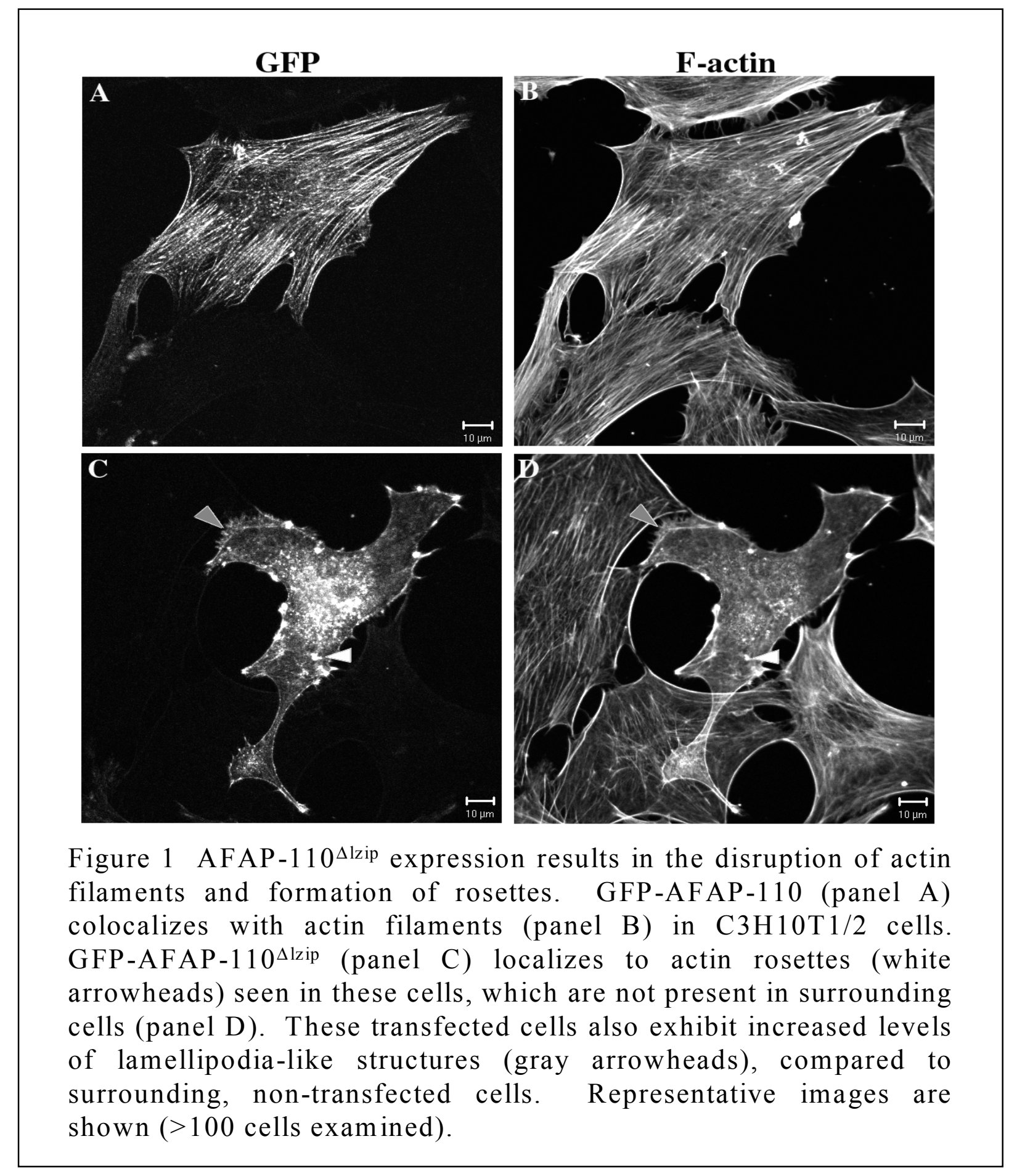


Expression of AFAP-110 $0^{\Delta l z i p}$ directs an increase in cellular tyrosine phosphorylation

As AFAP-1 $10^{\Delta l z i p}$ induces a phenotype which resembles Src activation, we sought to determine if this mutant could alter actin filaments by activating cellular kinases. Phosphoamino acid analysis was performed on AFAP-110 and AFAP-110 ${ }^{\text {Alzip }}$ from transfected Cos-1 cells. Figure $2 \mathrm{~A}$ indicates that under equal loading conditions, over-expressed AFAP- $110^{\Delta \text { lzip }}$ contains elevated levels of serine, threonine, and tyrosine phosphorylation, compared to over-expressed wild-type AFAP-110. Phosphorylation was increased by 1.6 fold, 2.6 fold and 2.4 fold, respectively, as determined by scanning densitometry. Anti-phosphotyrosine antibodies were used for western blot analysis of immunoprecipitated proteins to confirm the increase in tyrosine phosphorylation levels of AFAP-110 ${ }^{\Delta l z i p}$ relative to AFAP-110 (Figure 2B). Also, GFP-tagged forms of AFAP-110 demonstrated levels of phosphotyrosine similar to their untagged counterparts (data not shown). These data indicate that AFAP-110 $0^{\Delta l z i p}$ becomes hyperphosphorylated on serine, threonine, and tyrosine residues, relative to wild-type AFAP110. 
A
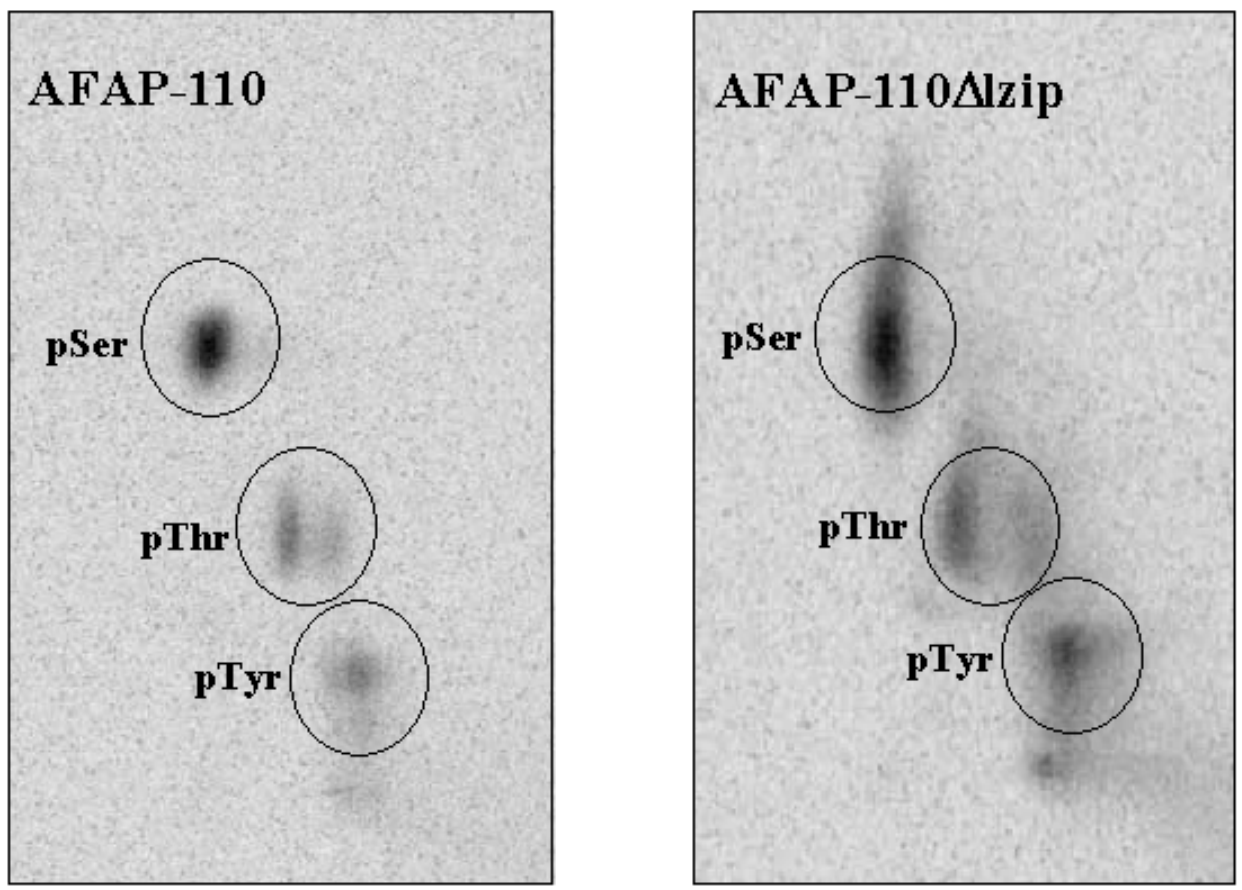

B

\section{Phosphotyrosine}

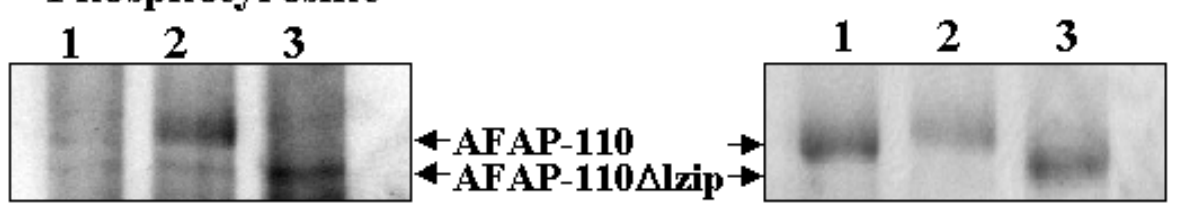

Figure 2 AFAP-110 $10^{\Delta \text { zip }}$ is hyperphosphorylated upon expression in Cos-1 cells. (A) Phosphoamino acid analysis (Boyle et al., 1991) of radio-labeled AFAP-110 and AFAP-110 1 lzip immunoprecipitated from transfected Cos-1 cells (as in Figure 1) demonstrates that AFAP-110 1 lzip (right panel) is hyperphosphorylated compared to AFAP-110 (left panel). ImageQuant analysis of spot intensity revealed 1.6 fold, 2.6 fold and 2.4 fold increases in serine, threonine, and tyrosine phosphorylation, respectively. Western blot analysis of mAb 4C3 immunoprecipitations followed by rabbit anti-phosphotyrosine immunoblotting AFAP-110 (lane 1), AFAP110 co-expressed with $\mathrm{Src}^{527 \mathrm{~F}}$ (lane 2) and AFAP-110 $0^{\text {szip }}$ (lane 3) from Cos-1 cell lysate. (C) The same western blot from B was stripped and reprobed with $\mathrm{mAb} 4 \mathrm{C} 3$ to demonstrate protein loading levels. Lane: 1AFAP-110, 2- AFAP-110/Src ${ }^{527 F}, 3-$ AFAP-110 ${ }^{\Delta \text { lzip }}$. The western blot shown is representative of three independent experiments. 
AFAP-110 $0^{\Delta l z i p}$ expression activates cellular tyrosine phosphorylation

The increases in tyrosine phosphorylation of AFAP- $110^{\Delta l z i p}$ indicated that it may induce the activation of cellular kinases. Anti-phosphotyrosine antibodies were used to determine if total cellular tyrosine phosphorylation was increased and to which subcellular sites tyrosine substrates were localized in these cells. Figure 3 demonstrates the results obtained using immunofluorescence of transiently transfected C3H10T1/2 cells. Anti-phosphotyrosine immuno-labeling revealed no significant change in phosphotyrosine levels in GFP-AFAP-110 transfected cells; however, an overall increase in tyrosine phosphorylation throughout the cell, as well as a co-localization of phosphotyrosine-containing proteins with GFP-AFAP-110 1 llzip and actin-rich structures at the cell membrane, was evident in GFP-AFAP- $110^{\Delta \text { lzip }}$ transfected cells (white arrowheads, panels D-F). Similar results were seen in Cos-1 cells (data not shown). It is noteworthy that some labeling of tyrosine-phosphorylated proteins with the anti-phosphotyrosine antibody was apparent along the cell membrane and in actin-rich structures in non-transfected cells; however, the intensity of immunolabeling was significantly lower than AFAP- $110^{\text {Alzip }}$ expressing cells. These structures appeared to represent lamellipodia, which are known to contain tyrosine-phosphorylated proteins and whose formation and extension involve Src activation (Boschek et al., 1981; Schwartzberg et al., 1997). The levels of immunostaining for these tyrosine-phosphorylated proteins were also noticeably lower in cells expressing wild-type AFAP-110, which was equivalent to untransfecteded cells (Figure 3 A-C). These results demonstrate an increase in cellular tyrosine-phosphorylated substrates along the cell membrane and within actin-rich structures in response to the expression of GFP-AFAP- $110^{\text {Alzip }}$. 


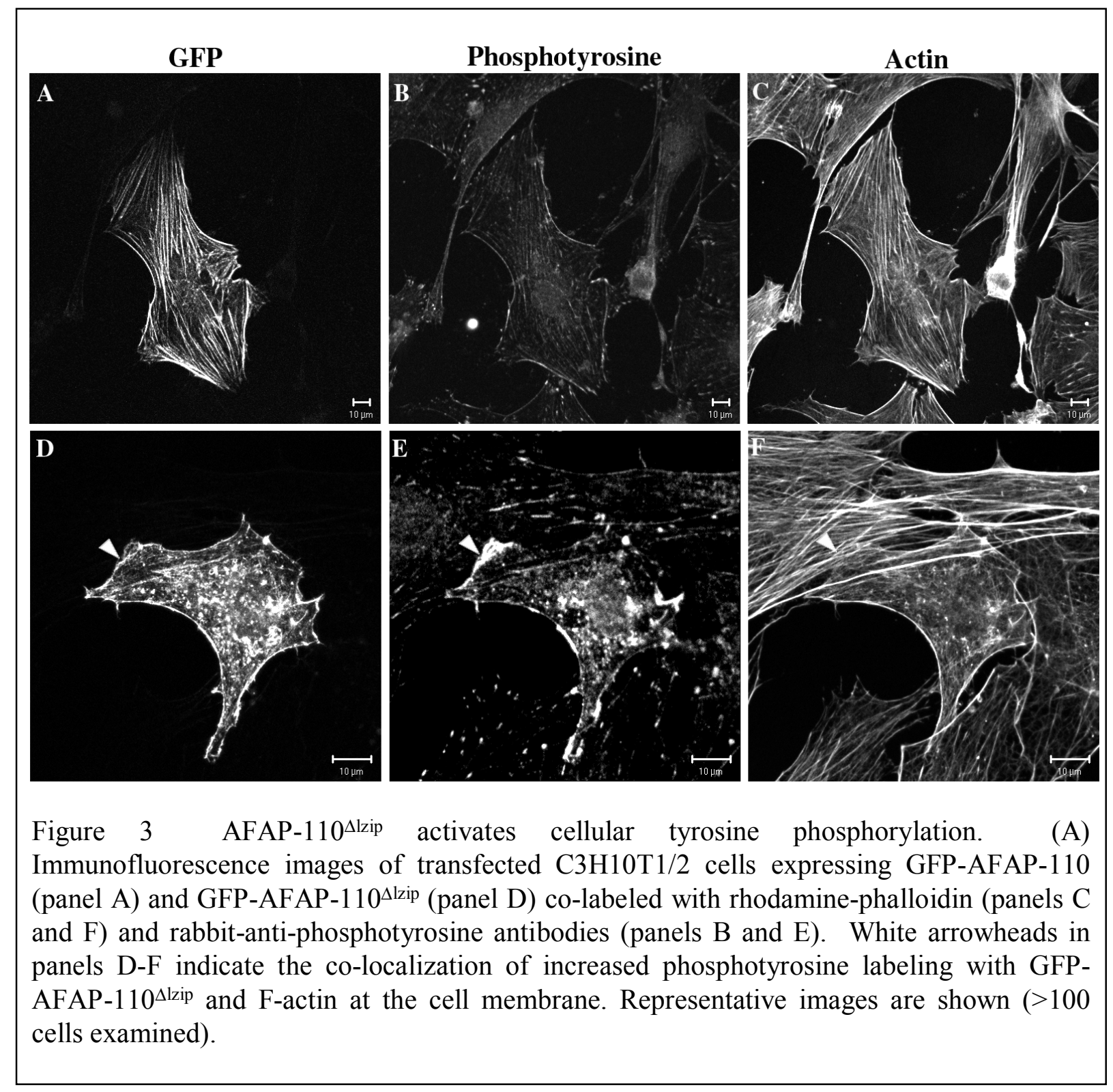


AFAP-110 $0^{\Delta l z i p}$ expression results in the specific activation of Src family kinases

Specific Src family activation-state antibodies were used to determine if AFAP- $110^{\Delta l z i p}$ affected autophosphorylation of Src family kinases, an indicator for activation of these tyrosine kinases. Immunofluorescence analysis of $\mathrm{C} 3 \mathrm{H} 10 \mathrm{~T} 1 / 2$ cells expressing GFP-AFAP-110 revealed no significant increase in immunoreactivity compared to untransfected cells, as shown in Figure 4 (Panels A-C). However, co-localization of these activated Src family members with GFPAFAP- $110^{\Delta \text { zzip }}$ and actin-rich structures at the cell membrane was evident (white arrowheads, panels D-F). These cells also displayed an increase in fluorescence intensity throughout the cell for the anti-Src activation state antibody, compared to surrounding, non-transfected cells. This result correlated well with GFP-AFAP-110 ${ }^{\Delta l z i p}$ expression levels. Similar results were seen in Cos-1 cells, in which the colocalization of GFP-AFAP-110 1 Lzip with actin and activated Src occurred at membrane ruffles and lamellipodia-like structures (data not shown). Like the antiphosphotyrosine antibodies shown in Figure 3, this Src family activation-state antibody showed some labeling of protein in non-transfected cells, mostly in what appears to be perinuclear structures. These results indicate that expression of GFP-AFAP-110 $0^{\Delta l z i p}$ can direct autophosphorylation of one or more Src family kinases throughout the cell and specifically in actin-rich structures that co-localize with GFP-AFAP-110 1 lzip along the cell membrane. 


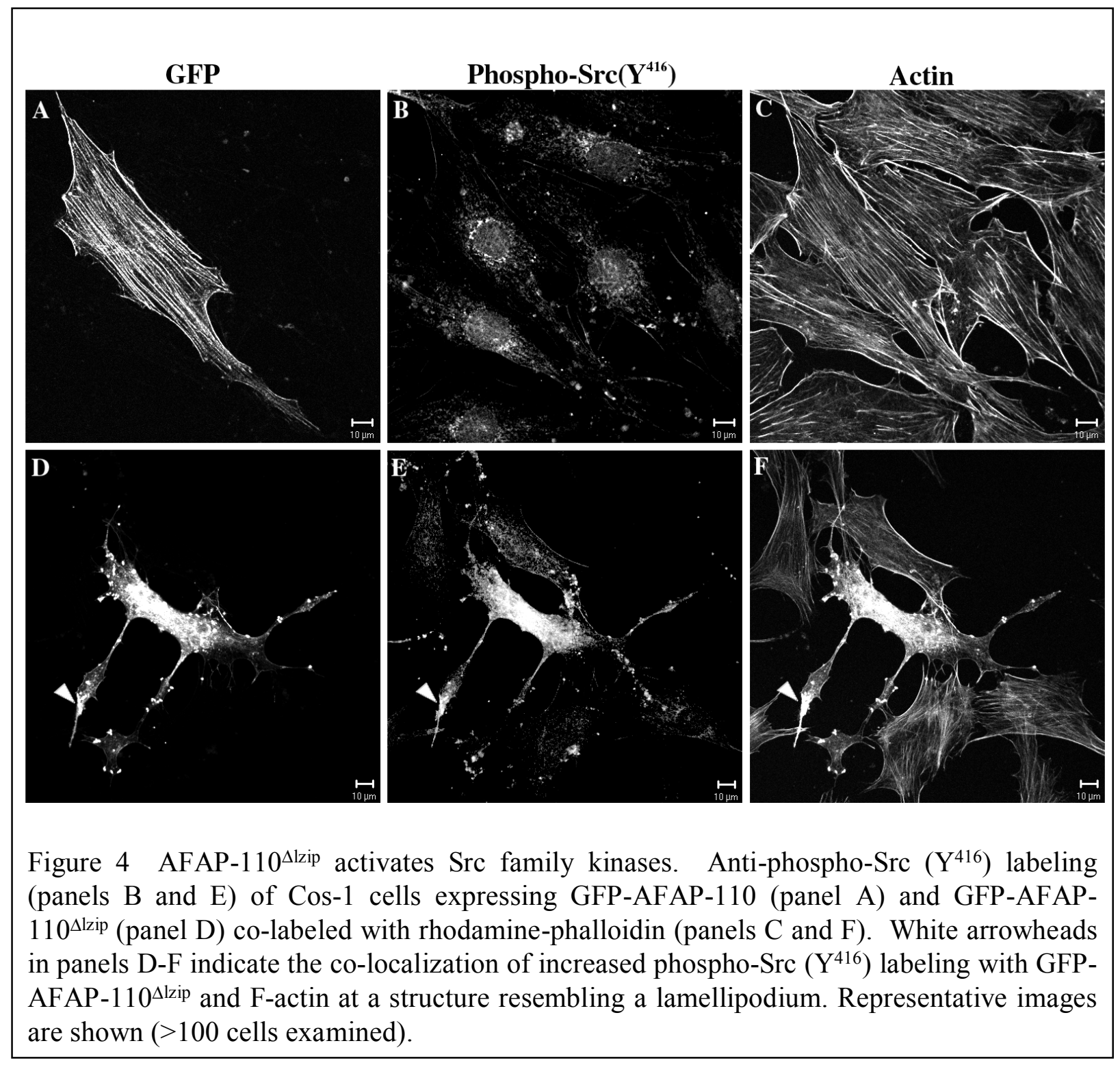


A mutation which alters the SH3-binding motif in AFAP-110 prevents AFAP-110 ${ }^{\Delta l z i p}$ from activating tyrosine kinases or altering actin filament integrity.

Because some SH3-binding partners can activate Src-family kinases via SH3 binding (Moarefi et al., 1997), it was hypothesized that AFAP- $110^{\Delta l z i p}$ may be able to activate Src-family kinases in a similar manner, as AFAP-110 is also an SH3-binding partner for cSrc, cFyn or cLyn (Flynn et al., 1993); (Guappone and Flynn, 1997). To test this, AFAP-110 $0^{\mathrm{P} 1 \mathrm{~A} / \Delta \mathrm{\Delta zip}}$ was created, which contains an additional point mutation in the SH3-binding motif that has been shown to abrogate SH3 interactions with Src, and also prevents Src/AFAP-110 stable complex formation (Guappone and Flynn, 1997). AFAP-110 $0^{\mathrm{P} 1 \mathrm{~A} / \Delta \mathrm{Azip}}$ was expressed in C3H10T1/2 cells to examine the ability of this mutant to activate Src family kinases. Figure 5 demonstrates that this mutant failed to induce an increase in cellular tyrosine phosphorylation (panels A-C). Additionally, AFAP- $110^{\mathrm{P} 71 \mathrm{~A} / \Delta \mathrm{Lzip}}$ failed to increase cellular immunoreactivity with Src family kinase activationstate antibodies (panels D-F). These cells displayed no increase in labeling by these phosphorylation-state antibodies, compared to non-transfected surrounding cells, unlike those expressing GFP-AFAP-110 ${ }^{\text {Lzip }}$ (Figure 3). AFAP- $110^{\text {P71A/Alzip }}$ also failed to alter actin filaments or induce lamellipodia formation, while maintaining co-localization with actin filaments. Thus, SH3 domain binding by AFAP-110 $10^{\Delta l z i p}$ appears to be necessary for its ability to activate cellular tyrosine phosphorylation, Src family kinases and cytoskeletal rearrangements. 


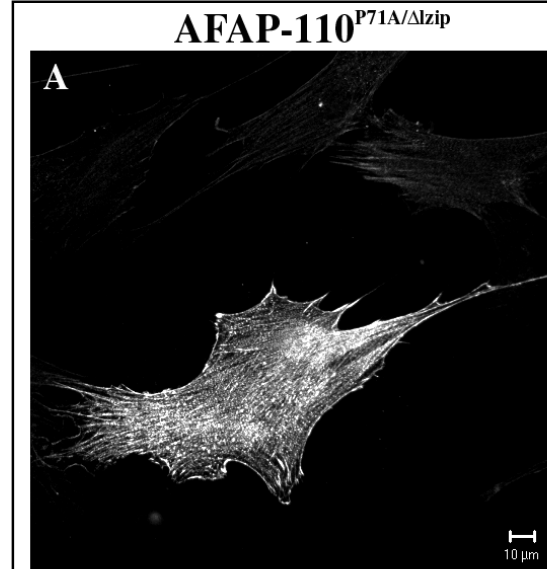

AFAP-110 ${ }^{\text {P71A/Alzip }}$

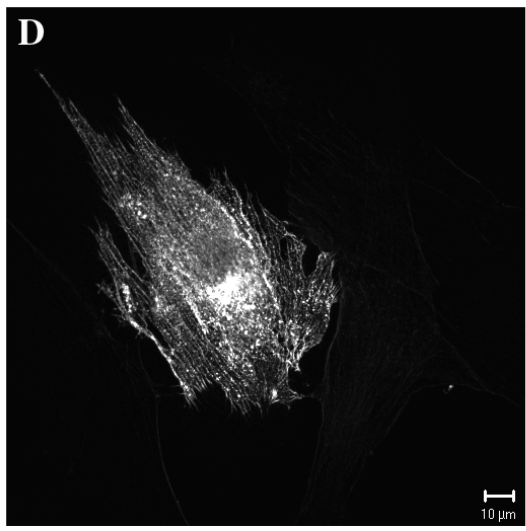

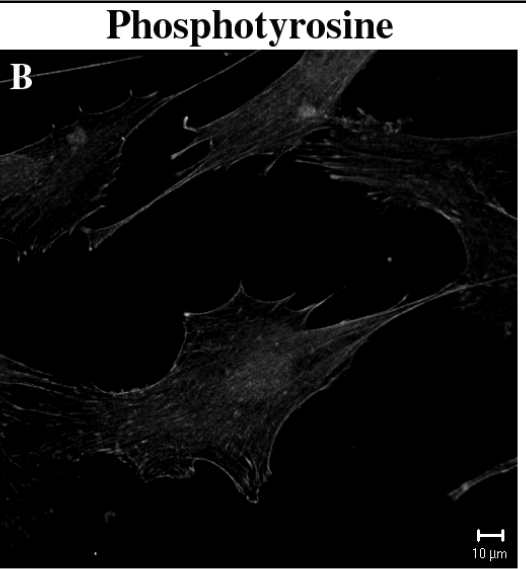

Phospho-Src $\left(\mathbf{Y}^{416}\right)$

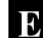

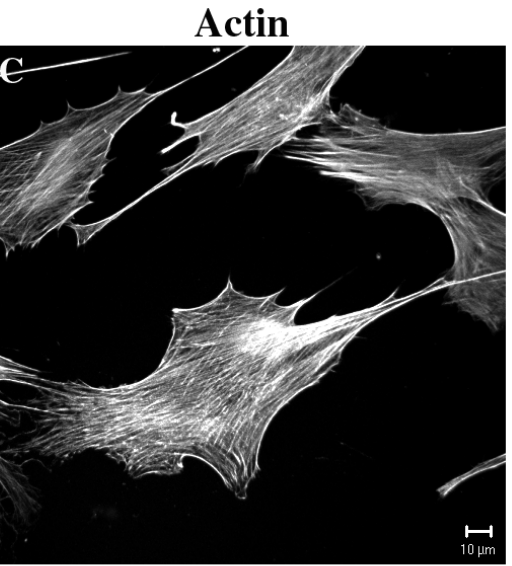

Actin

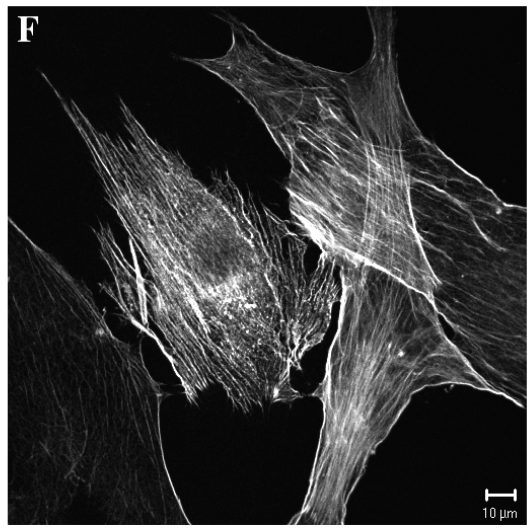

Figure 5 Abrogation of SH3 binding inhibits the cytoskeletal rearrangement and activation

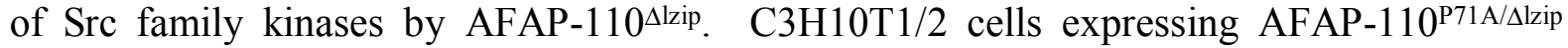
(panels A and D) were labeled as above with anti-phosphotyrosine (panel B) and antiphospho-Src $\left(\mathrm{Y}^{416}\right)$ (panel E) antibodies. Panels $\mathrm{C}$ and $\mathrm{F}$ show normal actin filaments, labeled with rhodamine-phalloidin. ( $>100$ cells examined). 
The integrity of the PH domain of AFAP-110 is also required for AFAP-110 ${ }^{\Delta l z i p}$-directed increases in cellular tyrosine phosphorylation, Src family activation and cytoskeletal rearrangements

GFP-AFAP- $110^{\Delta 180-226 / \Delta / z i p}$ contains an additional deletion of 46 amino acids in the amino-terminal PH domain, which is predicted to disrupt the function of this domain. Figure 6 demonstrates the results obtained upon labeling C3H10T1/2 cells expressing GFP-AFAP$110^{\Delta 180-226 / \Delta l z i p}$ with both anti-phosphotyrosine (panels A-C) and Src family activation state antibodies (panels D-F). This mutant fails to induce cytoskeletal rearrangements, as actin filaments (panels C and F) are abundant and the mutant (panels A and D) colocalizes with these structures. Additionally, this mutant fails to induce cellular tyrosine hyperphosphorylation (panel B) or Src family kinase activation (panel E), compared to surrounding, non-transfected cells. Also, AFAP-110 ${ }^{\Delta 180-226 / \Delta l z i p}$ had reduced levels of phosphotyrosine compared to AFAP$110^{\Delta l z i p}$ (data not shown). Similar results were seen in Cos-1 cells. Thus, the amino-terminal PH domain of AFAP-110 also plays an important role in the ability of AFAP-110 ${ }^{\text {Slzip }}$ to activate Src and alter actin filament integrity. 


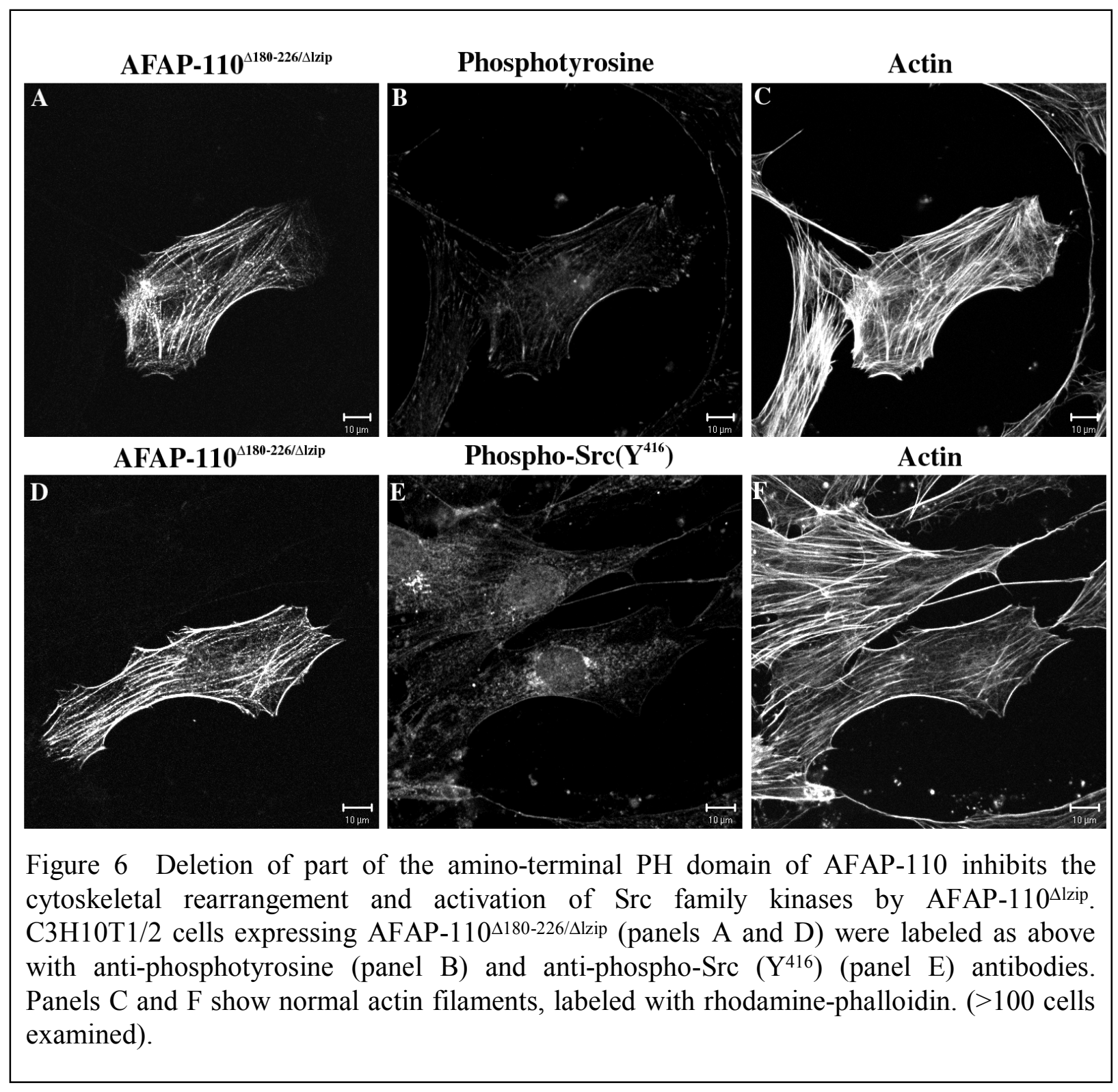


AFAP-110 $0^{\Delta l z i p}$ alters actin filaments in a Rho-dependent fashion

The GTP-binding protein, Rho, plays an important role in effecting changes in actin filaments downstream of Src. Activated Rho can restore actin filament organization in Srctransformed cells (Mayer et al., 1999), and cSrc activation requires the inactivation of Rho to induce actin filament dissolution (Fincham et al., 1999). GFP-AFAP-110 1 szip was co-expressed in $\mathrm{C} 3 \mathrm{H} 10 \mathrm{~T} 1 / 2$ cells with dominant positive Rho $\left(\mathrm{RhoA}^{\mathrm{V} 14}\right)$ to determine if GFP-AFAP-110 1 lzip may exert its affects on actin filaments in a Rho-dependent fashion. Figure 7 shows that cells expressing AFAP-110 and RhoA ${ }^{\mathrm{V} 14}$ were consistently filled with well-developed actin filaments (panels A-C). Similar results were seen in Cos-1 cells (data not shown). Interestingly, in GFPAFAP-110 ${ }^{\text {Llzip }}$-expressing cells, RhoA ${ }^{\mathrm{V} 14}$ overcomes the ability of GFP-AFAP- $110^{\Delta \text { Lzip }}$ to reposition actin filaments into rosettes (panels D-F), consistent with previous results that demonstrated significantly increased amounts of F-actin (Ridley and Hall, 1992). It is noteworthy that GFP-AFAP- $110^{\Delta l z i p}$ is more prominently represented along the cell membrane and within small membrane protrusions, while less well associated with stress filaments, compared to AFAP-110 co-expressed with RhoA ${ }^{\mathrm{V} 14}$. These results demonstrate that the ability of AFAP- $110^{\Delta l z i p}$ to reposition actin filaments into rosettes occurs in a Rho-dependent fashion, and, like Src, may require the down-regulation of Rho to complete this function. Thus, the repositioning of actin filaments into rosettes and the induction of lamellipodia formation by AFAP-110 $10^{\Delta l z i p}$ may proceed largely through indirect mechanisms that permit it to activate Srcfamily kinases and the subsequent activation of downstream signals which ultimately alter actin filament organization. 


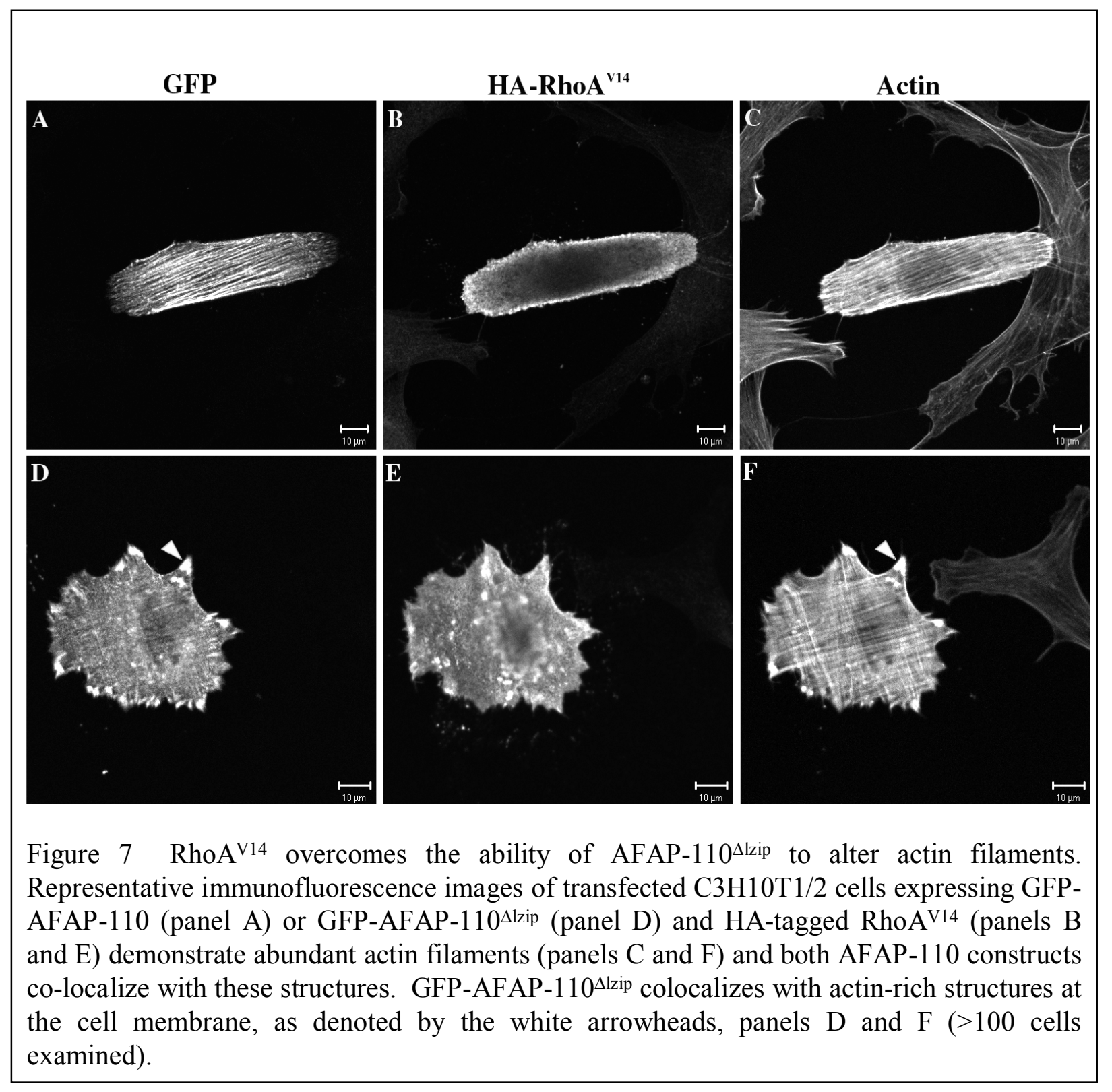




\section{Discussion}

The actin-binding protein, Src binding partner and substrate AFAP-110 has been proposed to function as a mediator of cytoskeletal rearrangements in response to Src activation (Flynn et al., 1993; Kanner et al., 1991). AFAP-110 receives cellular signals, as it is hyperphosphorylated on serine, threonine, and tyrosine residues in Src-transformed cells (Kanner et al., 1991). Previous reports have also shown that AFAP-110 binds F-actin structures directly, indicating that it could also directly affect actin filament integrity (Qian et al., 2000). Additional studies demonstrated that AFAP-110 self-associates via a mechanism involving a carboxy-terminal leucine zipper motif, indicating the potential for AFAP-110 to cross-link actin filaments (Qian et al., 1998). Co-expression of $\mathrm{Src}^{527 \mathrm{~F}}$ will induce a conformational change in AFAP-110 that abrogates selfassociations through the leucine zipper motif. Thus, AFAP- $110^{\Delta l z i p}$ represents a model system or approximation of the conformation of AFAP-110 as detected in $\mathrm{Src}^{527 \mathrm{~F}}$-transformed cells. Interestingly, signals generated by $\mathrm{Src}^{527 \mathrm{~F}}$ that affect the conformation of AFAP-110 appear to be the result of serine/threonine phosphorylation, rather than tyrosine phosphorylation (Qian et al., 1998). The changes in conformation that abrogate self-association through the leucine zipper will also occur in response to PMA treatment and activation of PKC, while inhibitors of PKC block these changes in conformation in response to $\mathrm{Src}^{527 \mathrm{~F}}$ or PMA (Baisden et al., in preparation). These data indicate that cellular signals generated downstream of $\mathrm{Src}^{527 \mathrm{~F}}$, possibly involving PKC, will enable a conformational change that reduces the ability of AFAP-110 to multimerize via its leucine zipper motif. It is noteworthy that deletion of the leucine zipper motif also enables AFAP- $110^{\Delta \text { lzip }}$ to reposition actin filaments into rosettes, not unlike the effects of $\mathrm{Src}^{527 \mathrm{~F}}$ or PMA treatment on cells. Thus, conformational changes that affect AFAP-110 in 
response to cellular signals may enable it to direct changes in actin filament organization. However, because AFAP-110 both binds actin filaments directly and is a binding partner for signaling proteins, it was unclear whether these changes in actin filament integrity were the result of direct or indirect effects of AFAP- $110^{\Delta \text { lzip }}$ upon the actin-based cytoskeleton. The data generated here demonstrate that AFAP- $110^{\text {slzip }}$ can direct upregulation of cellular tyrosine phosphorylation and the activation state of Src-family kinases, based on immunoreactivity in these cells using phosphorylation-specific antibodies. In addition, the changes in actin filament integrity that result in actin rosette formation occur in a Rho-dependent fashion, indicating that AFAP-110 ${ }^{\text {Llzip }}$ can effect actin filament, rosette and lamellipodia formation by an indirect mechanism involving activation of cellular signaling cascades known to control actin filament dynamics.

One possible mechanism by which AFAP- $110^{\Delta \text { lzip }}$ could activate tyrosine kinases like cSrc is through its ability to function as an SH2/SH3 binding partner (Flynn et al., 1993; Guappone et al., 1998; Guappone and Flynn, 1997; Kanner et al., 1991; Reynolds et al., 1989). It has been shown the SH3 binding partners, such as the HIV encoded gene product, Nef, can activate the Src family member Hck via SH3 binding (Briggs et al., 1997; Moarefi et al., 1997). It has also been demonstrated that intramolecular interactions that keep Src or Hck in the repressed conformation involve the SH3 domain binding to a left-handed, polyproline type II helix in the linker domain (Liu and Pawson, 1994). Nef, by virtue of its ability to function as an SH3 binding partner for Hck, is able to displace the intramolecular SH3 interaction of Hck and subsequently direct activation of the kinase. Similarly, it is possible that within the conformation represented by AFAP-110 $0^{\text {lzip }}$, AFAP-110 becomes a more favorable SH3-binding partner for Src and may activate Src-family members by a similar mechanism. This hypothesis is supported 
by the results which indicate that $\mathrm{SH} 3$ interactions are necessary for AFAP- $110^{\Delta l z i p}$ to activate Src family kinases and induce cytoskeletal rearrangements (Figure 5). Additionally, the ability of RhoA ${ }^{\mathrm{V} 14}$ to block the ability of AFAP-110 $10^{\Delta l z i p}$ to rearrange the actin cytoskeleton (Figure 7) indicates that the actions of AFAP-110 $10^{\Delta l z i p}$ on the cytoskeleton are a result of activating signaling cascades through Src to Rho family GTPases.

The abrogation of hyperphosphorylation and the cytoskeletal reorganization induced in cells expressing AFAP- $110^{\text {Slzip }}$ by the additional deletion in the PH domain indicates that this domain may also play a significant role in the action of AFAP-110 toward the actin cytoskeleton. It is possible that this loss of function occurs due to the interruption of interactions with one or more potential $\mathrm{PH}$ domain binding partners, rather than due to a change in subcellular localization. This interpretation is based on the observation that GFP-AFAP-110 $10^{\Delta 180-226 / \Delta l z i p}$ is detected on the cell membrane, similar to wild-type AFAP-110. The amino terminal PH domain is also capable of affinity absorbing several binding partners from cellular lysate, including PKC (Baisden et al., in preparation). This interaction is abrogated by deletion of residues 180-226, thus, it is possible that this $\mathrm{PH}$ domain deletion may abrogate interactions with a putative binding partner necessary for the activation of Src normally seen upon deletion of the leucine zipper of AFAP-110. Alternatively, a PH domain deletion variant may fail to permit AFAP-110 to approximate the subcellular localization of $\mathrm{cSrc}$, or other kinases, and this may prevent activation of tyrosine phosphorylation.

Interestingly, RhoA ${ }^{\mathrm{V} 14}$ was able to block the ability of AFAP-110 ${ }^{\Delta l z i p}$ from inducing actin filament rosette formation. However, in $\mathrm{RhoA}^{\mathrm{V} 14}$-expressing cells, we noted differences in the subcellular localization of AFAP-110 compared to AFAP-110 ${ }^{\text {Szip }}$. AFAP-110 was evenly associated with stress filaments and the cell membrane. In AFAP- $110^{\text {Ulzip }}$-expressing cells, this 
mutant protein was very strongly associated with actin-rich cell membrane protrusions when coexpressed with RhoA ${ }^{\mathrm{V} 14}$, and less associated with stress filaments. Recent data from our lab indicated that AFAP-110 ${ }^{\Delta \text { zzip }}$ does exert direct effects upon actin filaments by increasing actin filament cross-linking, in vitro (Qian et al., in preparation). It may be possible that AFAP$110^{\Delta l z i p}$ also has an ability to effect actin filament cross-linking as a consequence of direct interactions and, thus, this capability could be amplified in the presence of RhoA ${ }^{\mathrm{V} 14}$.

In this report, we demonstrate a potential mechanism by which AFAP-110 ${ }^{\Delta l z i p}$ can mediate changes in actin filament integrity by activation of cellular tyrosine kinases. These results indicate that AFAP- $110^{\text {slzip }}$ repositions actin filaments into rosettes by an indirect mechanism which activates cellular kinases in an SH3-binding and PH-binding dependent fashion. We predict that upstream cellular signals can alter the conformation of AFAP-110, enabling it to activate cellular tyrosine kinases, such as Src-family kinases. The nature of the upstream cellular signals that effect AFAP-110 may involve activation of PKC family members. Unpublished data from our lab demonstrate that AFAP-110 is a substrate for PKC and that changes in conformation detected within AFAP-110 in response to $\mathrm{Src}^{527 \mathrm{~F}}$ signaling occur in a PKC-dependent fashion (Baisden et al., in preparation). These conformational changes appear to effect the multimeric status of AFAP-110 and may enable it to present itself as a more favorable SH3-binding partner to Src-family kinases, such as cSrc or cFyn, resulting in activation of these signaling cascades and the concomitant changes in actin filament integrity. Ultimately, activation of this signaling cascade may direct activation of Rho-family GTPases which exert their effects upon the actin-based cytoskeleton. 


\section{Materials \& Methods}

Reagents

Dulbecco's modified Eagle's medium, Basal Medium Eagle, and Rhodamine-phalloidin were obtained from Sigma. AFAP-110 antibodies 4C3 and F1 were generated and characterized as previously described (Qian, et al., 1999). Anti-phosphotyrosine was purchased from BD Transduction Labs. Anti-phospho-Src $\left(\mathrm{Y}^{416}\right)$ was purchased from Cell Signaling (Massachusetts). Anti-rabbit Alexa 633 and anti-mouse Alexa 488 were obtained from Molecular Probes. Anti-HA tag antibody was purchased from Santa Cruz Biotechnology.

\section{Cell Culture}

Cos-1 cells were maintained and transfected as previously described (Guappone and Flynn, 1997). C3H10T1/2 cells were cultured as previously described (Qian et al., 2000). Transient transfections of C3H10T1/2 cells were carried out using Effectene (Qiagen) as per manufacturers instructions. Transient transfections of Cos-1 cells employed the CalPhos transfection kit (Clontech).

\section{Phosphoamino acid analysis}

Cos- 1 cells were grown to $60 \%$ confluence in $100 \mathrm{~mm}$ culture dishes, and transiently transfected. After 48 hours, the cells were serum starved overnight. Cells were washed twice with PBS and 
lysed with $1 \mathrm{ml}$ RIPA buffer, as previously described (Qian et al., 1998). One and one-half $\mu 1$ monoclonal antibody 4C3 ascites was used to immunoprecipitate AFAP-110 from the lysate, which was isolated via SDS-PAGE. The radioactive band was excised from the gel and subjected to partial acid hydrolysis and phosphoamino acid analysis (Boyle et al., 1991). After running the isolated amino acids on 2D TLC, the plates were imaged using a Phosphorimager (Molecular Dynamics). Spots were identified by running labeled phosphoserine, phosphothreonine and phosphotyrosine markers. Relative intensity compared to background of radiation from spots was quantitated with ImageQuant software.

\section{Immunofluorescence}

C3H10T1/2 cells were transiently transfected, as above. 48 hours after transfections, the cells were serum-starved overnight. Cells were fixed and permeabalized as previously described (Qian et al., 1998). After washing, cells were labeled for 30 minutes. For actin labeling, a 1:1000 dilution of rhodamine-phalloidin was used. Antibody concentrations used: 4C3, $0.6 \mu \mathrm{g} / \mathrm{ml}$ in $5 \% \mathrm{BSA}$; Anti-HA, $4 \mu \mathrm{g} / \mathrm{ml}$ in $5 \% \mathrm{BSA}$; anti-phosphotyrosine, $2.5 \mu \mathrm{g} / \mathrm{ml}$ in $5 \% \mathrm{BSA}$; anti-phospho-Src $\left(\mathrm{Y}^{416}\right)$ labeling, $5 \mu \mathrm{l} / \mathrm{ml}$ in 5\%BSA. Both polyclonal phospho-specific antibodies were visualized by incubating the cells for 30 minutes with anti-rabbit-Alexa 633 $(5 \mu \mathrm{l} / \mathrm{ml}$ in $5 \% \mathrm{BSA})$ after washing off primary antibody. Monoclonal antibody $4 \mathrm{C} 3$ was visualized using Alexa 488 anti-mouse secondary antibody $(5 \mu \mathrm{l} / \mathrm{ml}$ in $5 \% \mathrm{BSA})$. Cells were washed and mounted on slides with Fluoromount (Fisher). A Zeiss LSM 510 microscope was used to gather grayscale images, which represent confocal slices of about $2 \mu \mathrm{M}$ in thickness. Scale bars were generated and inserted by LSM 510 software. 


\section{References}

Bolen,JB, Veillette,A, Schwartz,AM, DeSeau,V, and Rosen,N. (1987). Proc. Natl. Acad. Sci. U. S. $A, \mathbf{8 4}, 2251-2255$.

Boschek,CB, Jockusch,BM, Friis,RR, Back,R, Grundmann,E, and Bauer,H. (1981). Cell, 24, 175-184.

Boyle,WJ, van der,GP, and Hunter,T. (1991). Methods Enzymol., 201, 110-149.

Briggs,SD, Sharkey,M, Stevenson,M, and Smithgall,TE. (1997). J Biol. Chem., 272, 1789917902.

Brown,MT and Cooper,JA. (1996). Biochim. Biophys. Acta, 1287, 121-149.

Cartwright,CA, Meisler,AI, and Eckhart,W. (1990). Proc. Natl. Acad. Sci. U. S. A, 87, 558-562.

Felice,GR, Eason,P, Nermut,MV, and Kellie,S. (1990). Eur. J. Cell Biol., 52, 47-59.

Fincham,VJ, Chudleigh,A, and Frame,MC. (1999). J Cell Sci., 112 ( Pt 6), 947-956.

Flynn,DC, Leu,TH, Reynolds,AB, and Parsons,JT. (1993). Mol. Cell Biol., 13, 7892-7900.

Flynn,DC, Qian,Y, and Baisden,JM. (2001). Oncogene,

Guappone,AC and Flynn,DC. (1997). Mol. Cell Biochem., 175, 243-252.

Guappone,AC, Weimer,T, and Flynn,DC. (1998). Mol. Carcinog., 22, 110-119.

Hamaguchi,M and Hanafusa,H. (1987). Proc. Natl. Acad. Sci. U. S. A, 84, 2312-2316.

He,H, Hirokawa,Y, Levitzki,A, and Maruta,H. (2000). Cancer J, 6, 243-248.

Irby,RB, Mao,W, Coppola,D, Kang,J, Loubeau,JM, Trudeau,W, Karl,R, Fujita,DJ, Jove,R, and Yeatman,TJ. (1999). Nat. Genet., 21, 187-190.

Kanner,SB, Reynolds,AB, and Parsons,JT. (1989). J. Immunol. Methods, 120, 115-124.

Kanner,SB, Reynolds,AB, Wang,HC, Vines,RR, and Parsons,JT. (1991). EMBO J., 10, 16891698.

Liu,X and Pawson,T. (1994). Recent Prog. Horm. Res., 49, 149-160.

Mayer,T, Meyer,M, Janning,A, Schiedel,AC, and Barnekow,A. (1999). Oncogene, 18, $2117-$ 2128.

Moarefi,I, LaFevre-Bernt,M, Sicheri,F, Huse,M, Lee,CH, Kuriyan,J, and Miller,WT. (1997). Nature, 385, 650-653. 
Qian,Y, Baisden,JM, Westin,EH, Guappone,AC, Koay,TC, and Flynn,DC. (1998). Oncogene, 16, 2185-2195.

Qian,Y, Baisden,JM, Zot,HG, Van Winkle,WB, and Flynn,DC. (2000). Exp. Cell Res., 255, 102113.

Reynolds,AB, Kanner,SB, Wang,HC, and Parsons,JT. (1989). Mol. Cell Biol., 9, 3951-3958.

Ridley,AJ and Hall,A. (1992). Cell, 70, 389-399.

Rosen,N, Bolen,JB, Schwartz,AM, Cohen,P, DeSeau,V, and Israel,MA. (1986). J. Biol. Chem., 261, 13754-13759.

Sabe,H, Okada,M, Nakagawa,H, and Hanafusa,H. (1992). Mol. Cell Biol., 12, 4706-4713.

Schwartzberg,PL, Xing,L, Hoffmann,O, Lowell,CA, Garrett,L, Boyce,BF, and Varmus,HE. (1997). Genes Dev., 11, 2835-2844.

Tarone,G, Cirillo,D, Giancotti,FG, Comoglio,PM, and Marchisio,PC. (1985). Exp. Cell Res., 159, 141-157.

Thomas,JW, Ellis,B, Boerner,RJ, Knight,WB, White,GC, and Schaller,MD. (1998). J Biol. Chem., 273, 577-583.

Xing,L, Ge,C, Zeltser,R, Maskevitch,G, Mayer,BJ, and Alexandropoulos,K. (2000). Mol. Cell Biol., 20, 7363-7377. 
Chapter 3

AFAP-110 activates Src through SH3 interactions in response to PKC phosphorylation 
The Src oncogene directs the transformation of cells to a metastatic phenotype via directed reorganization of the actin cytoskeleton. This reorganization can be stimulated by extracellular signals including growth factor stimulation, which activates Src via receptor activity. Other signaling components, like PKC, direct similar rearrangements of the actin cytoskeleton in response to other extracellular signals. Here we provide evidence for a mechanism of activation of $\mathrm{Src}$ in response to $\mathrm{PKC}$ activation. This mechanism requires AFAP-110, a Src binding partner/substrate, previously shown to undergo a conformational change in response to Src activation. Phosphorylation of AFAP-110 by PKC drives this conformational change, which is shown to result in the activation of Sre in vitro via $\mathrm{SH} 3$ interactions. Expression of active PKC is shown to activate Src in vivo, as well, and AFAP110 mutants deficient for interactions with either PKC or Src block this event. These results provide an alternative mechanism whereby the activation of Src can be potentiated, in a feedback-type mechanism by PKC, or uniquely induced in the absence of stimuli traditionally thought to direct Sre activation. 


\section{Introduction}

Growth factor receptors utilize intracellular signaling cascades of proteins including Src and PKC to direct the normal growth and maintenance of tissues. These pathways often become deregulated in the case of uncontrolled cancerous growth, with tumors stimulating themselves in an autocrine fashion (Baserga et al., 1994; Kim and Muller, 1999; Lazar-Molnar et al., 2000). Other cases of uncontrolled growth show less evidence of external stimulation by the normal growth factor pathway and are often thought to involve the mutations of the proteins in the signaling cascades involved in controlling cell growth (Deman and Van Larebeke, 2001; LazarMolnar et al., 2000; Loeb, 2001). Either case results in the transformation of cells characterized by reorganization of the cytoskeleton and increased potential for cell migration resulting in metastasis. The signaling pathways that have been investigated to understand this cytoskeletal reorganization have revealed roles for Src and PKC. It has been shown that both Src and PKC activation can result in a similar transformed phenotype, yet no common mechanism for cytoskeletal reorganization has been found. Additionally, little evidence for the mutationinduced activation of either Src or PKC has been found, yet both are hyperactive in many tumors, indicating these proteins may be activated by deregulated cellular signals (Bolen et al., 1987; Cartwright et al., 1989; Cartwright et al., 1990; Delage et al., 1993; Rosen et al., 1986).

One protein which may provide a consensual mechanism for both Src- and PKCmediated cytoskeletal rearrangements is the actin filament-associated protein of $110 \mathrm{kDa}$, AFAP110. AFAP-110 was originally identified as a SH2/SH3 binding partner for Src that co-localized with actin filaments as described previously. AFAP-110 binds actin filaments directly and has the potential for forming signaling complexes on both cytoskeletal and membranous structures. It had been previously reported that AFAP-110 has direct affects on actin filament structural 
integrity, perhaps through a mechanism involving actin bundling, as AFAP-110 can selfassociate via a carboxy-terminal leucine zipper (Qian submitted). This work indicated that deletion of this leucine zipper resulted in a cell phenotype which resembled Src transformation. Chapter 2 also addressed the potential for this mutant to have indirect affects on the actin cytoskeleton and showed that expression of this mutant resulted in the activation of Src family kinases. This activation was shown to be dependent upon the integrity of both the SH3 motif and amino-terminal PH domain of AFAP-110. Another work has described AFAP-110 as a PKC substrate, both in vitro and in vivo (Qian submitted). Here, we further address the potential for AFAP-110 to have indirect affects on cytoskeletal structures as we examine the requirement of both the SH3 motif and PH domain for cytoskeletal reorganization. The data indicate that AFAP-110 interacts directly with PKC via the amino-terminal PH domain, and that phosphorylation of AFAP-110 by PKC induces a conformational change in the protein. This conformational change mimics that seen upon co-expression with active Src (Qian et al., 1998) or upon deletion of the leucine zipper (Flynn, unpublished data). Additionally, this conformational change results in the direct activation of Src in vitro in an SH3-dependent mechanism. Expression of active PKC results in the activation of Src in vivo, as well, which is dependent upon the integrity of both the SH3 motif and PH domain of AFAP-110. These results establish a crucial role for AFAP-110 in the activation of Src via a novel pathway involving PKC. 


\section{Results}

The amino-terminal PH domain of AFAP-110 directs interactions between AFAP-110 and PKC.

AFAP- $110^{\Delta \text { lzip }}$ expression can activate Src and this ability is dependent upon the integrity of the amino-terminal PH domain of AFAP-110. As AFAP-110 is hyperphosphorylated on serine and threonine residues in addition to tyrosine residues in Src-transformed cells (Kanner et al., 1991) and PH domains are thought to facilitate interactions with ser/thr kinases (Rodriguez et al., 1999; Yao et al., 1997), we hypothesized that this PH domain directs interactions with ser/thr kinases necessary for the activation of Src. To test this hypothesis, we addressed the potential for the $\mathrm{PH}$ domains to interact with ser/thr kinases. Figure 1A indicates the results of a colorimetric kinase assay using fusion proteins representing both PH domains of AFAP-110. The absorbates from both PH domains appeared to contain ser/thr kinase activity, although the amino-terminal PH domain appeared to absorb a kinase that more efficiently phosphorylated the substrate. Sequence analysis of both PH domains revealed highest homology between the amino-terminal PH domain and PH domains from B-spectrin and Dynamin, which have been shown to forge interactions with PKC (Baraldi et al., 1999; Rodriguez et al., 1999; Yao et al., 1994; Yao et al., 1997). The carboxy-terminal PH domain was found to share highest homology with the PH domain from Btk, which also directs interactions with PKC (Yao et al., 1994). To determine whether either $\mathrm{PH}$ domain from AFAP-110 could direct interactions with PKC, affinity absorptions from chick brain lysate using fusion proteins representing both $\mathrm{PH}$ domains were analyzed by SDS-PAGE. Figure 1B demonstrates by western blot analysis that 4 distinct proteins were detected using a pan-PKC antibody. In this experiment, only GST-PH1, 
A
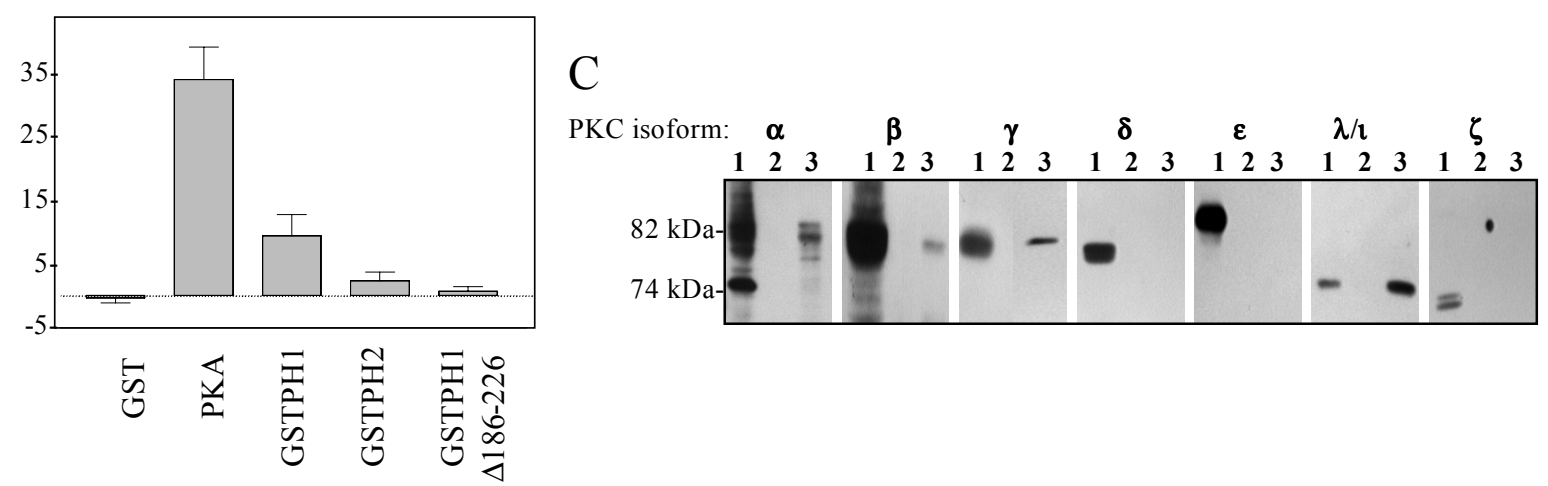

B

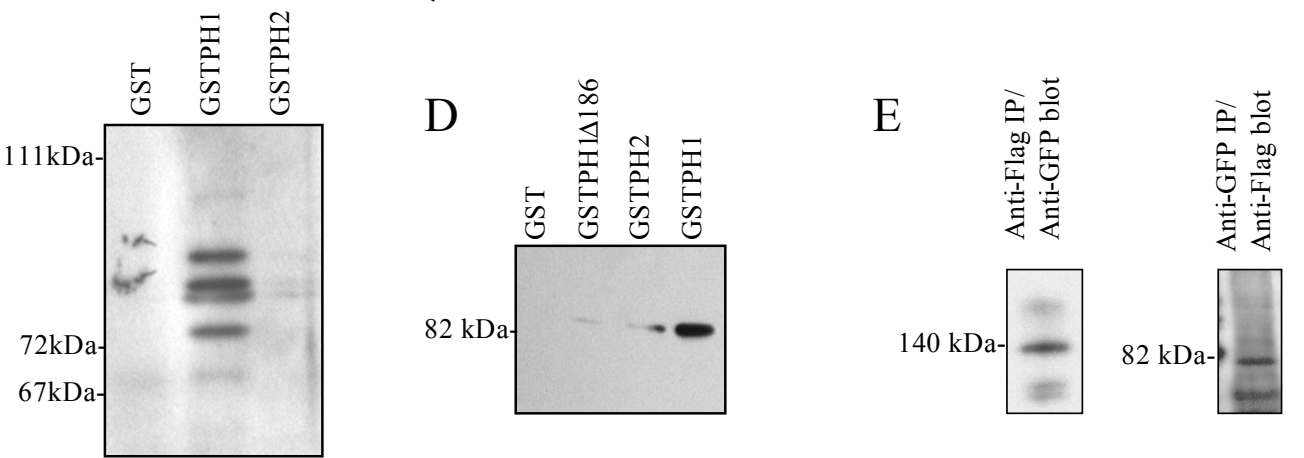

Figure 1 The amino-terminal $\mathrm{PH}$ domain of AFAP-110 promotes direct interactions between AFAP-110 and PKC isoforms. (A) The PH domains of AFAP-110 affinity absorb serine/threonine kinase activity. Affinity absorptions were performed as described in Materials and Methods using fusion proteins representing GST alone or GST fused to either the aminoterminal or carboxy-terminal PH domain of AFAP-110, labeled GST-PH1 and GST-PH2, respectively. GST-PH1 $\Delta 186-226$ represents a deletion mutant of the amino-terminal PH domain, as described in the results section. Absorbates were subjected to a colorimetric PKA assay, and compared to recombinant PKA. Values were normalized relative to negative control. Error bars represent standard deviation. N=3 for GST, PKA, and GST-PH1. N=2 for GST-PH2 and GST-PH1 $186-226$. (B) GST-PH1 affinity absorbs $\alpha$ pan-PKC-reactive proteins. Affinity absorptions were performed as above. Absorbates were analyzed by SDS-PAGE and western blotted with a pan-PKC antibody. (C) GST-PH1 affinity abosorbs conventional PKC isoforms and PKC $\lambda$. Absorbates from affinity absorptions as above were analyzed by SDS-PAGE and western blotted with isoform specific PKC antibodies, as listed. Lanes for each isoform blot are as follows: lane 1- lysate reference, lane 2- GST, lane 3- GST-PH1. Each blot is representative of at least two experiments. (D) GST-PH1 binds PKC $\alpha$ directly. Fusion proteins were used to affinity absorb recombinant $\mathrm{PKC} \alpha$, as described in Materials and Methods. Absobates were analyzed by SDS-PAGE and western blotted with a monoclonal antibody specific for PKC $\alpha$. This blot is representative of 3 experiments. (E) AFAP-110 and PKC co-immunoprecipitate. Immunoprecipitations were performed as described in Materials and Methods. Anti-GFP immunoprecipitation from lysate of Cos-7 cells co-expressing GFP-AFAP-110 and Flag-tagged, wild-type PKC $\alpha$ was analyzed by SDS-PAGE and probed with anti-Flag antibodies, as labeled. Similarly, anti-Flag immunoprecipitation from similar cell lysate was analyzed by SDS-PAGE and probed with anti-GFP antibodies. 
representing the amino-terminal PH domain, was able to efficiently affinity absorb PKCimmuno-reactive proteins. To determine which isoforms of PKC the GST-PH1 fusion protein could affinity absorb, additional affinity absorptions and western blots were processed using antibodies specific for PKC isoforms. Figure $1 \mathrm{C}$ shows that antibodies specific for PKC $\alpha, \beta, \gamma$, and $\lambda / \mathrm{l}$ isoforms demonstrate the presence of bands in the absorbate from the amino-terminal PH domain. Significantly, the absorbate was devoid of bands immunoreactive with antibodies against $\mathrm{PKC} \delta, \varepsilon$, and $\zeta$. Thus, the amino-terminal PH domain of AFAP-110 exhibits specificity for at least $4 \mathrm{PKC}$ isoforms. To determine if this interaction could be direct, purified recombinant PKC $\alpha$ was used in affinity absorptions with GST-PH1 domain fusion proteins. An additional fusion protein was used in this study which consisted of a deletion mutant of the amino-terminal PH domain. To create this mutant, a second Spe I restriction site was engineered into the cDNA encoding the GST-PH1 fusion protein by site-directed mutagenesis. This new site allowed for the deletion of amino acids 180-226, as designated in the wild type protein, resulting in a new fusion protein termed GST-PH1 1 180-226. This region was targeted as it contains homology to other $\mathrm{PH}$ domains thought to bind $\mathrm{PKC}$ by this $\mathrm{PH}$ domain region (Yao et al., 1997). Figure 1D shows that GST-PH1 is able to absorb PKC $\alpha$ with much higher affinity than GST-PH2 domain, the GST-PH1 $\Delta 180-226$ mutant, or the GST protein alone. A separate experiment showed that the GST-PH1 $180-226$ mutant also fails to affinity absorb PKC-reactive bands from chick brain lysate (data not shown). These results indicate that the amino-terminal PH domain of AFAP-110 has the potential to form a direct interaction with the PKC $\alpha, \beta, \gamma$, and $\lambda / \mathrm{t}$ isoforms.

To confirm that AFAP-110 and PKC interact in vivo, co-immunoprecipitation experiments were employed. Figure 1E shows that Flag-tagged PKC $\alpha$ co-expressed with GFP- 
tagged AFAP-110 can be co-immunoprecipitated using anti-GFP antibodies. Likewise, GFPtagged AFAP-110 can be co-immunoprecipitated with anti-Flag antibodies. These results confirm that AFAP-110 and PKC interact in vivo in a mechanism involving the amino-terminal PH domain of AFAP-110.

Abrogation of PH/PKC interactions blocks the hyperphosphorylation of AFAP-110 by PKC.

The mutant used in Chapter 2 to demonstrate that the ability of AFAP- $110^{\text {Alzip }}$ to activate Src was dependent upon the PH domain, AFAP-110 $1180-226$, was used here to study the effects of this deletion on the phosphorylation of AFAP-110 by PKC. Here, AFAP-110 ${ }^{\Delta 180-226}$ was purified from cell lysate and subjected to phosphamino acid analysis. The results are presented in Figure 2 and indicate that this deletion decreases the incorporation of radioactive phosphate into this mutant on serine, threonine and tyrosine residues, in response to PMA treatment, which activates PKC. The incorporation of radioactivity is compared to that for wild-type AFAP-110, similarly treated. Compared to wild-type, AFAP- $110^{\Delta 180-226}$ showed decreases of 0.55 fold, 0.49 fold, and 0.55 fold for serine, threonine and tyrosine phosphorylation, respectively. Thus, deletion of residues 180-226 from the amino-terminal $\mathrm{PH}$ domain of AFAP-110 impedes the hyperphosphorylation of AFAP-110 in vivo in response to PKC activation. 


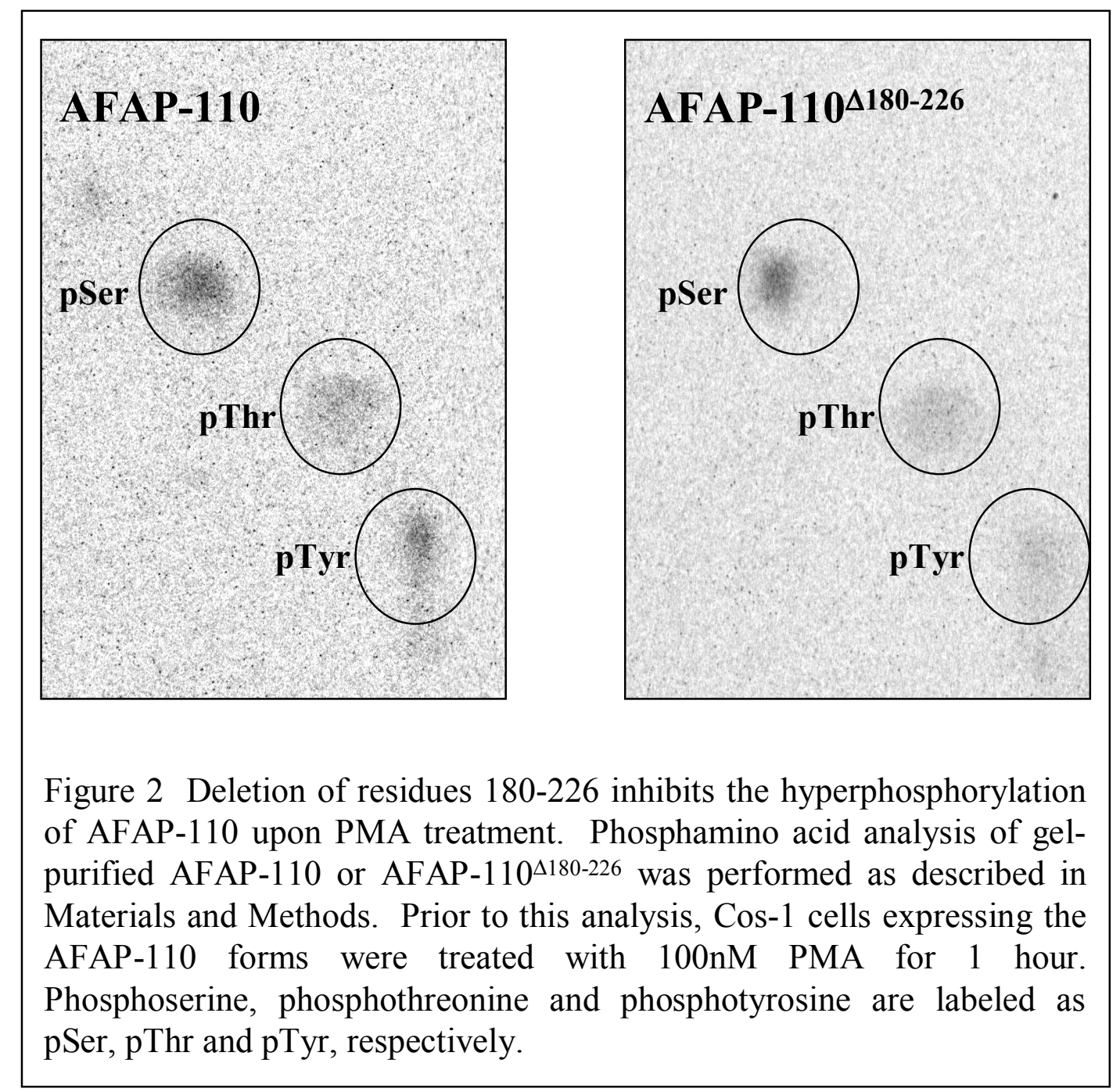

It is interesting that this deletion reduces the hyperphosphorylation of AFAP-110 on tyrosine in addition to serine and threonine residues. This could indicate that AFAP-110 may require interactions with $\mathrm{PKC}$, and potentially the hyperphosphorylation by $\mathrm{PKC}$, in order to be presented as a tyrosine substrate. This would imply that tyrosine kinases, including Src family kinases, may be activated here. Alternatively, this result could simply indicate the inhibition of the activation of tyrosine kinases in these cells. This would be consistent with the results presented in Chapter 2, which indicate that the activation of Src family kinases by AFAP-110 1 lzip is inhibited by this deletion of residues $180-226$. 
Serine/threonine phosphorylation of AFAP-110 alters the ability of AFAP-110 to self-associate.

Previous work has shown that AFAP-110 is hyperphosphorylated on serine and threonine residues in Src-transformed cells and that this hyperphosphorylation correlates with a conformational change in AFAP-110 (Qian et al., 1998). This conformational change is measured by a change in the self-association of AFAP-110. A work in submission has established AFAP-110 as a potential PKC substrate (Qian submitted). Here, we sought to determine if PKC phosphorylation directs this conformational change. Figure 2 indicates that the integrity of the $\mathrm{PH}$ domain is required for the hyperphosphorylation of AFAP-110 upon the activation of PKC. This figure includes the results of a phosphamino acid analysis comparing the incorporation of radioactive phosphate into AFAP-110 and AFAP-110 $\Delta 180-226$ from Cos-1 cells treated with $100 \mathrm{nM}$ PMA for 1 hour after serum starvation overnight. Comparison of these spots with Phosphorimager analysis software indicated that AFAP-110 $180-226$ showed a substantially lower amount of radioactivity incorporated, with 0.55 fold, 0.49 fold and 0.55 fold incorporation into serine, threonine and tyrosine residues, respectively. It had been previously shown that this treatment results in less than a two-fold incorporation of radioactive phosphate on all three residues, compared to treatment with the inactive phorbol ester or serum starvation alone (Qian et al., submitted). Thus, it appears that deletion of residues 180-226 blocks the hyperphosphorylation of AFAP-110 in response to PKC activation.

As in Chapter 2, affinity absorptions with a fusion protein that encodes the carboxyterminal sequences (GST-511-637) were used to determine whether AFAP-110 has undergone a conformational change. Here, GST-511-637 was used to affinity absorb cellular AFAP-110 as a 


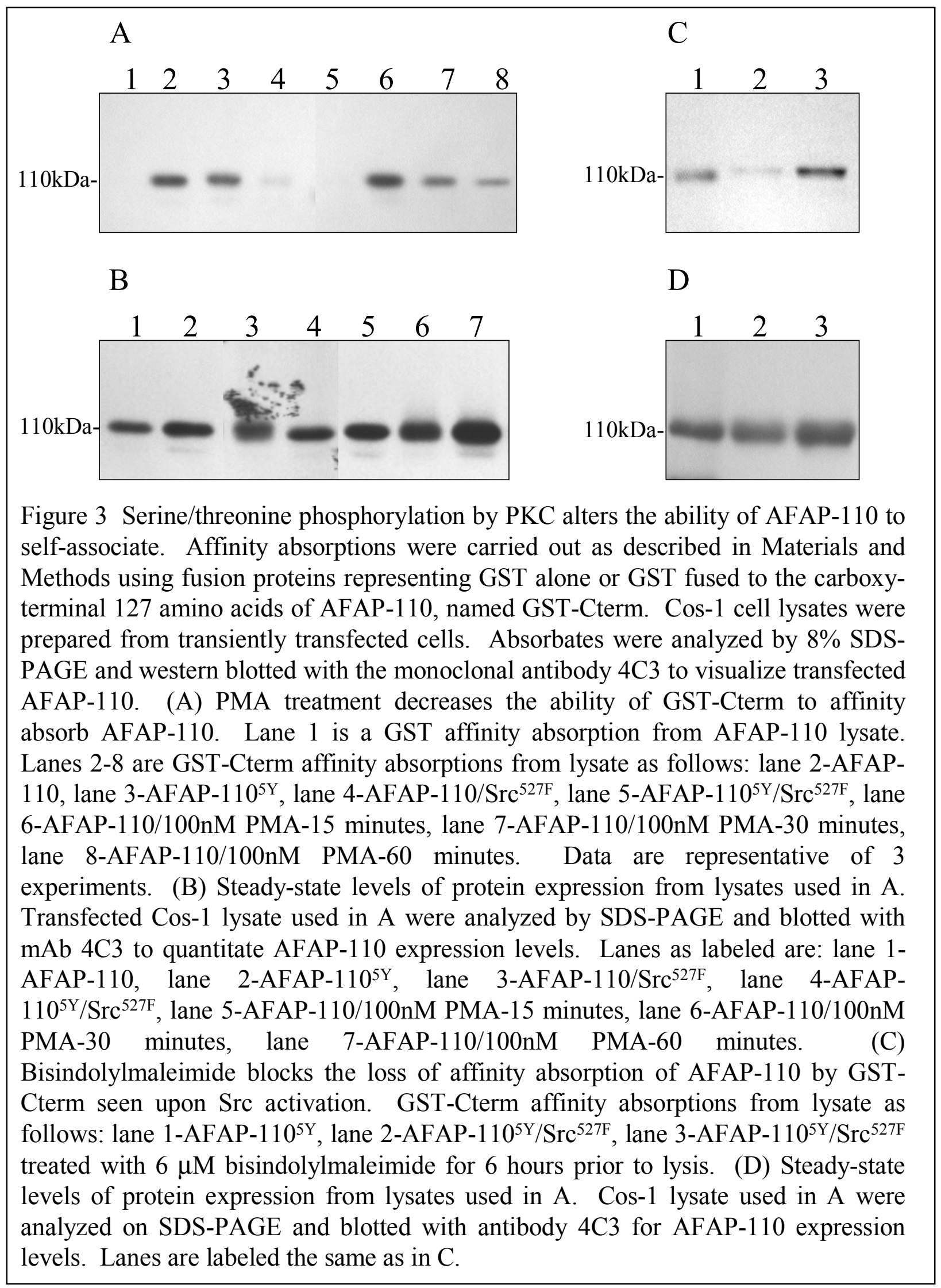


means of addressing the idea that PKC phosphorylation of AFAP-110 downstream of $\mathrm{Src}^{527 \mathrm{~F}}$ was affecting the structure of AFAP-110. Figure 3A confirms that GST-511-637 can affinity absorb cellular AFAP-110 and that co-expression of $\mathrm{Src}^{527 \mathrm{~F}}$ with AFAP-110 abrogates affinity absorption with GST-511-637. To determine whether tyrosine phosphorylation by $\mathrm{Src}^{527 \mathrm{~F}}$ was responsible for decreasing the affinity absorption of cellular AFAP-110 with GST-511-637, a mutant form of AFAP-110 was used that had all 5 tyrosines which are the sites for phosphorylation by $\mathrm{Src}^{527 \mathrm{~F}}$ mutated to phenylalanines (AFAP-110 ${ }^{5 \mathrm{Y}}$ ) (Guappone et al., 1998). GST-511-637 was able to affinity absorb $\mathrm{AFAP}^{5 \mathrm{Y}}$; however, co-expression of cellular $\mathrm{AFAP}^{5 \mathrm{Y}}$ with $\mathrm{Src}^{527 \mathrm{~F}}$ decreased the affinity absorption by GST-511-637 (Figure 2A). These data indicate that tyrosine phosphorylation of AFAP-110 is unlikely to be responsible for affecting a conformational change upon AFAP-110. Furthermore, these data suggest that $\mathrm{Src}^{527 \mathrm{~F}}$ binding is also not responsible for effecting this conformational change, as $\mathrm{Src}^{527 \mathrm{~F}}$ will not form a stable complex with $\mathrm{AFAP}^{5 Y}$ (Guappone et al., 1998).

AFAP-110 is also a strong substrate for ser/thr phosphorylation in response to $\mathrm{Src}^{527 \mathrm{~F}}$ expression (Kanner et al., 1991). In addition, AFAP-110 contains several strong consensus sequences for PKC phosphorylation (Flynn et al., 1993). To determine if PMA-activatable ser/thr kinases (which includes PKC) were responsible for altering the conformation of AFAP110, Cos-1 cells over-expressing AFAP-110 were treated with 100nM PMA. Affinity absorption with GST-511-637 demonstrated a reduction in affinity absorption in a time-dependent fashion (Figure 3A). Figure 3B shows that equivalent amounts of AFAP-110 within the lysates were used for these affinity absorptions. These data indicate that a PMA activatable ser/thr kinase may be capable of affecting the conformation of AFAP-110 and precluding affinity absorption by GST-511-637. This change in self-association is also coincident with an increase in the 
phosphorylation of AFAP-110 on serine and threonine residues in these cells (Qian submitted). It has been demonstrated that some PMA activatable PKC isoforms, such as PKC $\alpha$, are also activated by $\operatorname{Src}^{527 F}$ (Delage et al., 1993). These data indicate that perhaps a classical PKC isoform, such as $\alpha$, was responsible for effecting the conformation of AFAP-110 and decreasing affinity absorption with GST-511-637. To further test this hypothesis, cells expressing AFAP$110^{5 \mathrm{Y}}$ and $\mathrm{Src}^{527 \mathrm{~F}}$ were treated with the specific PKC inhibitor bisindolylmaleimide I ( $6 \mu \mathrm{M}$ for 6 hours) in an effort to block PKC activity. Figure 3C demonstrates that treatment with bisindolylmaleimide I will enable GST-511-637 to affinity absorb AFAP-110 from cell lysates that were treated with either PMA (100 nM for 1 hour) or were co-expressed with $\mathrm{Src}^{527 \mathrm{~F}}$. Figure 3D shows that similar amounts of lysate were used for this experiment. These data indicate that changes in conformation of AFAP-110 occur in response to $\mathrm{Src}^{527 \mathrm{~F}}$ signaling or PMA treatment and appear to be directed by PKC activation. Thus, we hypothesized that a ser/thr phosphorylation event, directed by either $\mathrm{Src}^{527 \mathrm{~F}}$ or PMA and likely from PKC, could be responsible for affecting AFAP-110 conformation.

PKC-phosphorylated AFAP-110 activates Src in vitro in an SH3-dependent fashion.

The activation of Src by an AFAP-110 mutant (AFAP-110 ${ }^{\text {Slzip }}$ ) was previously reported to require the SH3-binding motif of AFAP-110, prompting the hypothesis that Src may be activated by AFAP-110 in SH3 domain-dependent fashion. In addition, AFAP-110 $0^{\Delta l z i p}$ exhibits the conformational change seen upon both Src and PKC activation. Thus it was logical to ask if AFAP-110 could activate Src, and do so in a PKC phosphorylation-dependent manner in response to this conformational change. To test this potential, in vitro kinase assays with 
purified recombinant AFAP-110 were performed by Dr. Mingyao Liu. These results, to be included with this work in a manuscript being prepared currently, indicate that PKC phosphorylation of recombinant purified AFAP-110 induces the activation of cSrc in vitro. This experiment involves the incubation of the recombinant protein with cSrc which has been immunoprecipitated from cellular lysate, and thus is in the repressed state for catalytic activity. The ability of recombinant AFAP-110 to activate cSrc also required the integrity of the SH3 domain, as recombinant AFAP-110 $71 \mathrm{~A}$, a mutant which fails to interact with Src, failed to activate cSrc upon phosphorylation by PKC. Thus, AFAP-110 may activate Src directly upon phosphorylation by PKC, thereby prompting us to examine the potential for PKC to activate Src.

Expression of active PKC results in the activation of Src in vivo.

To determine the validity of the in vitro results, we first asked if the activation of PKC in cells results in the activation of Src. Immunofluorescence labeling of cells with phospho-specific antibodies indicates that this is the case, as seen in Figure 4. C3H10T1/2 cells were transfected with Flag-tagged myristoylated PKC $\alpha$, a constitutively active form of the enzyme previously shown to direct cytoskeletal rearrangements. This form of PKC has been shown to maintain constitutive kinase activity by virtue of its membrane association (Bazzi and Nelsestuen, 1988). Labeling these cells with anti-phosphotyrosine antibodies indicates an increased immunoreactivity with the antibody (Figure 4, panel A). Thus, cellular proteins are hyperphosphorylated on tyrosine residues in response to PKC activation. This hyperphosphorylation is seen only in cells expressing Flag-myrPKC, which also display a reorganization of the actin cytoskeleton, compared to non-transfected cells (Figure 4, panel C). 
Similar results are seen upon labeling with antibodies reactive with active Src family kinases.

Figure 4, panel D shows the results seen upon labeling similar cells with anti-SrcP(Y416), which indicate an increase in Src family kinase activity compared to non-transfected surrounding cells. Again, these cells display a reorganization of the cytoskeleton (Figure 4, panel F). These results substantiate the hypothesis that Src is activated in response to PKC activation.

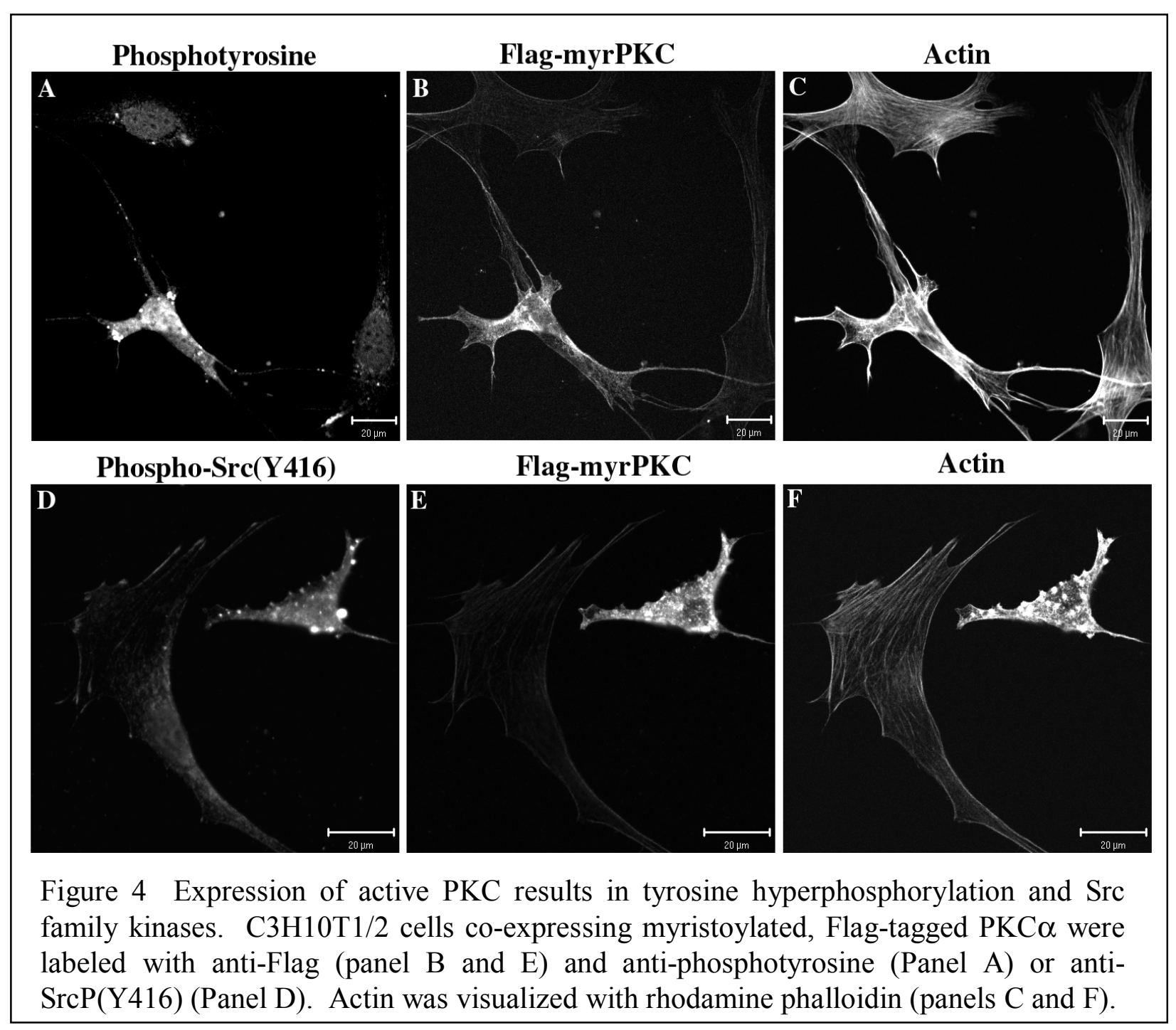


The activation of Src in response to PKC activation requires both the PH domain-mediated interactions between PKC and AFAP-110 as well as SH3 interactions between AFAP-110 and Src.

As the proposed mechanism of Src activation by PKC involves AFAP-110, GFP-tagged forms of AFAP-110 were employed to examine the potential requirement for AFAP-110 in PKC-directed Src activation as well as cytoskeletal rearrangements. The evidence presented so far indicate that both SH3 binding between AFAP-110 and Src and PH domain-directed phosphorylation of AFAP-110 by PKC may be necessary for the direct activation of Src by AFAP-110. We used AFAP-110 mutants deficient in both interactions to test this mechanism in cells. C3H10T1/2 cells were cotransfected with these forms of AFAP-110 and myristoylated, Flag-tagged PKC $\alpha$. Co-expression of GFP-AFAP-110 and Flag-myrPKC $\alpha$ resulted in the hyperphosphorylation of cellular proteins on tyrosine, as seen in Figure 6, panel A. These cells also displayed an increase in Src family kinase activation, as well (Figure 5, panel A). Cytoskeletal rearrangements were noted upon examination of GFP-AFAP-110 (Figure 5, panel C and Figure 6, panel C) in these cells, which consistently co-localizes with these rearranged actin structures. GFP-AFAP-110 ${ }^{71 \mathrm{~A}}$, shown above to block the activation of Src by AFAP-110 in vitro, co-expressed with Flag-myrPKC $\alpha$ blocks the increased tyrosine phosphorylation of cellular proteins (Figure 6, panel D) and the increased activity of Src family kinases (Figure 5, panel D) in C3H10T1/2 cells. Likewise, cytoskeletal rearrangements were blocked, as GFP$110^{71 \mathrm{~A}}$ colocalized with actin filaments (Figure 5, panel F and Figure 6, panel F). These cells also displayed levels of lamellipodia, filopodia and ruffles comparable to non-transfected cells. Therefore, SH3 interactions between AFAP-110 and Src are required for the activation of Src in 


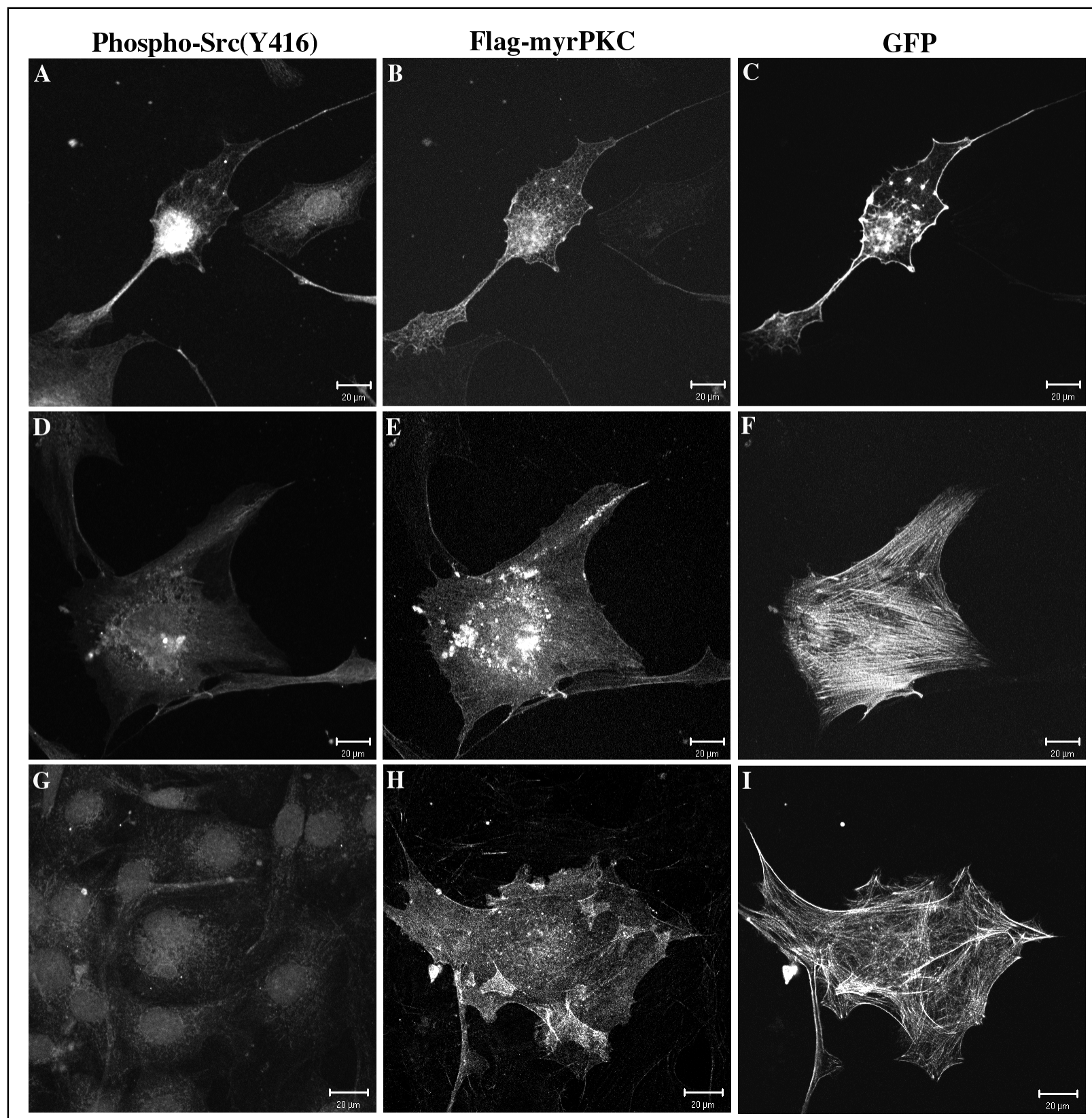

Figure 5. The activation of Src family kinases by $\mathrm{PKC}$ requires the integrity of both the SH3 binding motif and PH domain of AFAP-110. C3H10T1/2 cells co-expressing GFPAFAP-110 (Panel C) and myristoylated, Flag-tagged PKC $\alpha$ were labeled with anti-Flag (panel B ) and anti-phospho-Src(Y416) (Panel A). Cells co-expressing GFP-AFAP$110^{71 \mathrm{~A}}$ (Panel F) and myristoylated, Flag-tagged $\mathrm{PKC} \alpha$ were similarly labeled to visualize PKC (Panels E) and anti-phospho-Src(Y416) (Panel D). Additionally, cells expressing GFP-AFAP-110 $0^{\Delta 180-226}$ (Panels I) with Flag-myrPKC $\alpha$ (Panels H). These cells were also labeled with anti-phosphotyrosine (Panel G). Representative images are shown. 
response to PKC. GFP-AFAP-110 $0^{\Delta 180-226}$ co-expressed with Flag-myrPKC $\alpha$ also blocks both the increased tyrosine phosphorylation of cellular proteins (Figure 6, panel G) and the increased activity of Src family kinases (Figure 5, panel G). Additionally, the expression of this mutant blocks cytoskeletal rearrangements normally seen upon expression of active PKC. Figure 5, panel I and Figure 6, panel I indicate the colocalization of GFP-AFAP- $110^{\Delta 180-226}$ with copious actin filaments. Normal levels of lamellipodia, filopodia and membrane ruffles are noted in these cells, as well. Thus, the PH domain-dependent phosphorylation of AFAP-110 and subsequent conformational change may be necessary for both the activation of Src as well as cytoskeletal rearrangements by PKC. 


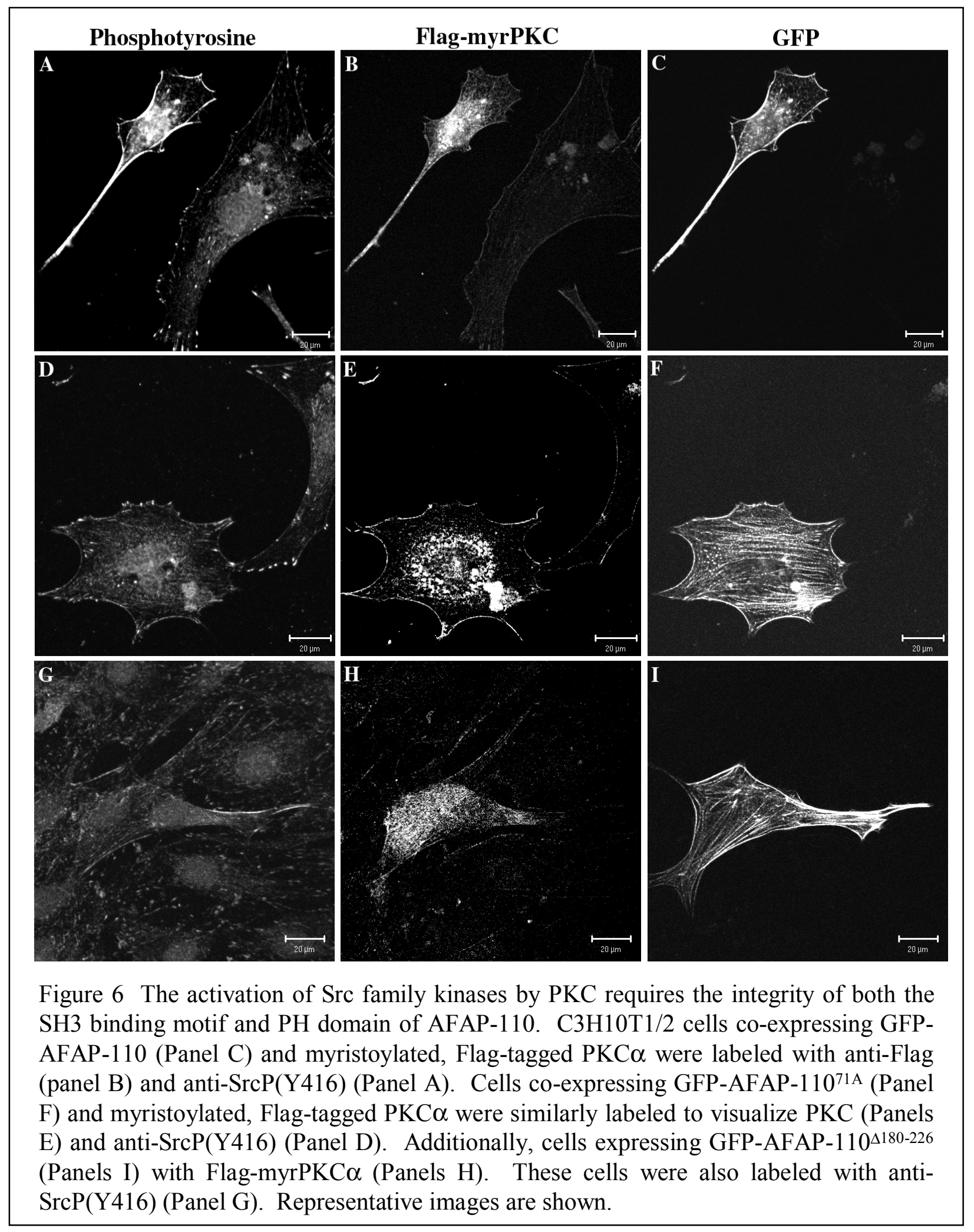




\section{Discussion}

AFAP-110 appears to be a PKC binding partner, through interactions involving the amino-terminal PH domain of AFAP-110. Careful examination of this PH domain has revealed homology to other $\mathrm{PH}$ domains which have been shown to interact with PKC. Although $\mathrm{PH}$ domains generally share low levels of sequence homology, they share a well conserved tertiary structure which can be useful for alignment of the key structural elements. This is helpful, as these domains are generally 100 amino acids in length and contain 7 beta strands and 1 or 2 alpha helices with intervening sequences of variable lengths (Shaw, 1996). As shown in the introduction, a model of the amino-terminal PH domain based on $\beta$-Spectrin (Macias et al., 1994), with which it shares highest homology, has allowed for the comparison of this domain with PKC-binding PH domains. Based on several PH domains which bind PKC, including those from Btk, Dynamin, $\beta$-Spectrin as well as others, a consensus has been generated (Waldron et al., 1999; Yao et al., 1997). The amino-terminal PH domain fits this consensus well, with similar residues in 7 of the 8 consensus positions. The carboxy-terminal PH domain, which is shown in Figure 1 to be deficient for interactions with PKC, shares only 4 of these consensus positions. Similar to the experiments reported involving $\mathrm{PKC}$ and the $\mathrm{PH}$ domain from Btk, deletion of a specific portion of the amino-terminal PH domain results in the decreased ability of the fusion protein to affinity absorb PKC. This mutant fusion protein retains a portion of the $\beta 1$ and $\beta 2$ sheets of the $\mathrm{PH}$ domain, structures which contain the consensus thought to be responsible for PKC binding in the case of the Btk PH domain (Yao et al., 1997). Thus, the interaction between the amino-terminal PH domain of AFAP-110 and PKC may require sequences in addition to the consensus. Alternatively, the deletion of residues 180-226, carboxy- 
terminal to the consensus, may disrupt the tertiary structure of the $\mathrm{PH}$ domain so that it is incapable of binding PKC although it retains the sequence necessary for this activity.

The amino-terminal PH domain is capable of affinity absorbing the members of the classical family of PKCs, PKC $\alpha, \beta$ and $\gamma$, as well as the atypical PKC $\lambda /$. To the authors' knowledge, the interaction between $\mathrm{PKC} \lambda / \mathrm{l}$ and $\mathrm{AFAP}-110$ represents a novel result that diverges from previous reports, which have not demonstrated an interaction between PH domains and atypical PKC's $\lambda /$. These reports have shown that PH domains do show preference for closely related PKC isoforms, even among the PKC families. For instance, the PH domain of PKD shows a much

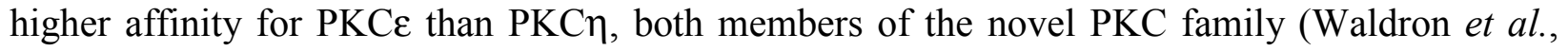

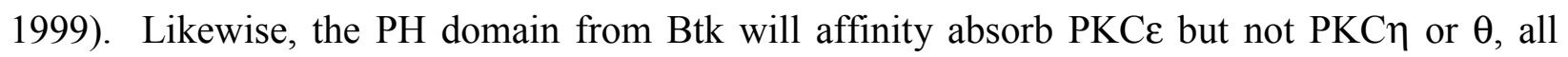
related members of the novel PKC family (Yao et al., 1994). Thus, the amino-terminal PH domain from AFAP-110 is consistent with other PH domains in its ability to discriminate between PKC family members. The significance of this discrimination may be revealed by examining the function of PKC isoforms. The PKC isoforms shown here to interact with AFAP110 are thought to be involved in cytoskeletal rearrangements. PKC $\alpha$ has been well established as an important factor in cytoskeletal rearrangements in various cell types, including lung epithelial cells (Dwyer-Nield et al., 1996), fibroblasts (Lin et al., 1996), glioma cells (Douglas et al., 1997), smooth muscle cells (Haller et al., 1995; Wang et al., 1997), endometrial adenocarcinoma cells (Carter et al., 1998), and breast cells (Sun and Rotenberg, 1999). The other members of the classical PKC family (PKC $\beta$ and $\gamma$ ) have not been studied in the same detail; however most of the aforementioned studies which inhibit PKC $\alpha$ activity employ inhibitors which block the entire classical PKC family. PKC $\lambda / \imath$ has not been specifically shown to affect cytoskeletal rearrangements; however inhibition of the entire atypical PKC family $(\lambda / \imath$ 
and $\zeta$ ) can block Ras-mediated cytoskeletal change in fibroblasts (Uberall et al., 1999). Thus the PKC isoforms which appear to be capable of interacting with AFAP-110 are also involved in cytoskeletal rearrangements.

The mechanism whereby PKC directs cytoskeletal rearrangements is not well understood. Most previous studies have investigated actin-binding proteins which are PKC substrates for direct effects on actin structures. Here, we uniquely show that PKC phosphorylation of the actin-binding protein AFAP-110 results in the activation of Src, providing an indirect means of facilitating cytoskeletal rearrangements. This activation of Src requires SH3 interactions between AFAP-110 and Src. This method of activation of Src is not without precedent. Two viral proteins, the Nef protein produced by HIV, and the Tip protein from Herpesvirus saimiri strain $484 \mathrm{C}$, activate Src directly by engaging the SH3 domain and relieving the intramolecular constraints which repress kinase activity (Briggs et al., 1997; Moarefi et al., 1997); (Lund et al., 1997). Young, et al. have recently reported that the SH2 and SH3 intramolecular interactions which repress Src activity act as a "snap lock" in an all-or-nothing type of inhibition, supporting the idea that SH3 engagement alone would suffice to activate Src (Young et al., 2001). Thus it is feasible that AFAP-110 would activate Src in a similar manner.

Other reports have indicated the potential for Src to be activated upon the activation of PKC. Phorbol ester treatment of Swiss 3T3 fibroblasts reportedly activated Src three fold, a result which was blocked by PKC inhibition with bisindolylmaleimide I (Rodriguez-Fernandez and Rozengurt, 1996). Likewise, the activation of Src upon treatment of Swill 3 T3 cells with bombesin or PDGF was partially dependent upon PKC activity (Salazar and Rozengurt, 1999). In another cell system, cultured mast cells showed increased Src activity upon over-expression of 
PKC $\delta$ (Song et al., 1998). These reports provide some evidence for the activation of Src by PKC.

The specific results presented here indicate AFAP-110 is a PKC binding partner and that the PH-domain directed phosphorylation of AFAP-110 by PKC results in the direct activation of Src by SH3 interactions between AFAP-110 and Src. These results outline a unique mechanism for the activation of a potent oncogene by pathways heretofore thought to function downstream of this oncogene. The activation of Src by RTK's in response to extracellular stimuli has been accepted to play a role in the etiology of several types of cancer. This alternate mechanism would provide for the activation of Src by tumorigenic stimuli which proceed via activation of PKC, which has been well characterized for its tumor promoting potential. As AFAP-110 is expressed in many cell types including fibroblast, epithelial and endothelial cells as well as cells of hematopoietic lineage (Flynn et al., 1995), and exists on both motility-associated structures and the cell membrane in both quiescent and transformed cells (Qian et al., 1998), it is situated to transmit signals which would result in the activation of Src by the mechanism proposed here. 


\section{Materials and Methods}

\section{Reagents and proteins}

Dulbecco's modified Eagle's medium (DMEM) and Basal Medium Eagle (BME) were obtained from Sigma. Recombinant PKC $\alpha$ was purchased from Calbiochem. Phorbol 12-myristate 13-

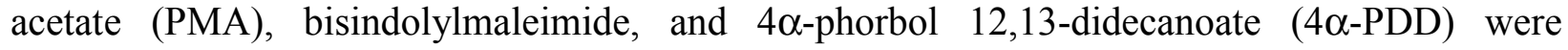
obtained from Sigma. PKC isoform antibodies were obtained from the Transduction Laboratories' PKC sampler kit, with the exception of the PKC $\zeta$ antibody, which was purchased from Calbiochem. The polyclonal pan-PKC antibody used was from Calbiochem (Ab-1). AFAP-110 antibodies 4C3 and F1 were generated and characterized as previously described (Flynn et al., 1993; Kanner et al., 1989). Phosphatidylserine, rhodamine-phalloidin and diolein were purchased from Sigma. Recombinant AFAP-110 and rAFAP-110 ${ }^{71 \mathrm{~A}}$ were purified after production as a GST bacterial fusion protein, using the PreScission Protease system (Amersham Pharmacia) as previously described (Qian et al., 2000).

\section{Phosphamino acid analysis}

Cos-1 cells were transiently transfected and grown for 60 hours. Prior to lysis, cells were serum starved overnight and incubated with $10 \mathrm{mCi} 32 \mathrm{P}$ orthophosphate, then stimulated with $100 \mathrm{nM}$ PMA for 15 minutes. Cells were washed twice with PBS and lysed with $1 \mathrm{ml}$ RIPA buffer. 1.5 $\mu 1$ monoclonal antibody 4C3 was used to immunoprecipitate AFAP-110 from the lysate, which was isolated via SDS-PAGE. The radioactive band was excised from the gel and subjected to 
partial acid hydrolysis and phosphamino acid analysis (Boyle et al., 1991). After running the isolated amino acids on 2D TLC, the plates were imaged using a Phosphorimager (Molecular Dynamics). Spots were identified by running labeled phosphoserine, phosphothreonine, and phosphotyrosine markers. Relative intensity compared to background of radiation from spots was quantitated with ImageQuant software.

Affinity absorption assays

Fusion proteins were generated, quantified and employed in absorptions as described previously (Qian et al., 1998). For western blot analysis, the absorbates were analyzed by SDS-PAGE. For experiments involving serine/threonine kinase assays, the absorbates were washed 5 times with MTPBS (4.38 g NaCl, $1.14 \mathrm{~g} \mathrm{Na}_{2} \mathrm{HPO}_{4}, 0.24 \mathrm{NaH}_{2} \mathrm{Po}_{4}$ in $500 \mathrm{ml} \mathrm{H} \mathrm{H}_{2} \mathrm{O}$, pH 7.3) + 1\% Triton, then four times with TBS. The absorbate/bead slurry was subjected to a colorimetric PKA assay (Pierce) as per protocol. For Fig. 1A-C, $20 \mu \mathrm{g}$ fusion protein and $250 \mu \mathrm{g}$ of day 18 chick embryo brain lysate was used. For Fig. 1D, $20 \mu \mathrm{g}$ fusion protein and $500 \mathrm{ng}$ recombinant PKC $\alpha$ were used.

Co-immunoprecipitation assays

Anti-Flag antibody was used to immunoprecipitate Flag-tagged wild-type PKC $\alpha$ from Cos-7 cell lysate. Immunoprecipitate was subjected to SDS-PAGE and transferred proteins were labeled on PVDF with anti-GFP antibody. 


\section{Cell culture}

Cos-1 cells were maintained and transfected as previously described (Guappone and Flynn, 1997). C3H10T1/2 cells were cultured as previously described (Qian et al., 2000).

\section{Immunofluorescence}

Transient transfections of $\mathrm{C} 3 \mathrm{H} 10 \mathrm{~T} 1 / 2$ cells were carried out using Effectene (Qiagen) as per. Cells were fixed and permeabalized as previously described (Qian et al., 1998). For cells transfected with Flag-tagged PKC $\alpha$, rhodamine phalloidin (1:1000) and anti-Flag ab were used. Anti-mouse Alexa 488 was used to visualize the anti-Flag ab. Anti-ptyr and antiSrc(pY416) were used in separate experiments to label tyrosine-phosphorylated proteins and active Src family kinases, respectively. These polyclonal antibodies were visualized with anti-rabbit Alexa 633. For cells co-transfected with Flag-tagged PKC $\alpha$ and GFP-tagged forms of AFAP-110, antiFlag, anti-ptyr and antiSrc(pY416) were used as above. In this instance, anti-mouse TRITC was used to visualize anti-Flag antibody, while phospho-specific antibodies were labeled as above. Cells were washed and mounted on slides with Fluoromount (Fisher). A Zeiss LSM 510 microscope was used to gather images. Scale bars were generated and inserted by LSM 510 software. For all figures, representative cells are shown ( $>100$ cells examined per image shown). 
References

Baraldi,E, Carugo,KD, Hyvonen,M, Surdo,PL, Riley,AM, Potter,BV, O'Brien,R, Ladbury,JE, and Saraste,M. (1999). Structure. Fold. Des, 7, 449-460.

Baserga,R, Sell,C, Porcu,P, and Rubini,M. (1994). Cell Prolif., 27, 63-71.

Bazzi,MD and Nelsestuen,GL. (1988). Biochem. Biophys. Res. Commun., 152, 336-343.

Bolen,JB, Veillette,A, Schwartz,AM, DeSeau,V, and Rosen,N. (1987). Proc. Natl. Acad. Sci. U. S. $A, \mathbf{8 4}, 2251-2255$.

Boyle,WJ, van der,GP, and Hunter,T. (1991). Methods Enzymol., 201, 110-149.

Briggs,SD, Sharkey,M, Stevenson,M, and Smithgall,TE. (1997). J. Biol. Chem., 272, 1789917902.

Carter,CA, Parham,GP, and Chambers,T. (1998). Pathobiology, 66, 284-292.

Cartwright,CA, Kamps,MP, Meisler,AI, Pipas,JM, and Eckhart,W. (1989). J. Clin. Invest, 83, 2025-2033.

Cartwright,CA, Meisler,AI, and Eckhart,W. (1990). Proc. Natl. Acad. Sci. U. S. A, 87, 558-562.

Delage,S, Chastre,E, Empereur,S, Wicek,D, Veissiere,D, Capeau,J, Gespach,C, and Cherqui,G. (1993). Cancer Res., 53, 2762-2770.

Deman,J and Van Larebeke,N. (2001). Tumour. Biol., 22, 191-202.

Douglas,DN, Fink,HS, Rose,SD, Ridgway,ND, Cook,HW, and Byers,DM. (1997). Biochim. Biophys. Acta, 1356, 121-130.

Dwyer-Nield,LD, Miller,AC, Neighbors,BW, Dinsdale,D, and Malkinson,AM. (1996). Am. J. Physiol, 270, L526-L534.

Flynn,DC, Koay,TC, Humphries,CG, and Guappone,AC. (1995). J. Biol. Chem., 270, 38943899.

Flynn,DC, Leu,TH, Reynolds,AB, and Parsons,JT. (1993). Mol. Cell Biol., 13, 7892-7900.

Guappone,AC and Flynn,DC. (1997). Mol. Cell Biochem., 175, 243-252.

Guappone,AC, Weimer,T, and Flynn,DC. (1998). Mol. Carcinog., 22, 110-119.

Haller,H, Lindschau,C, Quass,P, Distler,A, and Luft,FC. (1995). Circ. Res., 76, 21-29.

Kanner,SB, Reynolds,AB, and Parsons,JT. (1989). J. Immunol. Methods, 120, 115-124. 
Kanner,SB, Reynolds,AB, Wang,HC, Vines,RR, and Parsons,JT. (1991). EMBO J., 10, 16891698.

Kim,H and Muller,WJ. (1999). Exp. Cell Res., 253, 78-87.

Lazar-Molnar,E, Hegyesi,H, Toth,S, and Falus,A. (2000). Cytokine, 12, 547-554.

Lin,X, Tombler,E, Nelson,PJ, Ross,M, and Gelman,IH. (1996). J. Biol. Chem., 271, 2843028438.

Loeb,LA. (2001). Cancer Res., 61, 3230-3239.

Lund,T, Medveczky,MM, and Medveczky,PG. (1997). J. Virol., 71, 378-382.

Macias,MJ, Musacchio,A, Ponstingl,H, Nilges,M, Saraste,M, and Oschkinat,H. (1994). Nature, 369, 675-677.

Moarefi,I, LaFevre-Bernt,M, Sicheri,F, Huse,M, Lee,CH, Kuriyan,J, and Miller,WT. (1997). Nature, 385, 650-653.

Qian,Y, Baisden,JM, Westin,EH, Guappone,AC, Koay,TC, and Flynn,DC. (1998). Oncogene, 16, 2185-2195.

Qian,Y, Baisden,JM, Zot,HG, Van Winkle,WB, and Flynn,DC. (2000). Exp. Cell Res., 255, 102113.

Rodriguez-Fernandez,JL and Rozengurt,E. (1996). J. Biol. Chem., 271, 27895-27901.

Rodriguez,MM, Ron,D, Touhara,K, Chen,CH, and Mochly-Rosen,D. (1999). Biochemistry, 38, 13787-13794.

Rosen,N, Bolen,JB, Schwartz,AM, Cohen,P, DeSeau,V, and Israel,MA. (1986). J. Biol. Chem., 261, 13754-13759.

Salazar,EP and Rozengurt,E. (1999). J. Biol. Chem., 274, 28371-28378.

Shaw,G. (1996). Bioessays, 18, 35-46.

Song,JS, Swann,PG, Szallasi,Z, Blank,U, Blumberg,PM, and Rivera,J. (1998). Oncogene, 16, 3357-3368.

Sun,XG and Rotenberg,SA. (1999). Cell Growth Differ., 10, 343-352.

Uberall,F, Hellbert,K, Kampfer,S, Maly,K, Villunger,A, Spitaler,M, Mwanjewe,J, BaierBitterlich,G, Baier,G, and Grunicke,HH. (1999). J. Cell Biol., 144, 413-425.

Waldron,RT, Iglesias,T, and Rozengurt,E. (1999). J. Biol. Chem., 274, 9224-9230.

Wang,S, Desai,D, Wright,G, Niles,RM, and Wright,GL. (1997). Exp. Cell Res., 236, 117-126. 
Yao,L, Kawakami,Y, and Kawakami,T. (1994). Proc. Natl. Acad. Sci. U. S. A, 91, 9175-9179.

Yao,L, Suzuki,H, Ozawa,K, Deng,J, Lehel,C, Fukamachi,H, Anderson,WB, Kawakami,Y, and Kawakami,T. (1997). J. Biol. Chem., 272, 13033-13039.

Young,MA, Gonfloni,S, Superti-Furga,G, Roux,B, and Kuriyan,J. (2001). Cell, 105, 115-126. 
Discussion 
The work presented here addresses one role of AFAP-110 in cytoskeletal rearrangements. Overall, the results indicate that AFAP-110 can activate cSrc in response to cellular signals, thereby inducing cytoskeletal rearrangements in an indirect manner. This is summarized in Figure 1.

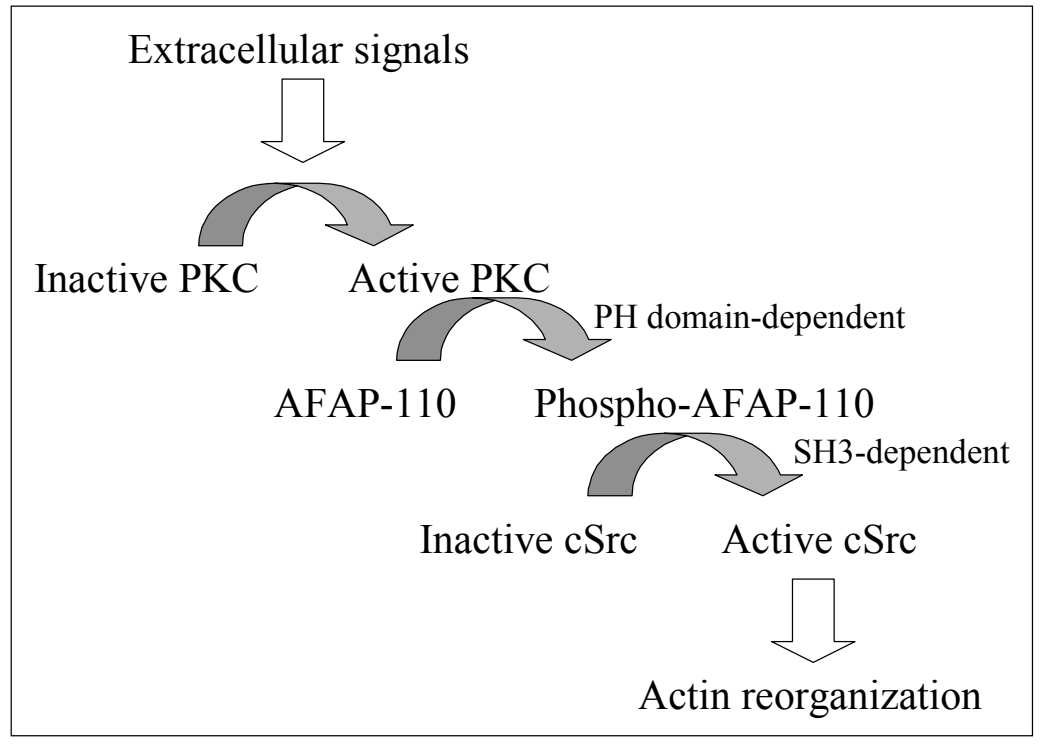

Figure 1 Proposed pathway by which AFAP-110 is involved in cytoskeletal rearrangements in an indirect manner.

The mechanism employed by AFAP-110 to activate cSrc appears to proceed through one intrinsic quality of AFAP-110, in this case the ability of AFAP-110 to engage the SH3 domain of cSrc. This interaction results in the activation of cSrc, presumably by interfering with and relieving the repressive intramolecular interactions involving the SH3 domain of cSrc. In support of this potential, deletion or mutation of the SH3 domain from cSrc results in activation (Gonfloni et al., 1997; Reynolds et al., 1992; Weil and Veillette, 1994; Wright et al., 1994). As discussed earlier, the potential for the activation of Src family kinases by SH3 engagement is not without precedent. The HIV Nef protein and Herpesvirus Tip protein have been shown to activate Src family members in this fashion (Collette et al., 2000; Hartley et al., 1999; Moarefi et 
al., 1997). Both of these proteins transform cells, and the Tip protein is a requirement for the induction of tumors by the Herpesvirus (Duboise et al., 1998). As many viral proteins are thought to adapt cellular mechanisms to suit their own needs, its seems likely that a cellular Src activator of this class would exist. Furthermore, logic would dictate that this activator would function in this capacity only upon the receipt of specific signals, as Src is normally maintained in a tightly repressed state. AFAP-110 fits both of these criteria, as it appears to activate cSrc via SH3 interactions and this ability is revealed upon phosphorylation by PKC. This phosphorylation correlates with a conformational change in AFAP-110, as measured by a change in self-association. Thus, AFAP-110 is altered in response to this specific signal, which could permit AFAP-110 to activate cSrc via SH3 interactions.

The potential for PKC activation to induce the activation of $\mathrm{cSrc}$ is supported by previous reports. The activation of PKC by bombesin or phorbol ester resulted in the increased activity of Src in two separate reports (Rodriguez-Fernandez and Rozengurt, 1996; Salazar and Rozengurt, 1999). This mechanism may be utilized by cellular signals to activate both PKC and cSrc. The end result is the activation of both kinases and the reorganization of cytoskeletal structures. These kinases appear to signal in both directions, as cSrc activation results in PKC activation (Qureshi et al., 1991; Zang et al., 1995). As AFAP-110 is required for the activation of Src by $\mathrm{PKC}$, it is positioned to provide a crucial, bi-directional signaling link between these two kinases. This idea is supported by careful consideration of the adapter nature of AFAP-110 and the proteins it may bind to bring together into complex.

The potential for AFAP-110 to fill the role of a cSrc activator is substantiated by consideration of the subcellular localization of Src, PKC and AFAP-110. PKC is recruited to the cell membrane upon its activation, where cSrc is anchored by myristoylation. AFAP-110 also 
appears enriched at the cell membrane upon PKC activation, indicating it is positioned to efficiently activate Src in response to phosphorylation by PKC. One result of the activation of both of these proteins is the formation of actin-rich structures at the cell membrane, namely lamellipodia, membrane ruffles and filopodia. In both Src and PKC-transformed cells, AFAP110 is consistently enriched in these structures. As AFAP-110 is also hyperphosphorylated in these cells by PKC, it seems likely that AFAP-110 would be hyperphosphorylated at the sites where it is enriched and where PKC is activated, namely the cell membrane.

The ability of AFAP-110 to bind both Src and PKC directly suggests the possibility of the formation of a large signaling complex. As all three proteins are enriched at the cell membrane upon the activation of either Src or PKC, the likelihood that this complex would form is increased in this event. The presence of additional binding motifs in AFAP-110 indicates other proteins may enjoin this complex as well. One such protein is the receptor for activated C kinase, RACK1. This protein was identified as a PKC binding partner which bound to and stabilized active PKC at the cell membrane (Ron et al., 1994). RACK1 is maintained at the cell membrane by a myristoylation signal, much like Src. Work along two veins indicates that RACK1 would likely participate in this signaling complex involving AFAP-110, Src and PKC. Firstly, RACK1 has been shown to bind PH domains, including those from Dynamin and $\beta$ Spectrin, which share homology with the amino-terminal PH domain from AFAP-110 (Rodriguez et al., 1999). RACK1 appears to bind PH domain through one or more of its WD40 repeats, protein binding modules characterized by a tryptophan and adjacent aspartate every 40 residues. Work with other PH domains that bind WD40 repeat-containing proteins has allowed for the generation of a consensus binding sequence, which covers a large portion of the carboxyterminal region of the $\mathrm{PH}$ domain. This consensus is included in Appendix V, which also 
compares the PH domain of AFAP-110. Both PH domains fit this consensus well, indicating the potential for binding WD40 repeat-containing proteins, including RACK1. The second area of work reported in the literature impacting the potential formation of AFAP-110-directed signaling complexes involves RACK1 and Src. Although RACK1 was identified as a binding partner for PKC, recent work has demonstrated that RACK1 also binds Src directly (Chang et al., 1998). This interaction inhibits Src kinase activity, and over-expression of RACK1 in cells decreases their rate of growth, thought to be due to Src inhibition. More recently, it was found that PKC activation enhances the interaction between Src and RACK1 (Chang et al., 2001). As activated PKC is bound to RACK1, this strongly supports the idea that a signaling complex consisting of Src and PKC could form. To investigate the potential for AFAP-110 to participate in this complex, affinity absorptions with fusion proteins representing the amino-terminal $\mathrm{PH}$ domain were used to affinity absorb RACK1 from chick brain lysate. The result, shown in Appendix V, indicates RACK1 is affinity absorbed by this fusion protein. It is unlikely that this result is due to the absorption of PKC, with the presumption that RACK1 associated with PKC would be a false positive result for direct interactions. This is because all reports of the PKC/RACK1 interaction have indicated an inability to co-immunoprecipitate RACK1 with anti-PKC antibodies (Ron et al., 1999). It is possible, however, that other proteins besides PKC could bridge the PH domain of AFAP-110 and RACK1 in this assay. Anti-RACK1 antibodies revealed that AFAP-110 can be co-immunoprecipitated from cell lysate, as well. This is included in appendix V. It seems likely, therefore, that large signaling complexes may form consisting of at least RACK1, Src, PKC and AFAP-110. There may be other proteins involved in these complexes, as well. The presence of these proteins at the cell membrane indicates this 
type of complex could play necessary roles in multiple cellular functions, including the reorganization of the actin cytoskeleton as addressed here.

Evidence exists for the role of AFAP-110 in the formation of actin-rich cell membrane structures in cell types other than cultured fibroblasts, as well. AFAP-110 is found in lysate from multiple tissue types, including breast, colon, and epithelial tissues. Epithelial cells grown in culture reach a state of confluency and arrest their growth, to maintain a continuous sheet, analogous to epithelial sheets found in organisms. At the cell-cell junctions, multiple proteins are involved in maintaining the integrity of the sheet and signaling for the cell to arrest its growth upon contacting another cell. Actin is found here and is thought to be involved in connecting the cytoskeletal structures between cells to provide an overall strength to the epithelial sheet. Interestingly, AFAP-110 is found on the actin filaments in these cells, but is absent from the actin-rich cell-cell borders (Appendix VI). Additionally, during subconfluent stages or upon the activation of PKC with PMA in confluent sheets, AFAP-110 is found in actin-rich structures at the cell membrane. These structures represent lamellipodia, membrane ruffles and filopodia. Published results have indicated PKC plays a role in the migration of epithelial cells in several capacities, including the induction of motility structures and the internalization of structures which anchor the cells to one another, preventing cell migration $(\mathrm{Ng}$ et al., 1999; Sun and Rotenberg, 1999). As AFAP-110 appears at cell-cell borders upon the activation of PKC, it seems plausible that AFAP-110 may play a role in the alteration of actin-rich membrane structures in epithelial cells.

AFAP-110 can bind actin filaments directly and can multimerize. Therefore it represents a potential actin bundling protein. Changes in the self-association of AFAP-110 in response to PKC phosphorylation, included in Appendix IV, indicate that AFAP-110's ability to bundle actin 
filaments could be regulated by this signal. This appears to be the case, as shown in a manuscript submitted by Dr. Yong Qian indicating an increase in direct bundling of actin filaments by microscopic analysis. In this work, PKC phosphorylation of AFAP-110 resulted in the dramatic increase of the bundling of actin filaments polymerized in vitro. Experiments aimed at dissecting the self-association of AFAP-110 and the mechanism of the regulation of this property are described in Appendix IV. These data indicate that AFAP-110 self-association is directed by complex interactions involving at least two separate sites of interaction. Further work is necessary to determine if this property of AFAP-110 is responsible for the activation of Src in addition to its role in direct cytoskeletal effects.

In summary, this dissertation describes AFAP-110 as a potential activator for cSrc in response to cellular signals, namely PKC phosphorylation. This result is unique, as this represents the first example of a cellular protein shown to function in such a fashion. The existence of this type of a signaling pathway as this would allow for the activation of cSrc in response to signals not previously known to induce this result. As the PH domain of AFAP-110 is required for this function, this domain provides a potential target for the abrogation of interactions necessary for the potentiation of this signal. This could prove useful in the inhibition of cell migration, particularly in the treatment of metastatic disease. 


\section{References}

Chang,BY, Chiang,M, and Cartwright,CA. (2001). J. Biol. Chem., 276, 20346-20356.

Chang,BY, Conroy,KB, Machleder,EM, and Cartwright,CA. (1998). Mol. Cell Biol., 18, 32453256.

Collette,Y, Arold,S, Picard,C, Janvier,K, Benichou,S, Benarous,R, Olive,D, and Dumas,C. (2000). J. Biol. Chem., 275, 4171-4176.

Duboise,SM, Lee,H, Guo,J, Choi,JK, Czajak,S, Simon,M, Desrosiers,RC, and Jung,JU. (1998). J. Virol., 72, 2607-2614.

Gonfloni,S, Williams,JC, Hattula,K, Weijland,A, Wierenga,RK, and Superti-Furga,G. (1997). EMBO J., 16, 7261-7271.

Hartley,DA, Hurley,TR, Hardwick,JS, Lund,TC, Medveczky,PG, and Sefton,BM. (1999). J. Biol. Chem., 274, 20056-20059.

Moarefi,I, LaFevre-Bernt,M, Sicheri,F, Huse,M, Lee,CH, Kuriyan,J, and Miller,WT. (1997). Nature, 385, 650-653.

Ng, T, Shima,D, Squire,A, Bastiaens,PI, Gschmeissner,S, Humphries,MJ, and Parker,PJ. (1999). EMBO J., 18, 3909-3923.

Qureshi,SA, Joseph,CK, Rim,M, Maroney,A, and Foster,DA. (1991). Oncogene, 6, 995-999.

Reynolds,PJ, Hurley,TR, and Sefton,BM. (1992). Oncogene, 7, 1949-1955.

Rodriguez-Fernandez,JL and Rozengurt,E. (1996). J. Biol. Chem., 271, 27895-27901.

Rodriguez,MM, Ron,D, Touhara,K, Chen,CH, and Mochly-Rosen,D. (1999). Biochemistry, 38, 13787-13794.

Ron,D, Chen,CH, Caldwell,J, Jamieson,L, Orr,E, and Mochly-Rosen,D. (1994). Proc. Natl. Acad. Sci. U. S. A, 91, 839-843.

Ron,D, Jiang,Z, Yao,L, Vagts,A, Diamond,I, and Gordon,A. (1999). J. Biol. Chem., 274, 2703927046.

Salazar,EP and Rozengurt,E. (1999). J. Biol. Chem., 274, 28371-28378.

Sun,XG and Rotenberg,SA. (1999). Cell Growth Differ., 10, 343-352.

Weil,R and Veillette,A. (1994). J. Biol. Chem., 269, 22830-22838.

Wright,DD, Sefton,BM, and Kamps,MP. (1994). Mol. Cell Biol., 14, 2429-2437.

Zang,Q, Frankel,P, and Foster,DA. (1995). Cell Growth Differ., 6, 1367-1373. 


\section{Appendix I}

Microscopic analysis using the Zeiss LSM510 
As much of my work has necessitated the use of microscopy, I have become accustomed to using this machine, located in the Anatomy department. This machine provides excellent resolution for image analysis down to a scale of around $20 \mu \mathrm{M}$. The discussion that follows relates much of the information and ideas I have gathered which have helped me in performing microscopic analysis.

There are several factors which influence the quality of images gathered upon microscopy. These include: (1) the quality of labeling, (2) the choice of secondary antibodies, and (3) the ability to gather quality representative images from the sample. As all of these issues are important and deficiency in any area can significantly decrease data quality, I will address each of these issues separately.

The quality of labeling is governed largely by the ability to create a large signal to noise ratio. The signal is the specific labeling of the protein of interest, while the noise is the nonspecific background labeling of unwanted proteins. Some background can come from autofluorescence of proteins, but this is generally extremely difficult to detect and therefore does not add to detectable noise. The most efficient way to generate signal without the possibility of generating noise is to use a fluorescent tag of your protein of interest, such as GFP or DsRed. These are proteins identified from jellyfish and sea anemones, respectively which have been optimized genetically by companies (ClonTech) for fluorescence. The use of any antibody, however, generates background noise, but this can be minimized. One way to minimize this noise is to optimize antibody concentrations using a series of dilutions to determine which antibody concentration is optimal. Another way is to block background staining with other, "sticky" blocking agents such as milk or BSA. I commonly use 5\% BSA as a vehicle for my 
antibody staining. Additionally, maintaining optimal protein expression levels in the case of transient transfections is important to maintaining a high signal.

Most labeling involves the use of antibodies, and the concentration of antibody used is the single most important factor for obtaining an adequate signal to noise ratio. This has to be determined for each antibody used individually, and is best done in control experiments before setting up investigative experiments. Much time and frustration can be avoided by this following this path. The cell type being used also influences the antibody concentration needed, as well as the type of protein being examined (endogenous vs. transient expression). Included in Table 1 are the antibody concentrations I have optimized and used for each cell type and protein type I have examined by fluorescence microscopy. These concentrations should be viewed as a guidebook for ranges of concentrations to use, as antibody affinity can change with storage or between different lots.

When labeling more than one protein, as is commonly done, the secondary antibodies chosen can greatly influence the results obtained. As the Zeiss microscope uses lasers to specifically excite certain wavelengths and optical filters to specifically gather certain wavelengths, it limits its detection of fluorescence to signals only within those wavelengths. This allows us to efficiently separate the signals generated in most cases. However, when one signal from a labeled protein extends into the detection range of a signal from another labeled protein, false data can be returned. This commonly happens with this microscope, but can be avoided. The reason this happens is because of the spectral properties of the fluorochromes being used. In the figure included here, I have attempted to explain this phenomenon and show how the optical filters of the microscope are set up to elimnate this. The emission and excitation spectra of some of the fluorochromes we commonly use are shown. FITC is shown in green, and 
is typical of fluorescein and its derivatives. This specific fluorochrome is the source of most problems associated with bleed through of signal to the TRITC spectra, shown in orange. This is due to the emission of energy by FITC at wavelengths overlapping the TRITC emission spectra, resulting in a false signal in the detection of the TRITC signal. The most effective way to eliminate this is by ensuring the use of the BP 585-615 filter, instead of the BP 550-615 filter, which covers more of the TRITC emission spectra but also includes more of the emission spectra from FITC. Additionally, the concentrations of the antibodies used can be adjusted to increase the TRITC signal and decrease the FITC signal, resulting in an increase in the ratio of desired signal to bleed through signal from FITC. The problem of bleed through and false signal is not as troublesome from the TRITC channel to the Far Red channel (Cy5, BODIPY 650, Alexa 633). The reason appears to be the detection width of the emission spectra for the Far Red channel (boxed area, Figure 1) compared to that of TRITC. This results in a greater ratio of desired signal to bleed through, but if the signal for TRITC is too great (as seen occasionally with rhodamine-phalloidin labeling) it may cause bleed through. This can be largely counteracted by decreasing the fluorescence intensity of the TRITC signal, which can be accomplished by decreasing the laser output for the laser which excites this fluorochrome (HeNe 543). Overall, when labeling more than one protein great care must be taken to optimize antibody concentrations before data can be retrieved with confidence, particularly when three labels are used. 


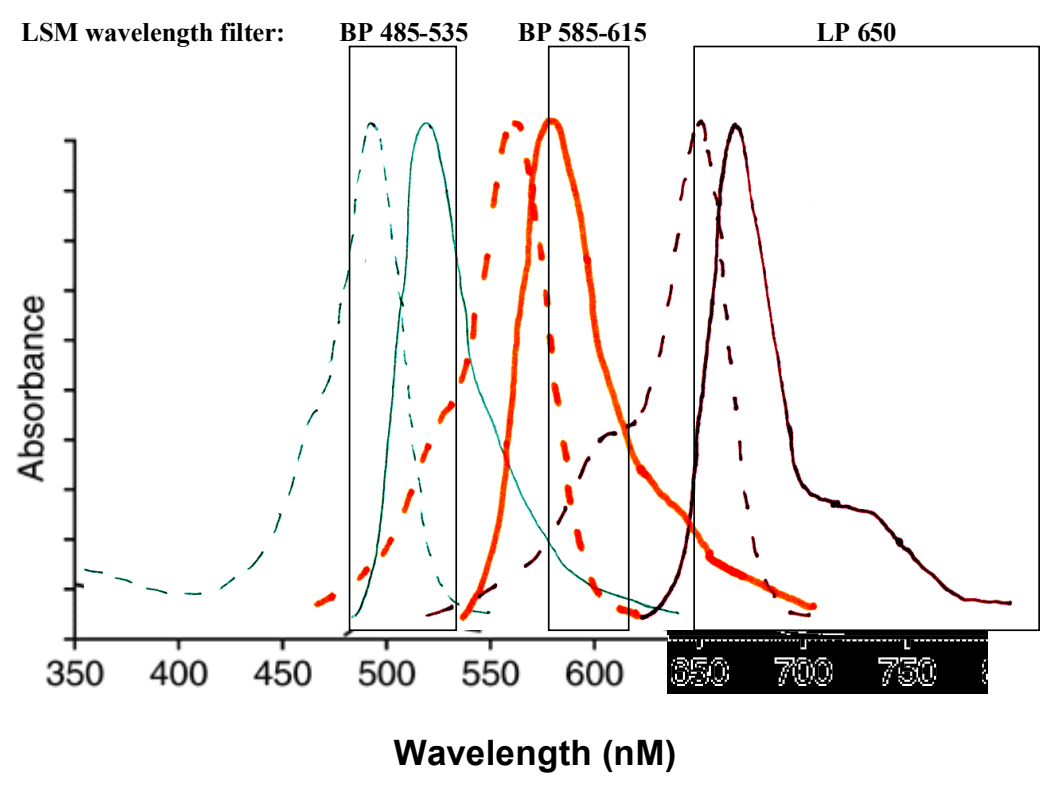

Figure 1 The use of filters which restrict the detection of light outside their designated wavelengths prevents the bleed through of signal from one fluorochrome to another. The emission spectra of the fluorochromes used are shown as solid lines (FITC shown as green, TRITC shown as orange, and Bodipy 650 shown as dark red) while their excitation spectra are shown as dotted lines. Note the filtersets designated (boxed regions) allows for the optimal detection of each fluorochrome's emission while excluding emission from the others.

The technique of gathering images is the most difficult to address in this format as it is a complex process with multiple variables. I will attempt to convey the tricks and techniques I have learned. In preface, the Zeiss LSM510 is a wonderfully cantankerous machine. Some days she will perform flawlessly and other days she will need restarted several times and still misbehave. The problems you will see include, but are not limited to: freeze-ups during image storing, multiple error messages, incompliance with commands and sluggishness. Sometimes it is best to simply return another day if multiple problems arise resulting in a major conflict between the user and the machine.

Upon placing the slide upon the stage, the first challenge is to locate cells looking through the eyepieces. This is sometimes more difficult than it should be. When labeling actin with either FITC or TRITC, the best way is to use that particular channel and focus up and down 
while moving the stage slightly. Look for a signal and try to focus on it. When using other labels which prove difficult to find by this method, it is helpful to use brightfield and first focus on the edge of the coverslip. Then move the stage to center the slide while searching for cells. Switch to fluorescence and if your labeling is adequate, the cells will be seen. One tip to remember is that even though the machine is labeled for it and the people running the facility will tell you otherwise, you cannot see the Far Red spectra through the eyepieces. It is nice however, upon laser scanning, which I'll discuss now.

After locating the cells they must be imaged by the computer to retain the data gathered. This process is daunting but simply follows the principle above relying on an adequate signal to noise ratio. Optimizing the signal to noise ratio in the image gathered, however depends solely on operator skill and experience. I will attempt to relay my experience here.

Setting up the microscope/computer to gather images involves turning on the correct lasers and implementing the correct settings/configurations for the fluorochromes used in the cell labeling. Understanding the excitation/emission spectra section above will direct the choices made here. Note that when using both FITC and TRITC, the BP 585-615 filter should always be used rather than the BP 550-615 filter, to minimize bleed through.

Once the correct configurations have been chosen the image must be gathered. Ideally this is a process which involves rapidly identifying the region to be scanned and focusing the image. The quicker this is done the better, as some fluorochromes bleach rapidly, causing a loss of signal. This also involves adjusting several settings to optimize the signal to noise ratio, as seen upon scanning. These settings include the pinhole, detector gain and the amplification gain. Adjustment of the amplification offset has proven of no help in adjusting image quality. The first setting to be considered is the pinhole. This is what gives the confocal microscope much of 
its resolving power by limiting the thickness of the area being focused on and imaged. For a further explanation of this concept see the Zeiss manual accompanying the machine. Adjusting the pinhole changes the thickness of the slice (optical slice) of sample being scanned. By adjusting this thickness, you can reduce background noise as well as noise from within the cell you are examining. These usually do not interfere with imaging, but when the signal is low or the cell is thick, they can be. The pinhole should be checked after setting the configurations and adjusted to the settings desired. For the $63 x$ lens, I generally start with a pinhole setting which gives an optical slice of around 2 microns. This is about the normal thickness of a cultured fibroblast and therefore most of the signal should be included. The pinhole must be set for each channel (signal from individual fluorochrome) used. After setting the pinhole, the gains need to be optimized for imaging. This is easily accomplished by using the "Find" button on the scan control panel. This quickly scans the image and automatically optimizes the signal to noise ratio. I find that adjusting the detector gain up after this is usually necessary for best resolution. Only the detector gain should be adjusted, unless the signal is weak. If this is the case and you are certain the cell is in focus, increasing the amplification gain may help slightly. At this point the resolution can be increased by adjusting the number of pixels being scanned as well as the time spent on each pixel. This is accomplished on the scan control panel, as well. Increasing the number of pixels being scanned increased image resolution and I use 1054x1054 pixels most often. There is a setting higher than this, but this gives a much larger files size with no appreciable increase in resolution. Adjusting the scan time also increases the resolution, and I usually use a scan time which gives an overall image scan time of 1 minute when finally scanning an image to save. Additionally, the number of times a pixel is scanned can be increased, resulting in a decrease in background noise. I most often use 2 for this setting. After 
changing these settings and scanning at the minimal time setting, if there is still poor image quality, there are a few things left to consider. If the image is bright but blurry or part of it is out of focus, the focus should be adjusted manually while continually scanning. If part of it remains out of focus, this part can be eliminated by adjusting the pinhole to reduce the optical slice. This is useful with cells that are thicker than average. I have found the lower limit of size on optical slice which still returns a quality image is around 0.5 microns, with a strong signal. If the image contains several structures which are desired, yet cannot be focused on simultaneously, the use of the $\mathrm{Z}$ stack function is very powerful. This involves reducing the optical slice and focusing to the upper and lower limits of the cell and marking these points with buttons in the $\mathrm{Z}$ stack control panel. Adjustment of the number of slices to be gathered (usually between 5 and 10) and implementation of the scan will return a series of scans representing individual planes from the cell. Unwanted slices can be eliminated and the wanted slices can be used to create a flattened image of all planes in focus with the 3D processing control panel. This can additionally be used to create 3D images of the cell, either as rotating views or stereoscopic images.

When dealing with imaging cells, different techniques are helpful. When examining slides with many of the desired cells to image, it is easy to simply start with the $63 \mathrm{x}$ objective and find the desired cells. These usually include labeling endogenous proteins or transfected Cos cells. However, when looking for the rare cell, such as in transfection of other fibroblasts, it is sometimes easier to start with a lower magnification, such as $20 \mathrm{x}$ and look for the desired cell. Upon finding and centering the cell, switch to $63 \mathrm{x}$ and add water, as this is a water immersion lens. 
Table 1- antibody concentrations used for immunofluorescence labeling. Antibody type is either monoclonal (m) or polyclonal (p).

Antibody concentrations are given as $\mathrm{mg} / \mathrm{ml}$.

\begin{tabular}{|l|l|l|}
\hline Cell Type/protein & Antibody (type) & Concentration or (dilution) \\
type & & \\
\hline Cos-1/endogenous & Phalloidin & $1: 1000-1: 250$ \\
\hline Cos-1/transfected & F1 & $1: 200$ \\
\hline & 4 C3 & $1: 2000$ \\
\hline C3H10T1/2, & Sigma anti-Flag & $1: 1000$ \\
\hline NIH3T3 & F1 endogenous & $1: 1000$ \\
\hline HS68 & & $1: 2000$ \\
\hline & FC3 & $1: 2000$ \\
\hline & Flag & $1: 1000$ \\
\hline & HA tag & $1: 500$ \\
\hline & Ptyr & $1: 100$ \\
\hline & & $1: 100$ \\
\hline
\end{tabular}


Appendix II

AFAP-110 clones generated 


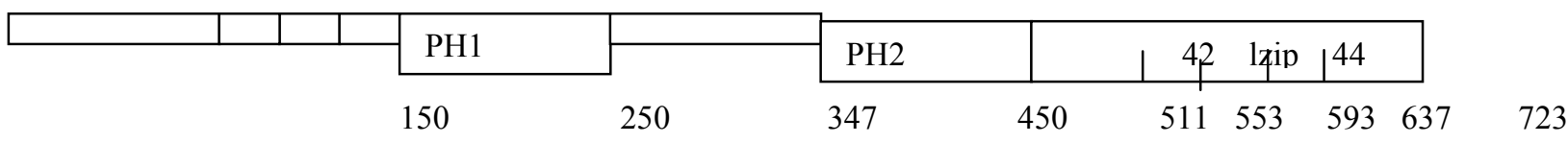

Mutants constructed by Joe B.

Vector-eGFP-c3 AFAP-110 cloned in by EcoRI from KS+(by Yong).

GFP-AFAP-110 $\Delta$ Spe- Van91I drop on from CMV-1AFAP-110 $\Delta$ Spe.

$\begin{array}{llll}\mathrm{Bp} & 558 & 697 \\ & 180 & 226\end{array}$

GFP-AFAP-110 $382-593$ SpeI-HindIII drop in from CMV-1 AFAP-110 $382-593$.

$\begin{array}{lll}\text { Bp } & 1161 & 1797\end{array}$

\begin{tabular}{lll}
\hline Residue & 381 & 594
\end{tabular}

GFP-AFAP-110 $\Delta$ Spe/Alzip Van91I drop on from CMV-1AFAP-110 $\Delta$ Spe into GFP-AFAP-110 llzip.

$\begin{array}{lllll}\mathrm{Bp} & 558 & 697 & \frac{1667}{1797} \\ & 180 & 226 & & 550594\end{array}$

GFP-AFAP-110 $\Delta$ Spe/ $\Delta 84-$ Van91I drop in of $\Delta$ Spe fragment into GFP-AFAP-110 $\Delta 84$.

$\begin{array}{llll}\mathrm{Bp} & 558 & 697 & 1667\end{array}$

$\begin{array}{llll} & 180 & 226 & 550\end{array}$

GFP-PH BglII fragment drop in from CMV-1-AFAP-110g462a/g757c into GFP in frame.

$\begin{array}{lll}\text { Bp } & 462 & 757\end{array}$

$\begin{array}{lll}\text { Residue } & 149 & 245\end{array}$

GFP-STK BglII fragment drop in from CMV-1-AFAP-110g757c into GFP in frame.

$\mathrm{Bp}$

$757 \quad 1161$

Residue

381

GFP-PHSTK BglII fragment drop in from CMV-1-AFAP-110g462a into GFP in frame.

$\begin{array}{lll}\text { Bp } & 462 & 1161\end{array}$

$\begin{array}{lll}\text { Residue } & 149\end{array}$

GFP-PHEND SpeI-HindIII fragment drop in from GFP-AFAP-110 into GFP-PH in frame.

Bp 462

Residue 149 


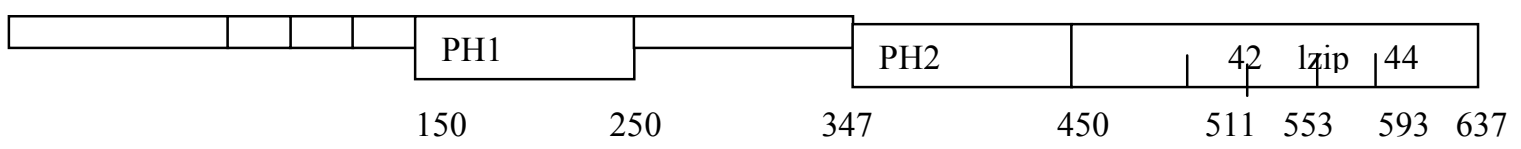

Mutants constructed by Joe B.

Vector-CMV-1 AFAP-110 cloned in by Kpn-Xba from KS+ (cloned in at EcoRI) with some KS+ MCS sequence.

AFAP-110 $\Delta$ Spe- created using site-directed mutagenesis.- same primers as for GEX-2T fusion protein, then Spe deletion.

Bp $558 \quad 697$

Residue $180 \quad 226$

AFAP-110 $4382-593$ simple Bgl II digestion.

$\begin{array}{lrr}\text { Bp } & 1161 & 1797\end{array}$

\begin{tabular}{lll}
\hline Residue & 381 & 594
\end{tabular}

AFAP-110 $\Delta$ Spe/ $\Delta 382-593-\mathrm{KpnI}-\mathrm{BglII}-\mathrm{XbaI}$ in wt AFAP-110-CMV-1.

\begin{tabular}{lllll}
$\mathrm{Bp}$ & 558 & 697 & 1161 & 1797 \\
\cline { 5 - 5 } & 180 & 226 & 381 & 594
\end{tabular}

AFAP-110 $\Delta$ Spe/ $\Delta$ lzip- ClaI drop in of $\Delta$ Spe fragment into AFAP-110 $\Delta$ lzip.

$\begin{array}{lcccc}\mathrm{Bp} & 558 & 697 & \frac{1667}{550594} & \\ & 180 & 226 & \end{array}$

AFAP-110 g462a Gives a new BglII site- mutagenesis

Bp $\quad$ g462a

Residue $\quad 148$

AFAP-110 g757c Gives a new BglII site- mutagenesis

Bp $\quad$ g757c

Residue 246

AFAP-110 g462a/g757c Gives 2 new BglII sites- Used KpnI-SpeI-BfrI three part ligation to join fragments from above.

Bp $\quad$ g757c

Residue 246 
AFAP-110 $\triangle$ PH- created by HindII-BglII-EcoRV in AFAP-110 g462a/g757c

$\begin{array}{lll}\mathrm{Bp} & 462 & 757\end{array}$

Residue $\quad 148 \quad 246$

AFAP-110 $\Delta$ STK- created by KpnI-BglII-ClaI in AFAP-110 g757c

Bp $\quad 757 \quad 1161$

Residue $\quad 246 \quad 382$

AFAP-110 $\Delta$ Spe/ $\Delta 84$ - ClaI drop in of $\Delta$ Spe fragment into AFAP-110 $\Delta 84$.

$\begin{array}{llll}\text { Bp } & 558 & 697 & 1667\end{array}$

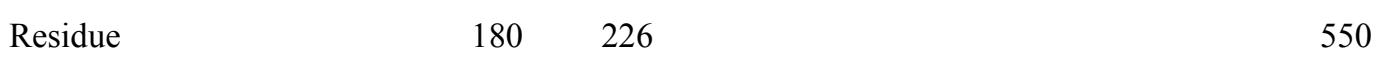

Additionally-

CMV-AFAP-110 $\Delta$ Spe with Src527F in CMV-1 via HindIII-XbaI drop in of spe into cmv-Src

CMV-AFAP-110 1 lzip with Src527F in CMV-1 via HindIII-XbaI drop in of spe into cmv-Src 
Appendix III

PH domain modeling 
During the course of the DNA/protein modeling course offered by the School of Pharmacy, I generated a model of the amino-terminal PH domain of AFAP-110. I chose to use homology modeling, as the structure was a rather large one, contained many regions with unpredictable tertiary structure, and several $\mathrm{PH}$ domain structures have been solved previously by NMR. As this PH domain demonstrated highest homology with the PH domain from $\beta$ Spectrin and this domain structure had been solved, this domain was used as a template. I used the modeling programs on the UNIX machines in the modeling laboratory to first align the sequences and then generate a preliminary structure. This structure was minimized for total energy and errors/incompatibilities were fixed by hand. This process was tedious and I found it to be limited in usefulness for the time and effort involved. Additionally, the final result after energy minimization was significantly different than other PH domain structures. This may have been due to errors in alignment, or problems at any of the other steps along the way.

In my search to overcome these problems, I began to use SwissPDBViewer, a software package found at the SwissProt site. This program allowed for the easy generation of a model of the amino-terminal $\mathrm{PH}$ domain based on the $\mathrm{PH}$ domain of $\beta$-Spectrin. Problems/incompatibilities were fixed easily and the resultant model appeared to follow the conserved structure of $\mathrm{PH}$ domains. This program also allowed for the generation of POV-Ray scenes of the model, giving high resolution images, as included here. 


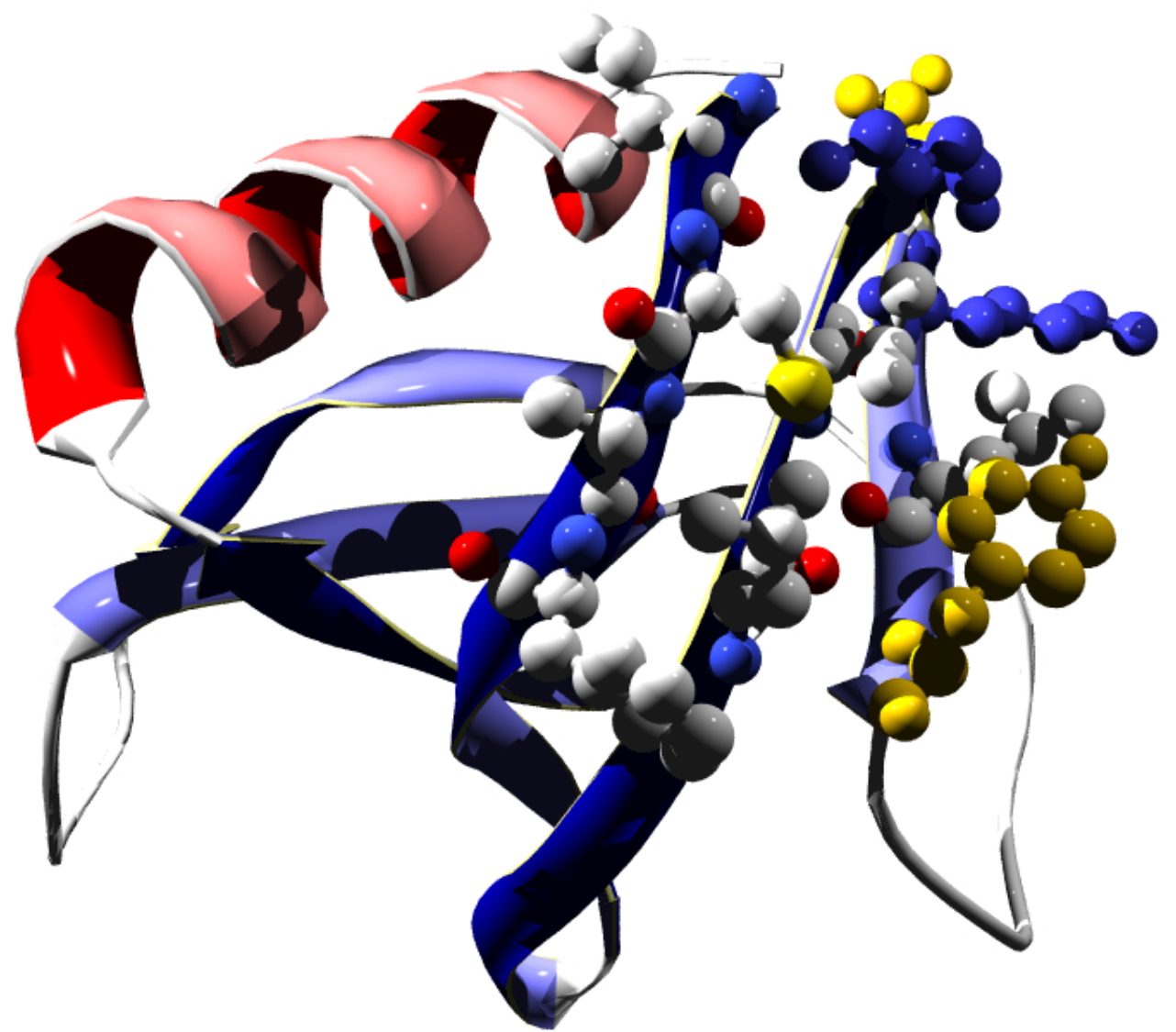

Figure 1 Model of the amino-terminal PH domain of AFAP-110. This model displays the backbone atoms as ribbon structures. Blue ribbons indicate beta strands, while red ribbons indicate the alpha helix. Ball and stick cartoons of the side-chain atoms of several residues thought to be involved in PKC binding are included, as well. These are color-coded: blue=basic, white $=$ hydrophobid, yellow=aromatic . 


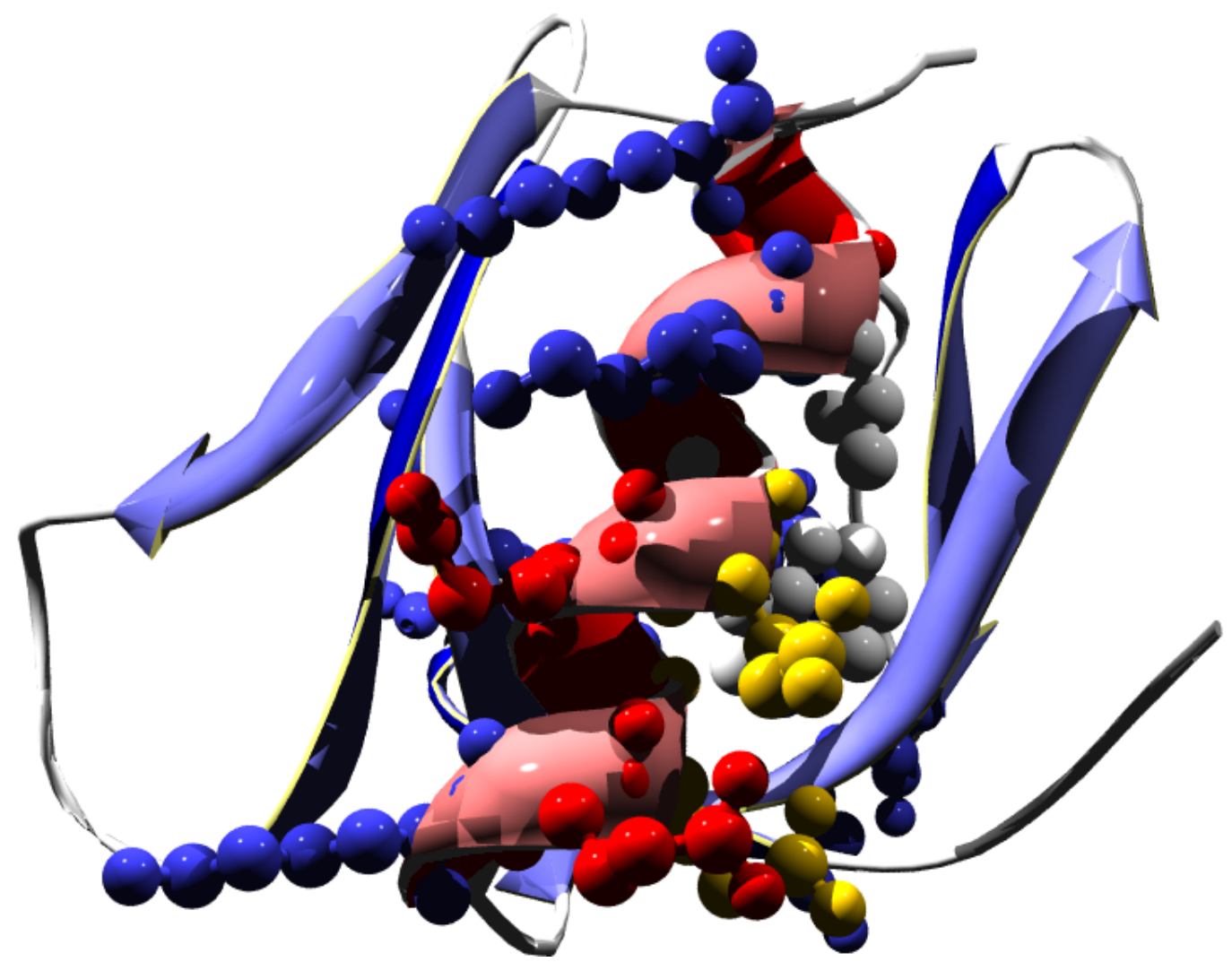

Figure 2 Model of the amino-terminal PH domain of AFAP-110. This is the same model shown in Figure 1, rotated to show the face thought to be involved in binding to WD40 repeatcontaining proteins, such as RACK1. Again, blue ribbons are beta strands and the red ribbon is the alpha helix. Side chain atoms are included which may be involved in RACK1 binding: blue= $=$ basic, red=acidic, white=aromatic, yellow $=$ hydrophobic. 


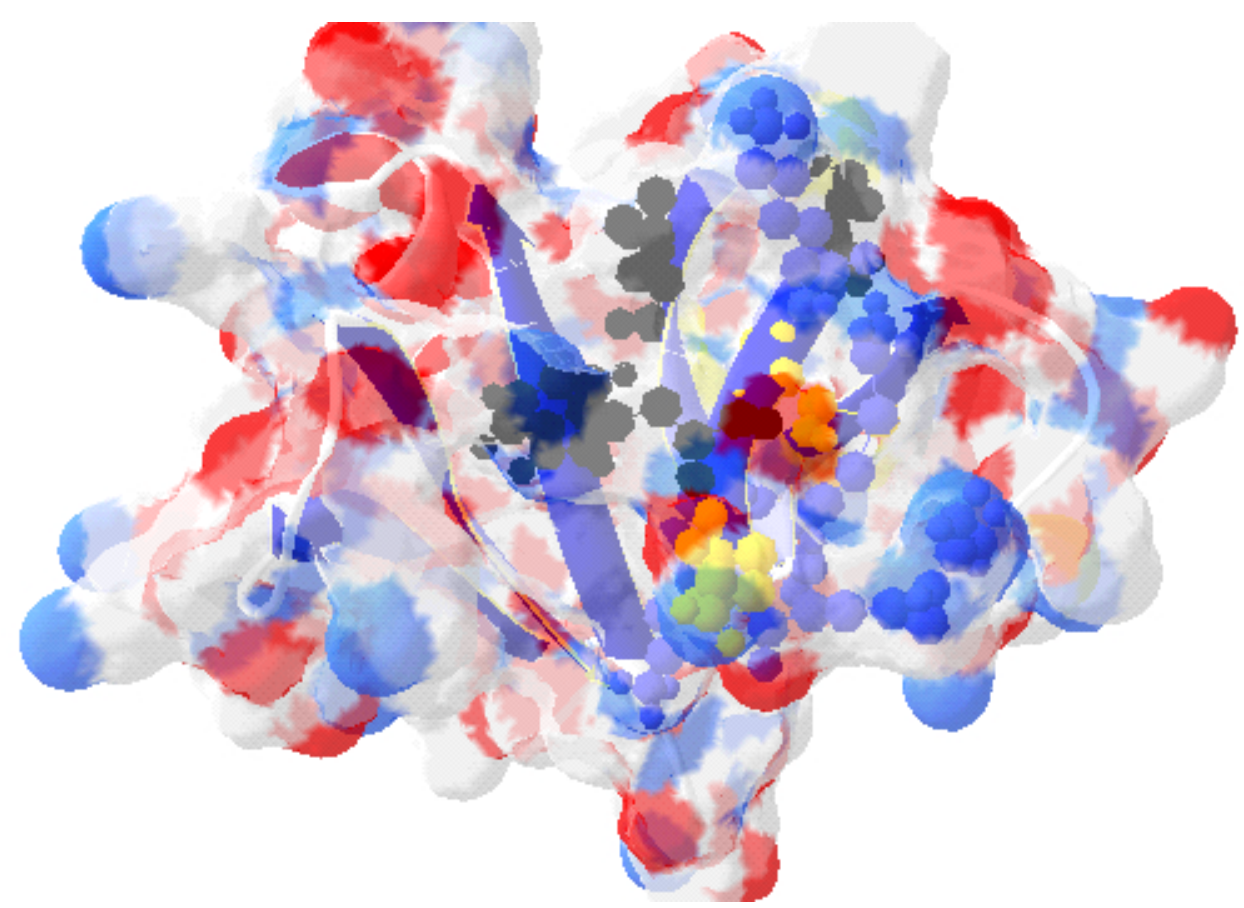

Figure 3 The model of the PH domain, in a view similar to that in Figure 1. This view includes the surface of the structure, calculated according to known atomic radii. This surface is partially transparent to allow the visualization of the cartoon beneath. The surface is color coded to display charge; blue is more negative and red is more positive. 


\section{Appendix IV}

AFAP-110 multimerizes via interactions between the PH domain and carboxyterminus in addition to leucine zipper-directed carboxy-terminal interactions. 
Previous reports have indicated that the carboxy-terminal region of AFAP-110 including the leucine zipper can direct the self-association of AFAP-110. Studies aimed at determining the region of AFAP-110 which binds this carboxy-terminus have revealed two separate sites capable of interacting with this region. As seen in figure 1A, the carboxy-terminus can interact directly with the carboxy-terminus of another molecule of AFAP-110 and with the amino-terminal PH domain of AFAP-110. Figure 1A includes western blot analysis of affinity absorptions of a GFP-tagged protein representing the carboxy-terminal 218 residues of AFAP-110 fused to GFP (GFP-Cterm). Fusion proteins representing either the amino-terminal PH domain (GST-PH) or residues 511-637 (GST-511-637), which includes the leucine zipper motif, were capable of affinity absorbing GFP-Cterm from Cos-1 cell lysate.

Mutants of the amino-terminal PH domain were expressed as GST-fusion proteins to determine the region responsible for interaction with the carboxy-terminus. Figure $1 \mathrm{~B}$ shows that deletion of residues 180-226 from this domain abrogates the affinity absorption of AFAP110 from Cos-1 cell lysate. Additional experiments have confirmed this result using purified recombinant AFAP-110 (data not shown). This figure additionally shows that either deletion of residues $226-240$ or mutation of residue 240 from tryptophan to alanine has no affect on the absorption of AFAP-110. In addition, a fusion protein representing the carboxy-terminal $\mathrm{PH}$ domain fails to affinity absorb AFAP-110. Thus the interaction between the amino-terminal PH domain and the carboxy-terminus depends on the integrity of residues 180-226 within the PH domain. 


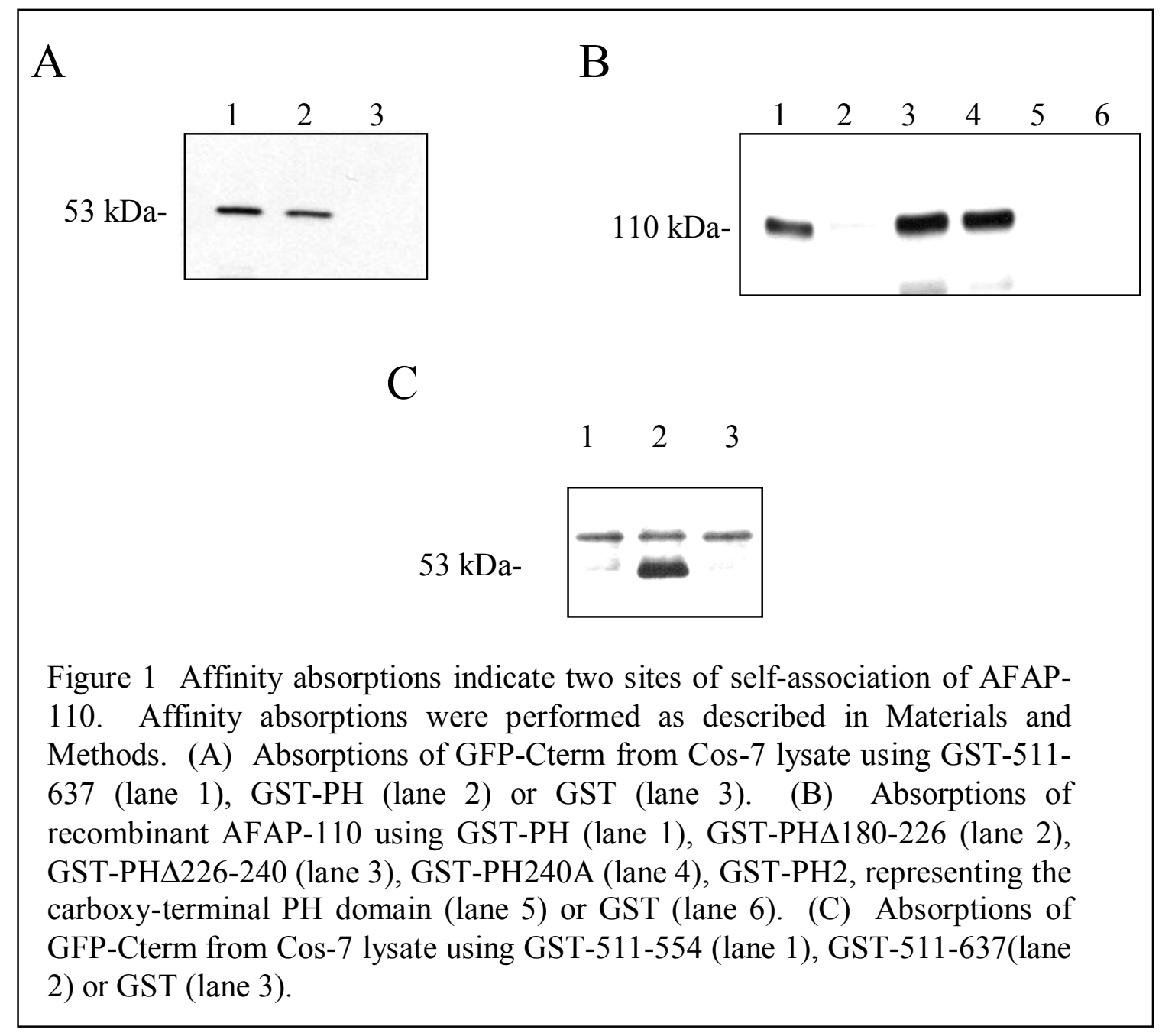

In order to determine the residues of the carboxy-terminus necessary for the homodimerization of this region, mutants of this region were used in affinity absorptions and are shown in Figure 1C. Deletion of the leucine zipper (residues 554-594) and adjacent carboxyterminal sequences (residues 595-637) abrogates the absorption of AFAP-110 from Cos-1 lysate (GST-511-554). Furthermore, deletion of the leucine zipper alone or residues 595-637 alone does not abrogate the absorption of AFAP-110 even though the leucine zipper alone is sufficient 
for absorption of AFAP-110 (data not shown). Thus the carboxy-terminal/carboxy-terminal interaction depends upon residues 554-637. These data indicate that the mechanism of selfassociation of AFAP-110 involves two sites of interaction.

PKC phosphorylation of AFAP-110 directs changes in self-association of AFAP-110

AFAP-110 was recently

A

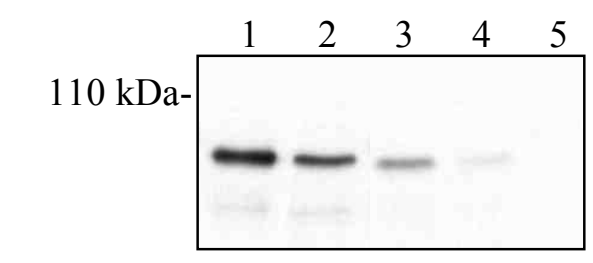

B

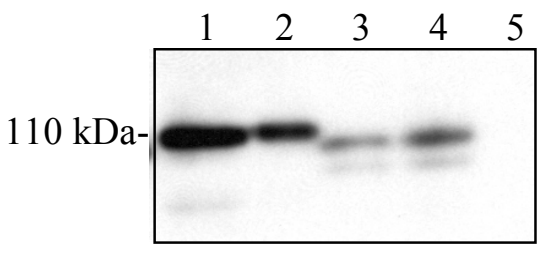

Figure 2 Affinity absorptions indicate PKC phosphorylationdependent self-association of AFAP-110. Affinity absorptions were performed as described in Materials and Methods. (A) Absorptions of recombinant AFAP-110 $0^{\Delta 176}$ using GST-511-637 as follows: AFAP-110 $10^{\Delta 176}$ (lane 3), and AFAP-110 1176 phosphorylated with $\mathrm{PKC}$, as described in Materials and Methods (lane 4). Affinity absorption of AFAP- $110^{\Delta 176}$ with GST is included (lane 5). Protein loading levels are shown from lysate as: AFAP-110 $10^{\Delta 176}$ (lane 1) and PKC phosphorylated AFAP-110 $10^{\Delta 176}$ (lane 2). (B) Absorptions from Cos-7 lysate using GST-511-637 as follows: AFAP-110 (lane 1, AFAP-110 co-expressed with Src527F (lane 2), AFAP-110 ${ }^{\Delta 176}$ (lane 3), and AFAP-110 1176 co-expressed with Src527F (lane 4). Affinity absorption of AFAP- $110^{\Delta 176}$ with GST is included (lane 5). found to be a PKC binding partner and substrate, as shown in Chapter 3. Additionally, the self-association of AFAP-110 was also shown to be altered in response to $\mathrm{PKC}$ activation downstream of Src activation. To determine if this is a direct consequence of $\mathrm{PKC}$ phosphorylation, recombinant purified AFAP-110 $10^{\Delta 176}$ was phosphorylated by $\mathrm{PKC}$ in vitro and subjected to affinity absorptions with the fusion proteins used above. This mutant, as described in Chapter 
1, lacks both the leucine zipper and adjacent, carboxy-terminal region. Therefore this mutant is likely unable to form interactions between carboxy-termini. The affinity absorption of this mutant with GST-511-637 therefore relies solely on interactions with the amino-terminal PH domain. Figure $2 \mathrm{~A}$ indicates that phosphorylation of recombinant purified AFAP- $110^{\Delta 176}$ by PKC reduces the ability of GST-511-637 to affinity absorb this mutant, compared to the nonphosphorylated mutant. Thus the interaction between the amino-terminal PH domain and the carboxy-terminal region is regulated by PKC phosphorylation. Affinity absorption of the same mutant from Cos-1 lysate supports this result, as this mutant undergoes a change in selfassociation when co-expressed with $\mathrm{Src}^{527 \mathrm{~F}}$ (data not shown). Additionally, the abrogation of the $\mathrm{PH}$ domain/carboxy terminal interaction reveals that the carboxy-terminal/carboxy-terminal interaction is not affected by $\mathrm{Src}^{527 \mathrm{~F}}$ co-expression. Figure $2 \mathrm{~B}$ shows affinity absorptions from lysate of Cos-1 cells expressing AFAP-110 $0^{\Delta 180-226}$, the mutant deficient for PH domain/carboxyterminal interaction. Absorptions with GST-511-637 therefore rely on carboxyterminal/carboxy-terminal interactions. In this instance, AFAP- $110^{\Delta 180-226}$ co-expressed with $\mathrm{Src}^{527 \mathrm{~F}}$ is affinity absorbed with a similar efficiency as this mutant expressed alone. Both appear to be absorbed with a similar efficiency as wild-type AFAP-110 co-expressed with Src. This is consistent with the hypothesis that AFAP- $110^{\Delta 180-226}$ is reduced to a dimeric state by virtue of the abrogated PH domain/carboxy-terminal interaction. Together, these results indicate the PH domain/carboxy-terminal interactions are regulated by cellular signals, in contrast to carboxyterminal/carboxy-terminal interactions.

PKC phosphorylation of the PH domain and adjacent region alter the affinity of these fusion proteins for AFAP-110 
As PKC phosphorylation altered the $\mathrm{PH}$ domain/carboxy-terminal interaction but failed to alter the interaction between carboxy-termini, it was surmised that phosphorylation of the $\mathrm{PH}$ domain or surrounding region may be responsible for this effect. Indeed, this amino-terminal PH domain contains two serine residues which fit the PKC consensus phosphorylation sequence. Furthermore, the adjacent carboxy-terminal region contains multiple sites which also fit this consensus and has thus been termed the serine/threonine kinase interaction (STK) domain. Fusion proteins were used to examine the effects of PKC phosphorylation of this region on $\mathrm{PH}$ domain/AFAP-110 interactions. GST-PH, which represents the amino-terminal PH domain, and GST-PHSTK, which represents this PH domain and adjacent carboxy-terminal region (residues 248-347) were phosphorylated in vitro with recombinant PKC $\alpha$. These phosphorylated fusion proteins were used to affinity absorb recombinant purified AFAP-110 and were compared to affinity absorptions

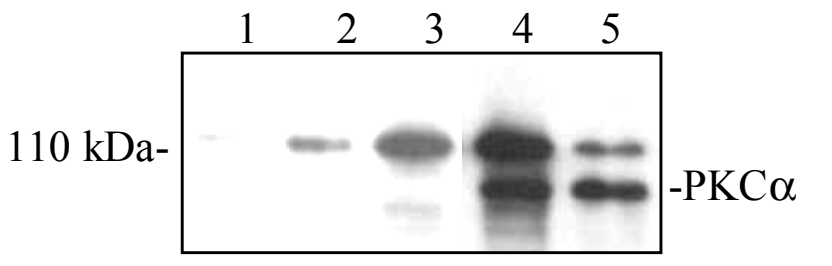

Figure 3 PKC phosphorylation of PH domain-containing fusion proteins alter their affinity for AFAP-110. Affinity absorptions and $\mathrm{PKC}$ phosphorylations were performed as described in Materials and Methods. (A) Absorptions of recombinant purified AFAP-110 were performed using the following: GST (lane 1), GST-PH (lane 2), GST-PHSTK (lane 3), PKC phosphorylated GST-PH (lane 4), PKC phosphorylated GSTPHSTK (lane 5). mAb 4C3 revealed the presence of AFAP110 , and subsequent reprobing with anti-PKC $\alpha$ revealed the presence of recombinant $\mathrm{PKC} \alpha$.

\section{affinity absorptions} with nonphosphorylated forms of the same fusion proteins. The results are seen in Figure 3. PKC phosphorylation appears to have opposite effects on the abilities of 
these proteins to affinity absorb AFAP-110. GST-PH appears to have an increased affinity for AFAP-110 upon phosphorylation, while GST-PHSTK has a decreased affinity for AFAP-110 upon phosphorylation. One possible explanation for this result is that interactions between the $\mathrm{PH}$ domain and the STK region may occur and display an increased affinity upon PKC phosphorylation. This may exclude the carboxy-terminal region, which would result in a change in self-association similar to that seen here. Additionally, this would also predict that the phosphorylated PH domain alone would exhibit an increased affinity for AFAP-110, as seen here. This would presumably occur by virtue of interactions between this phosphorylated GSTPH fusion protein with the STK region of the AFAP-110 molecule being affinity absorbed.

Possible other explanations for this result include competition for binding sites with PKC, which was included in this assay to phosphorylate the fusion proteins. To examine this possibility, the membrane was reprobed with antibodies to visualize PKC in the absorbate. The results indicated that equal amounts of PKC were present in both GST-PH and GST-PHSTK absorbates (Figure 3). Thus, it seems unlikely that PKC would be competing with AFAP-110 for affinity absorption in one but not both of these.

Future work on the self-association of AFAP-110 needs to address several issues. Firstly, the results presented here need to be recapitulated to confirm them (Figures 2 and 3, specifically). To further address the idea that $\mathrm{PH} /$ carboxy-terminal interactions are regulated by PKC phosphorylation and carboxy-terminal/carboxy-terminal interactions are not, an experiment using PKC to phosphorylate GST-PH and GST-PHSTK should also include GST-511-637. The idea that the PH domain and STK region interact needs to be examined. Two attempts have been made to affinity absorb GFP-STK, representing the STK region fused to GFP, with GST-PH. The results indicated GFP-STK was absorbed. This result should be confirmed, including the 
$\mathrm{PH}$ domain mutants to ask which portion of the $\mathrm{PH}$ domain contacts the STK region. Additionally, the GST-PH and GST-PHSTK fusion proteins could be phosphorylated by PKC in vitro and subjected to tryptic analysis to determine if the resultant radioactive spots correspond to those seen in wild-type AFAP-110 upon phosphorylation by PKC. As one or more of the potential sites in the STK region are likely phosphorylated, experiments with the mutant form of AFAP-110 lacking this region may aid in these studies. Preliminary results with this mutant indicate deletion of this region may block the ability of PKC to induce the change in selfassociation as well as cytoskeletal reorganizations. These results should be confirmed. 


\section{Appendix V}

The amino-terminal PH domain of AFAP-110 may direct interactions with RACK1 
PH domains have been shown to interact with proteins which contain WD40 repeats, such as G $\beta \gamma$ and RACK1. RACK1 is the receptor for activated C kinase, which has been shown recently to interact with Src in addition to $\mathrm{PKC}$, as described in the discussion section. Both $\mathrm{PH}$ domains from AFAP-110 share homology with $\mathrm{PH}$ domains which bind these proteins within the portion of the PH domain thought to coordinate this interaction. Table 1 demonstrates this homology, and presents a consensus for this binding. As in the case of PH/PKC interactions as detailed in the introduction, this consensus also indicates the conserved hydrophobic residues thought to stabilize the structure, as identified from solved PH domain structures.

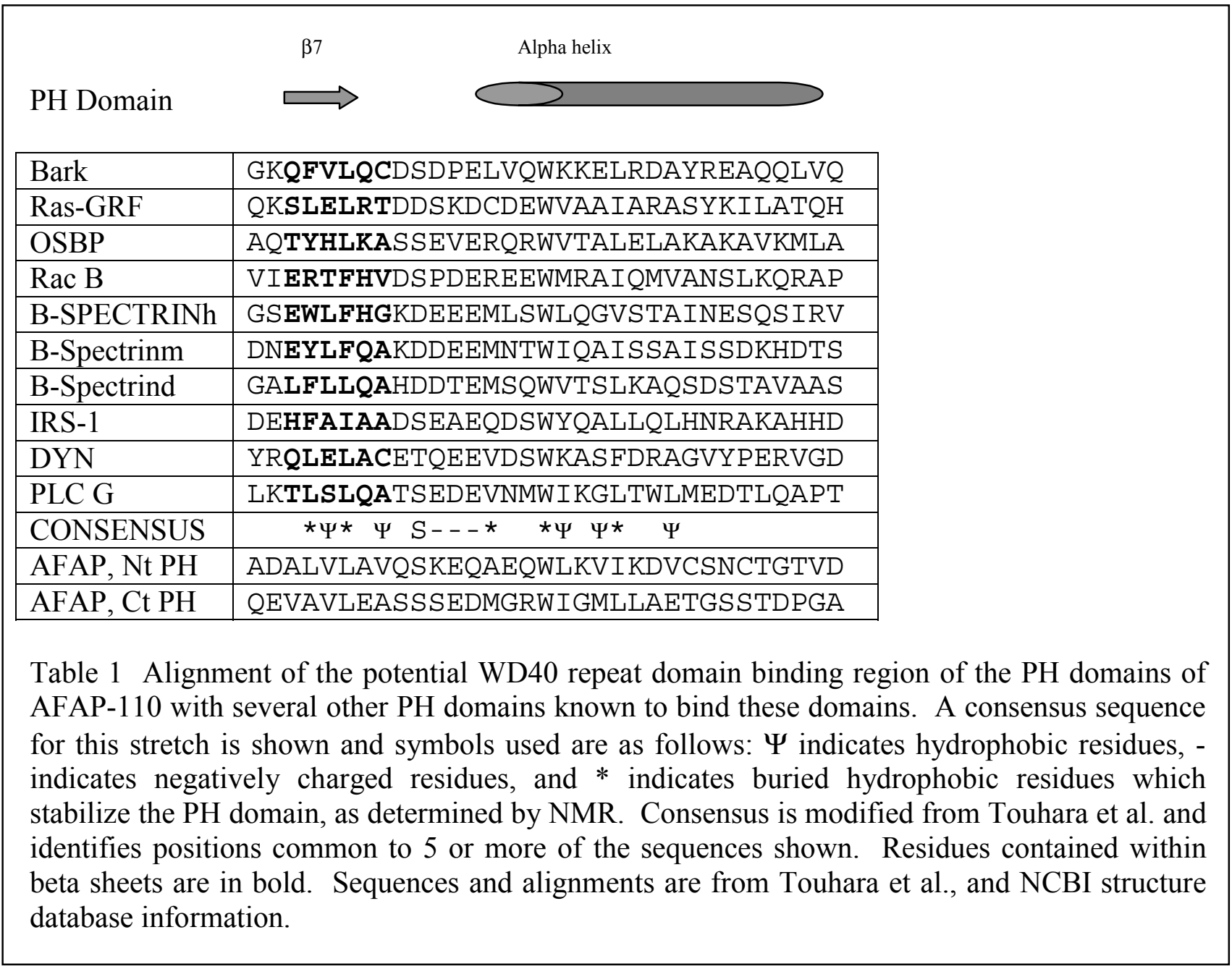


To test the potential interaction between the PH domains of AFAP-110 and RACK1, affinity absorptions with these domains were performed. Figure 1 includes these absorptions, which indicate the amino-terminal PH domain can affinity absorb RACK1 from chick brain lysate. The carboxy-terminal PH domain failed to absorb RACK1. This experiment was repeated, and indicated identical results. Co-immunoprecipitations using anti-RACK1 were performed, and SDS-PAGE/western blot analysis indicated that AFAP-110 was coimmunoprecipitated, shown in Figure 1B. This blot was stripped and reprobed and indicated that $\mathrm{PKC}$-reactive bands were absent, using a pan-PKC antibody. This is in agreement with previous reports, which have indicated an inability to co-immunoprecipitate PKC using RACK1 antibodies. Additionally, Figure 1B shows that the interaction between AFAP-110 and RACK1 appears to be unaffected by treatment with 100nM PMA for 1 hour. This experiment was performed, however, in NIH3T3 fibroblasts, which appear to be unresponsive to this treatment in respect toward cytoskeletal rearrangements. These results indicate a potential interaction between AFAP-110 and RACK1, a protein involved in the regulation of both Src and PKC.

A

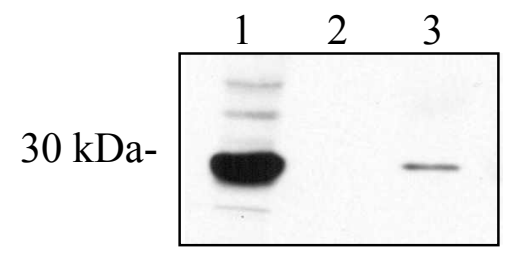

B

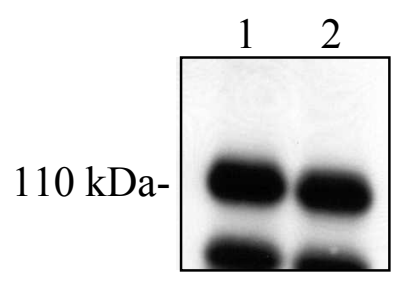

Figure 1 AFAP-110 may interact with RACK1 through the amino-terminal PH domain. (A) Absorptions of RACK1 from chick brain lysate using GST (lane 2) or GST-PH (lane 3). Lane 1 was loaded with $5 \mu \mathrm{g}$ lysate. (B) Coimmunoprecipitations of AFAP-110, as visualized with $\mathrm{mAb} 4 \mathrm{C} 3$, with antiRACK1 antibodies. Immunoprecipitation was from NIH3T3 cells (lane 1) and NIH3T3 cells treated with 100nM PMA for 1 hour (lane 2). 
Appendix VI

AFAP-110 localization in epithelial cells 
To examine the localization of AFAP-110 in epithelial cells, Caco-2, MDCK, and A431 cells were examined. The results shown here indicate that AFAP-110 is colocalized with actin filaments inside Caco-2 cells which are confluent, but fails to colocalize with actin at cell/cell junctions. These junctions are thought to maintain the integrity of the epithelial sheet and prevent cell migration from the sheet. AFAP-110 is found in actin-rich structures at the cell membrane in cells which are not touching other cells or in portions of cells which are not abutting other cells, as seen in Figure 1. Similar results were seen in A431 cells and MDCK cells. Additionally, treatment of confluent cells with $100 \mathrm{nM}$ PMA for 1 hour to activate PKC results in the detachment of the cells from one another and AFAP-110 is found in actin-rich membrane structures (data not shown). This indicates AFAP-110 may be involved in the destabilization of cell/cell junctions which are necessary for epithelial cell migration. Transfection experiments with GFP-tagged forms of AFAP-110 supports this. GFP-AFAP-110 expressed in Caco-2 cells, shown in Figure 2, colocalizes with actin filaments within these cells, but fails to colocalize with actin structures at cell/cell junctions. In contrast, GFP-AFAP-110 ${ }^{\Delta z i p}$ is found in actin-rich structures at the cell membrane. As this mutant activates cSrc, it seems likely that AFAP-110 could be involved in the regulation of cell/cell junctions. 


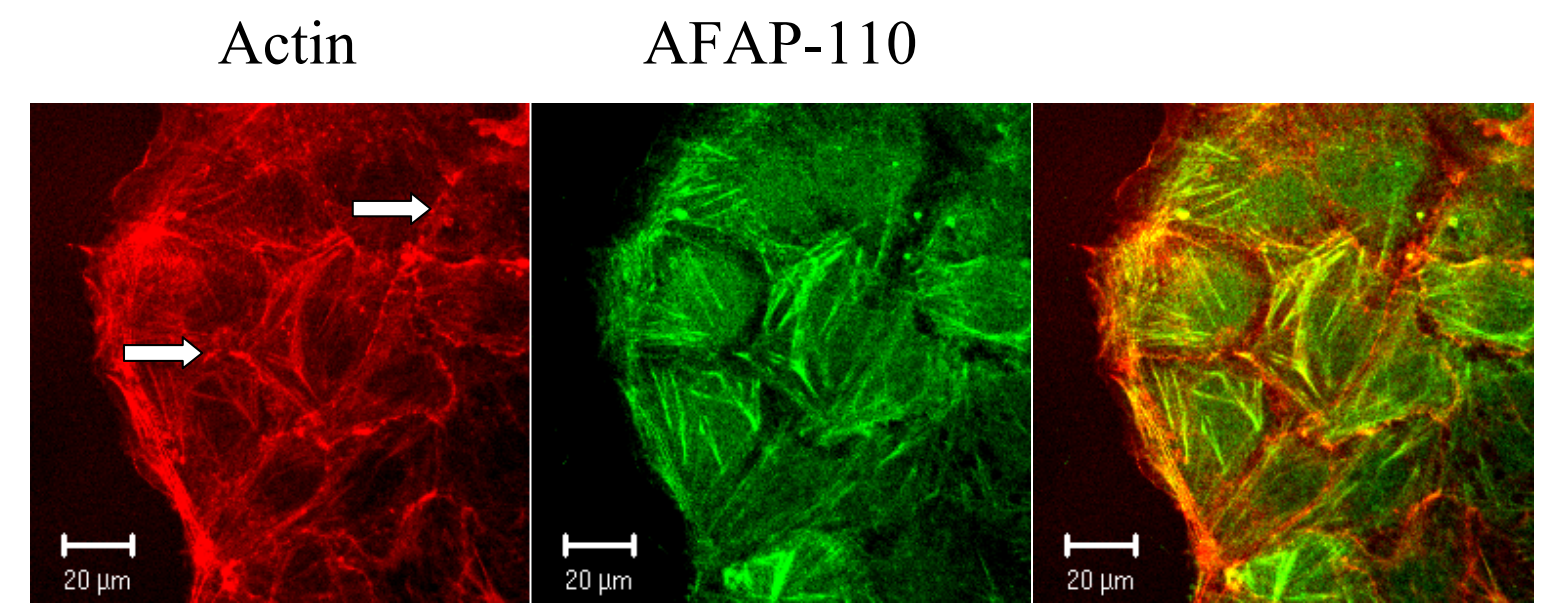

Figure 1 AFAP-110 is absent from cell/cell junctions in Caco-2 epithelial cells. Caco-2 cells were fixed and stained with BODIPYPhalloidin to visualize actin and ab F1 to visualize endogenous AFAP-110. Actin at cell/cell junctions are highlighted with arrows. 
Actin

GFP-AFAP-110

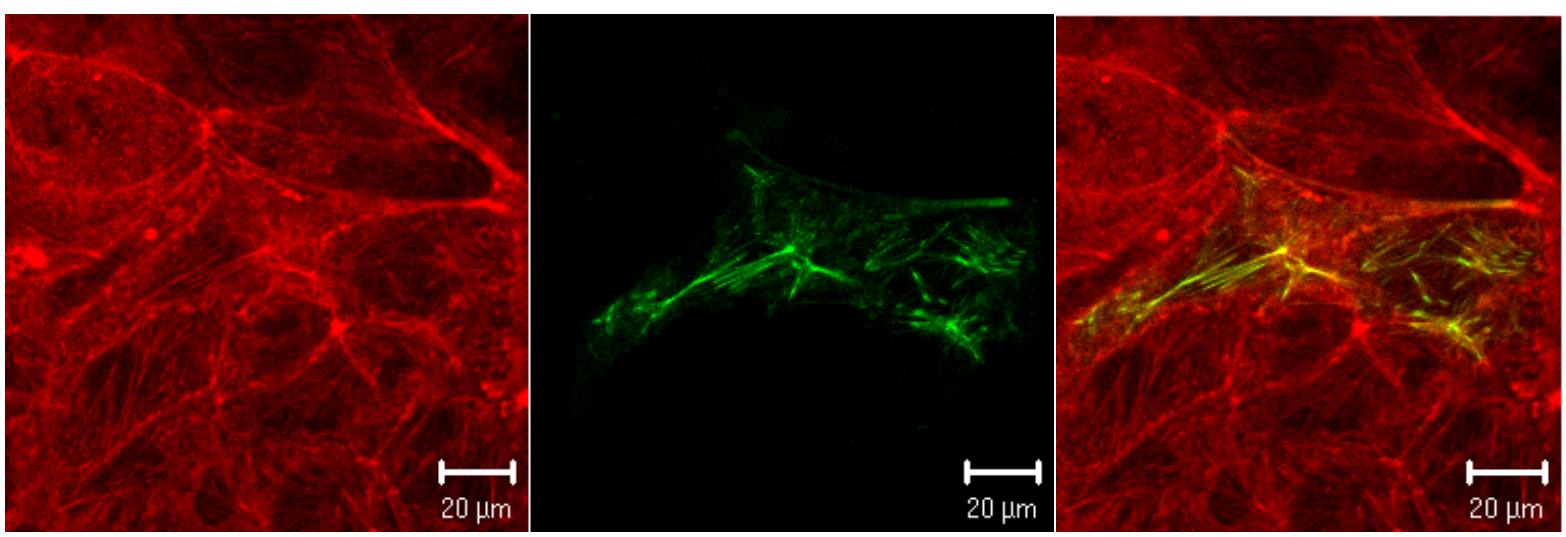

Actin

GFP-AFAP-110 $10^{\Delta \text { zip }}$
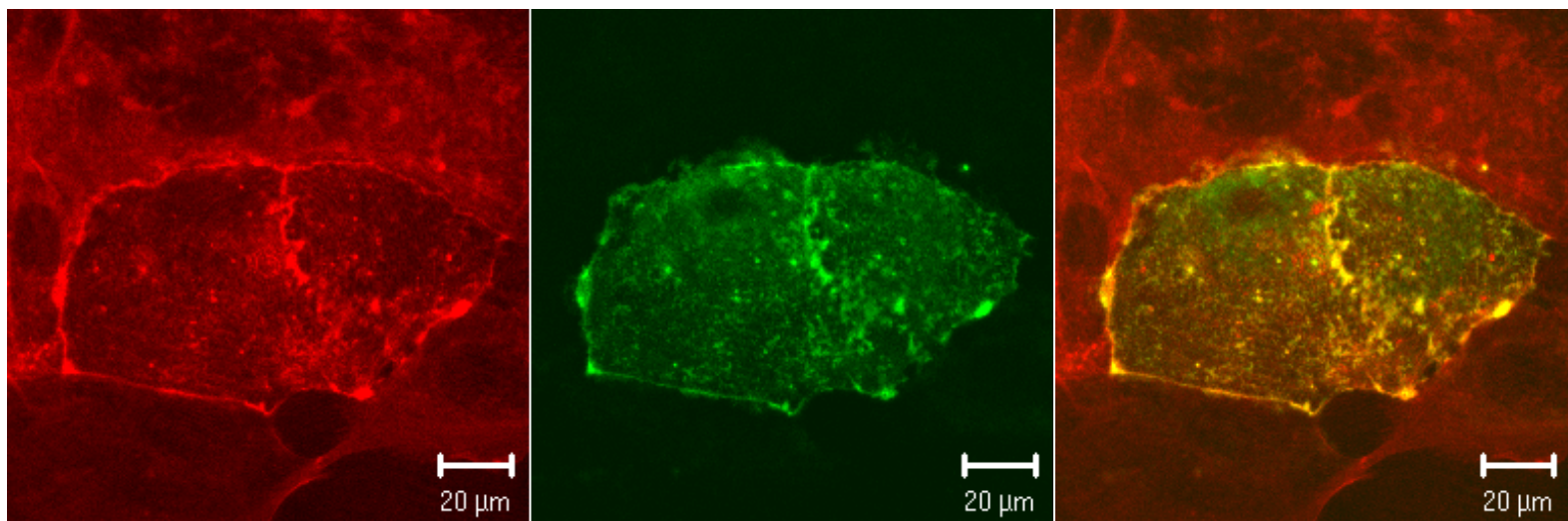

Figure 2 AFAP-110 $\Delta$ lzip is localized at the cell membrane in Caco-2 cells. Caco-2 cells were transfected using Effectene (Qiagen). Cells were grown to confluence and actin was visualized with BODIPY-Phalloidin. 


\section{Curriculum Vitae}

Joseph M. Baisden

\section{Personal}

Born: 12/16/1972 Winchester, VA

Married, two children (wife Jamie, children Kailani and Matthew)

US citizen

Home: 424 Stewart St. Morgantown, WV 26505

Work: 2822 MBR Cancer Center, West Virginia University, Morgantown, WV

26506-9300

\section{Education}

1991-1995 - B.A. Biology, West Virginia University, Morgantown

2001 - Ph. D. Microbiology, Immunology and Cell Biology

1995-2003 (expected) - M.D., West Virginia University

Dissertation: AFAP-110 is a cSrc activator

Defended 6/27/01

Advisor, Dr. Daniel C. Flynn

(Dept. of Microbiology, Immunology and Cell Biology, MBR Cancer Center, West

Virginia University, Morgantown, WV)

\section{Professional Experience}

1993-1995: Undergraduate research in the lab of Dr. Daniel C. Flynn, WVU

1997: Teaching assistant, WVU

1997-2001: Graduate research assistant in the lab of Dr. Daniel C. Flynn, WVU

\section{Society Membership}

1. American Society for Cell Biology (ASCB): Student member

2. American Association for Cancer Research (AACR): Student member

3. American Medical Association (AMA): Student member 


\section{Honors}

1. Presidential Scholarship, West Virginia University. 1991-1995.

2. Beta Beta Beta honor society, 1993-1995.

3. Golden Key, College of Arts and Sciences honor society, 1993-1995.

4. Phi Beta Kappa, honor society, 1995.

5. Graduated summa cum laude, 1995.

6. Runner-up, best poster, OSU Medical Scientist Student Forum, 1997.

7. Keystone Symposium Student Travel Award, 2000.

\section{Laboratory Techniques:}

Cell Culture \& Experimentation

Cell culture

Radio- and immuno-labeling

Protein Biochemistry

PAGE, Western blot analysis

Immunoprecipitation

Fusion protein production/purification

Phosphotryptic and phosphoamino acid analyses

in vitro phosphorylation

DNA manipulation

Agarose and acrylamide gel electrophoresis

DNA restriction digestions and sub-cloning

Small and large scale DNA preparation

Site-directed mutagenesis

PCR

Microscopy

Confocal microscopy (laser scanning)

Conventional light microscopy

\section{Abstracts}

J.M. Baisden, and D.C. Flynn. AFAP-110 is a PKC substrate with the potential to rearrange the actin cytoskeleton. Keystone Symposium, 2000. Taos, NM.

J.M. Baisden, H.G. Zot, and D.C. Flynn. Role of AFAP-110 as a cytoskeletal effector of cell signals. American Association for Cancer Research, 1999. Philadelphia, PA.

J.M. Baisden, Y. Qian, H.G. Zot, and D.C. Flynn. AFAP-110 can induce changes in actin filament integrity and membrane ruffling in response to signals involving Src. American Society for Cell Biology, 1998. San Francisco, CA. 
J.M. Baisden, D.C. Flynn, S.G. Graber, and K. Dupree. Role of the Pleckstrin Homology domain of actin filament associated protein $110 \mathrm{kDa}$. The Medical Scientist Student Forum, 1997. Columbus, OH.

Guappone, A.C., Baisden, J.M., Bellotte, J.B., Koay, T.C., Gibb, T., Flynn, D.C. A mechanistic study of SH2/SH3 stable complex formation between pp60src and AFAP-110. American Association for Cancer Research, 1995. Toronto, Canada. Awarded outstanding poster.

\section{Publications}

1. Qian Y, Baisden JM, Cherezova L, Summy JM, Guappone-Koay A, Shi X, Mast T, Pustula J, Zot HG, Mazloum N, Lee MY, Flynn DC. PKC Phosphorylation Increases the Ability of AFAP-110 to Cross-link Actin Filaments. Mol Biol Cell. 2002 Jul;13(7):231122.

2. Baisden JM, Gatesman AS, Cherezova L, Jiang BH, Flynn DC. The intrinsic ability of AFAP-110 to alter actin filament integrity is linked with its ability to also activate cellular tyrosine kinases. Oncogene. 2001 Oct 4;20(45):6607-16.

3. Baisden JM, Qian Y, Zot HM, Flynn DC. The actin filament-associated protein AFAP110 is an adaptor protein that modulates changes in actin filament integrity. Oncogene. 2001 Oct 1;20(44):6435-47. Review.

4. *Qian Y, *Baisden JM, Zot HG, Van Winkle WB, Flynn DC. The carboxy terminus of AFAP-110 modulates direct interactions with actin filaments and regulates its ability to alter actin filament integrity and induce lamellipodia formation. Exp Cell Res. $2000 \mathrm{Feb}$ 25;255(1):102-13.

5. Qian Y, Guappone AC, Baisden JM, Hill MW, Summy JM, Flynn DC. Monoclonal antibodies directed against AFAP-110 recognize species-specific and conserved epitopes. Hybridoma. 1999 Apr;18(2):167-75.

6. Qian Y, Baisden JM, Westin EH, Guappone AC, Koay TC, Flynn DC. Src can regulate carboxy terminal interactions with AFAP-110, which influence self-association, cell localization and actin filament integrity. Oncogene. 1998 Apr 30;16(17):2185-95.

* Co-first authors.

\section{Manuscripts in preparation}

- Baisden JM, Gatesman AS, Summy JM, Liu M, Flynn DC. AFAP-110 activates Src through SH3 interactions in response to PKC phosphorylation.

- Baisden JM, Gatesman AS, Cherezova L, Guappone AC, Flynn DC. Multimerization of AFAP-110 is regulated by PKC phosphorylation. 


\section{References}

Dr. Daniel C. Flynn, PhD

Scientific Director, Mary Babb Randolph Cancer Center

Associate Professor, Department of Microbiology/Immunology/Cell Biology

West Virginia University School of Medicine

PO Box 9300

Morgantown, WV 26506

304-293-6966

dflynn@hsc.wvu.edu

Dr. Michael E. Galloway, MD

Assistant Professor

Radiology, Section of Radiation Oncology

PO Box 9235

Morgantown, WV 26506

mgalloway@hsc.wvu.edu

Dr. John B. Barnett, PhD

Chairman, Department of Microbiology/Immunology

West Virginia University School of Medicine

PO Box 9177 Health Sciences Center North

Morgantown WV 26506

304-293-2649

jbarnett@hsc.wvu.edu 